\begin{abstract}
A complete relativity in all phenomena, with relative scale invariance in physical laws, is postulated and discussed.

Postulates are followed with definitions of new terms which may be used in the current and follow-up papers written in the context of the theory. In order to conform to this physics, a redefinition and generalization of some terms and factors already in use has also been presented.

This is followed by hypotheses on nature and mechanics of natural phenomena, based on the postulates of the theory, along with suggestions on the formulation of scale invariant physics.

In conclusion, the theory suggests that everything must be completely relative in order to exist and everything must evolve in order to conserve this relativity.
\end{abstract}




\title{
Complete Relativity: Nature of observables
}

\author{
Mario Ljubičić (Amenoum) \\ 108. brigade ZNG 43, 35252 Sibinj, Croatia (amenoum.org) \\ mljubicic99@gmail.com
}

December 23, 2022

\section{Introduction}

A theory of everything should provide a framework which can be used to qualitatively describe all phenomena, without exceptions - special cases. Complete relativity with incorporated invariance to scale has such power, opening a path to profound insights into fundamental mechanics of natural phenomena.

There's nothing abstract about physical reality. Therefore, the best approach to understand nature is to combine and balance the power of mathematical language with experience and observation on all scales of energy and in the process approach problems from different perspectives, rather than limit interpretation to that of a mathematician, physicist or a biologist.

Increasing complexity of mathematical language, for example, might make equations more elegant but beyond certain point it becomes harder to understand their correlation with physical processes, as elegance is neither abstract nor absolute in reality. Specialization, combined with religion, will create illusion. Illusion of understanding and illusion of knowledge.

Here, the aim is simply to understand nature as much as possible. The theory is the result of research, experience, observation, computation and logic that followed strong intuition suggesting that non-intuitive abstract and absolute reality is just an interpretation chosen, consciously or unconsciously, by a biased observer and it cannot be absolutely correct.

This, and follow-up papers, will provide foundations and evidence for the theory.

\section{Postulates}

Here are the postulates of Complete Relativity (CR). They are all entangled and one may stem from the other, but not always in apparent way. 


\subsection{Everything is relative (everything is variable)}

$$
\Delta E<>0
$$

No system can be completely isolated. Everything existing must be absorbing and radiating energy at some scale all the time, but never in the same amount during the same instant of time as that would make it absolutely unchanged (identical) or invariant to time.

Maintenance of relative constancy requires energy.

$$
\Delta E \neq \infty
$$

There is no single, absolute and infinite universe (Universe). For any observer, there exists a finite number of universes with mutually entangled characteristics, exchanging energy between each other.

One could argue that the sum of all universes is infinite and that sum is a single absolute entity. However, such Universe is absolutely unobservable. In the context of $\mathrm{CR}$, all existence has to be relative and thus observable at some scale.

Infinities and zeros as absolute values are mathematical abstractions - in reality they must be relative, as any other value.

Any physical entity having absolute properties would have to be absolutely isolated in order for these properties to remain constant. With no ability to change (exchange energy) such absolutely elementary entities would be unobservable. Thus, relativity is an intrinsic property of reality.

Relativity requires energy of different scales and, for each scale, a reference frame will exist from which that energy forms quanta of a medium for finite speed of information (energy) transfer.

Any distinct form of energy may be considered as an universe, however, in this context, universe will generally represent a particular scale of energy.

As transfer and transformation of energy require capacity for energy storage, structural quanta of any medium must have real size.

- Every universe must be divisible - contained within a larger universe and contain universes of smaller scale,

- reality containing these universes must be at least 3-dimensional,

- universes of different magnitude (vertically parallel universes) are selfsimilar but instances are evolutionary dominantly separated in time, 
- universes of the same magnitude (horizontally parallel universes) are similar but instances are evolutionary dominantly separated in space.

Since existence requires continuous exchange of energy, any relative constants (energies) must be oscillations in some reference frames. None of these oscillations can be absolutely stable - even the oscillation itself must change relative to something. Thus, each form of existence (energy) must evolve, either progressively or regressively. Since energy cannot occupy space of absolute 0 size (length, width, height), reality requires three dimensions.

Universes cannot have more than 3 spatial dimensions (there is no space for them!), however, due to various scales of energy, space can and will be effectively divided into subspaces which may be relatively isolated but correlated with other scales. In that case, multidimensional manifolds may be used to describe reality. But one should be careful not to declare such entanglements (couplings) intrinsic and absolutely constant properties of reality - the strength of any entanglement must be variable in completely relative reality.

In $\mathrm{CR}$, the scale of a universe generally represents a discrete vertical energy level, analogous to horizontal energy levels in Quantum Mechanics (QM).

Relative to difference in scale between observer energy and energy of observables, phenomena may be interpreted as physical (real) or mental (imaginary), however, every mental phenomena must have a physical interpretation at some scale, and vice versa.

Due to required evolution (oscillation), energy in a universe is constantly transferred and transformed. Due to finite speed of transfer and transformation, these changes will be continuous, however, due to finite observable resolution of space/time, on some scale, changes may be interpreted as discrete.

Regardless of interpretation, since adjacent states of reality are correlated, it is useful to introduce an additional dimension (time) to describe its evolution.

Speed of transformation of energy may then be its speed in time, while speed of transfer its speed in space.

Note that speed of transformation is proportional to speed of transfer of energy at some scale. Therefore, time has a physical interpretation at some scale - ageing of phenomena is strongly correlated with transfer of quanta of energy at some scale, and these quanta may be interpreted as carriers of change or carriers of time.

Note also that transformation can occur at different scales, hence, multiple time dimensions exist with different speeds of information (energy) transfer (generally inversely proportional to scale).

It seems that 4-dimensional manifolds should be used for any scale of energy, however, scale invariant framework can be constructed in which either units will depend on scale or dimensional constants will depend on scale. 
- relatively the same energy relatively oscillates between relative existence and relative non-existence,

- oscillation between vertical energy levels generally involves transformation of scale of angular momentum components (m,v,r), conserving momentum,

- scales of invariance of physical laws are discrete, with exponential progression and may be separated by multiple orders of magnitude.

Relativity of everything indeed has the power to explain everything. Consider relativity in causality. In General Relativity (GR), it is always the clumping of matter (energy) that bends space, however, if causality is relative - sometimes it is bent space that should cause clumping of matter. Of course, one can insist that space is abstract and attribute the energy to exotic matter (ie. dark matter) [bending abstract space] to conserve causality, but is that the proper or most elegant solution, or simply the most elegant patchwork?

And, most importantly, does it lead to better understanding of universes, considering the fact that causality is found to be violated at other scales (universes)?

To me, proper solution is general correlation of past and future states which may be locally absolutized to causality.

Note that distance between past and future is relative. And distance between phenomena is never absolute 0. Every interaction is a relative interaction and is the result of synchronization - correlation in space and/or time. Causality is simply biased (polarized) interpretation of synchronicity.

All interactions or forces in nature depend on distance and all involve correlation. This suggests that distance should be generalized to distance in correlation.

To conserve relativity (existence), forces cannot be exclusively attractive or repulsive and strength of force will sometimes be proportional, sometimes inversely proportional to distance.

Note that exotic matter hypothesis (at least by current interpretations) simply doesn't match observations - ie. cold dark matter halos within galaxies should slow down the rotation of galactic bars but the expected slowdown is not there[1].

There are other problems too[2]. 
These can all be solved by decoupling of space and matter, allowing different densities of space within the galaxy. Equivalent exotic matter here might generally be hot but transforming to cold with coupling, not absolutely free-streaming, rather orbiting around the galactic centre as static particles [forming galactic space].

\subsection{Everything is exchangeable}

Absolute relativity in space introduces problems such as one in relativity of containment. Consider two perfect spheres different in scale and located (centred) at [relatively] the same point in space - how is it possible that a reference frame exists in which the bigger sphere is contained within a smaller one (nondimensional rationality is relative)?

Conservation of relativity thus clashes with the conservation of rationality (intuition).

Seemingly, either non-dimensional rationality is relative or one must be sacrificed for the other.

Here, finite speed of information transfer enables an elegant resolution conservation of both by exchanging relativity in space for rationality in time (subspace).

If two spheres oscillate in time between two scales and one does not discriminate between space and time, both quantities are conserved (rationality in space, relativity in time).

Thus, on absolute scale everything is conserved, but relatively even relativity can be sacrificed.

Multiple interpretations and multiple solutions are common in nature. This problem is no exception. Suppose that everything exists [relatively] simultaneously on different scales - a bigger sphere can then be relatively contained within a smaller one if the smaller one has its identical copy on larger scale.

Note that, if everything must be relative, location and scale of existence must be too, therefore, everything should exist on different scales. This explains self-similarity of universes and suggests that planetary systems such as the Solar System are likely not only similar to atoms (certainly not by coincidence) - they might correspond to specific atoms in relatively equivalent (properly scaled) conditions (pressure/temperature). From some reference frames this might be interpreted as a change in discrete vertical energy level - they have been inflated from standard atoms.

Note that, since existence is relative, existence of containment must be rel- 
ative, so there also must exist a reference frame in which one cannot tell which sphere is contained within the other.

When integrated over time, such state may be interpreted as superposition of different states (spheres), while it's derivative in space is an entirely new sphere (as a result of fusion or superposition of multiple states).

Unless one accepts the notion of multi-scale existence, it might seem that relativity of containment is generally not conserved, even in time. Consider the example of a chicken in an egg - there is no apparent oscillation, chicken might be growing inside the egg and will eventually get bigger than the egg but the egg is not getting smaller and is at no point inside the chicken.

However, another kind of relativity can solve the problem - the egg was once inside the adult chicken. This means there must exist a reference frame relative to which there is no distinction between the two chickens (relativity of identity). Such reference frame does exist and is enabled by the finite resolution (scale) of information carrier particles.

Effectively, both the chicken and the egg are oscillating over time. With absolute containment nothing would be able (required) to grow - neither chicken nor any other universe, with relative containment everything must grow.

Complete relativity and its conservation in reality is what makes everything possible.

Apparently, both GR and QM allow [or are at least partially based on] the existence of an absolute rest frame with constant speed c, completely disallowing any faster means of information transfer on any scale.

Assumptions like that make GR absolutely non-relative and in QM produce many non-intuitive phenomena in space which would otherwise be completely understandable. Even space in GR is non-intuitive in common interpretation it has plastic geometry but no physical properties.

If one does not discriminate between space and time, one could notice that space and time are often inverted in QM.

In example, quantum entanglement between distant particles can be local in time even though it appears non-local in space.

Note that conservation of quantum entanglement requires relative isolation, or non-transformation, of energy. If the state of a particle doesn't change, its speed in time is 0 . Therefore, distance between two entangled particles in time remains the same (relative 0) even if they are separated in space. Such particles obviously must share a time dimension, and in $\mathrm{CR}$, that is a physical dimension on some scale. Strength of entanglement 
here is then proportional to correlation in time.

However, there are no physical point particles in reality, therefore, distance in physical reality is not 1-dimensional, it's volumetric. Thus, correlating volumetric distance with entanglement, with increasing distance in space, the cross-section of time dimension between two particles is shrinking in the middle but the volume (volumetric distance) remains constant.

Wave/particle duality with a change of scale (collapse/inflation of entangled quanta) also becomes intuitive, and a reasonable explanation of quantum tunnelling.

It is reasonable to assume that (default) scale of time relative to scale of atoms is the scale of [rest mass of] photons and gravitons, however, it is not impossible for entangled scales of space and time to be of the same order of magnitude (ie. entanglement of a positron and electron).

Note that speed in space is limited by the specific scale entanglement (it is now obvious that annihilation of matter and anti-matter must involve a change of scale) - due to relativity in density and pressure of space, speed limits will depend on [the scale of] particle energy (the experience of space, or sensitivity to particular energy quanta, is different for particles of different scale).

Energy of larger scale is, of course, generally composed of energy of smaller scale. Therefore, speed limit for such bodies will generally be effectively smaller than for individual constituent particles. Superluminal particles must exist at some smaller scale, however, as noted before, observers are always limited in resolution and may not be able to directly detect them.

However, collapse of quantum entanglement (state superposition) in CR is breaking [on one end] and contraction [to other end] of distance (time dimension) between particles and speed of that contraction becomes superluminal at some distance in space. This is then indirect observation of superluminal transfer of information unless contraction of time is interpreted as contraction of space.

If space is real, there is no reason for intrinsic coupling of matter and space curvature, therefore a reference frame must exist where there is no such coupling - dark matter can then be interpreted as direct evidence for real space.

It is intuitive for space to have relative density and pressure which can, as vacuum energy, attract particles that are sensitive to such density and pressure. And these particles may have their own space coupled with them. Reality thus becomes intuitive even on small scales - electron can rotate faster than c (although this can be interpreted as rotation of space, with constituent particles 
of electron matter or charge being at rest relative to that space), have a real radius and real orbits inside the atom.

With atomic nucleus allowed to have its own rotating space the electrons do not emit energy when in a specific orbit, even in corpuscular form - they are at rest relative to that space.

Relative electric permittivity (dielectric constant) and magnetic permeability of materials now make physical sense and can be correlated with properties of space of atomic nuclei.

Current (absolute) vacuum electric permittivity and magnetic permeability would be no different - these are also properties of space.

One might argue that the existence of physical space has been disproved with experiments, however, that is not the case - various interpretations of aether have been disproved[3]. While space in Complete Relativity has some similarities with, what was originally called, aether, it is not the same phenomenon. One could consider the space in CR to have the same geometry as space in GR, however, with real density and pressure instead of being nothing more than non-intuitive abstract geometry. There are differences though - GR requires modification to become completely relative (implement scale invariant curvature and solve gravitational anomalies).

\subsection{Everything has an angular momentum (everything has mass)}

All mass can be correlated with intrinsic and inheritable spin and orbital angular momenta of space at some scale.

For something to exist and be observable, it must have a momentum at some scale.

Each angular momentum is composed of spin momentum quanta at some scale.

Capacitance of space allows transfer of momentum, but also its conservation. Any mass (energy) at relative rest is a conserved momentum in form of local spin momenta. The rest energy is thus locally conserved kinetic energy and a reference frame must exist relative to which the energy is in motion.

Individual momenta may entangle to form larger structures, and with enough energy, fuse to form larger momenta. Since momenta are quantized not every momentum is stable, and even the stable ones are only relatively stable with a difference in decay rates.

Obviously, rest mass is relative and will not be absolutely equal even between individual particles of the same species. 


\subsection{Everything is entangled on some scale (all correlation is physical on some scale)}

All physically possible entities exist and are physically connected (entangled) on some scale (everything exists on various scales). In absolute reality this might imply everything was initially condensed into a singularity, but in relative reality this might be interpreted simply as natural manifestation of correlation, a consequence of required relativity (ie. of abstractness). However, condensation into relative singularities is not impossible, will exist and will affect entanglements.

The strength of entanglement between two entities depends on entangled energy or distance in correlation:

$$
\propto \frac{1}{r^{n}} \propto \frac{\Delta E}{E}
$$

where $\mathrm{E}$ is the energy of entangled entity, $\Delta \mathrm{E}$ is entangled energy, while $\mathrm{r}$ is distance in correlation (which will generally reduce to distance in space or time).

Entanglement is weak if the observer can break it with asymmetric appliance of energy.

Note that entanglement can be invariant to distance in space if entanglement is physically manifested in time and [volumetric] distance in time remains unchanged.

Strong entanglement is established when it becomes physically impossible for an observer to disturb the proportionality of $\Delta \mathrm{E}$ and $\mathrm{E}$. This occurs when applied energy is distributed on both, particles and the time (space) dimension connecting (entangling) them. At some point, however, applied energy may produce (inflate) new pairs of particles.

Note that strong entanglement is relative to an observer (scale of energy) and can be broken by a less limited one.

\subsection{Everything is intrinsically polarized}

Since energy requires spin which can have different orientations, it is intrinsically polarized (although the amount of polarization is relative). Any apparently neutral system is thus a configuration of balanced polarized systems and must stem from homogeneous polarity distribution, polarity oscillation or confinement.

High frequency oscillations may be interpreted as superposition of adjacent states in some reference frames, but in reality the period of oscillation is never absolute 0 .

\subsection{Physical laws are scale invariant}

In nature, distance is quantized, but the size of quanta is not constant. Energy generally oscillates between stable discrete energy levels. These energy levels 
can be horizontal (as described by QM) or vertical. Horizontal energy levels differ in the amount of energy but generally not in the order of magnitude, vertical levels generally differ by multiple orders of magnitude.

Although universes at different scales may be in different equilibrium states, applying energy, state on one scale can be made locally relatively equivalent to a state on another scale.

Here, state generally refers to scale relative pressure (or temperature) and density, which generally differ between vertical energy levels.

Temperature and density on standard scale are proportional to kinetic energy and density of atoms, respectively, on a higher vertical energy level this may be kinetic energy and density of planetary systems, on a lower energy level energy and density of photons.

Physical laws of nature are thus [relatively] scale invariant, with stable scales appearing at discrete points between intervals of generally exponential progression.

Elementary particles are strongly relative to reference scale. From a smaller scale they will evidently be composite and differ from each other, while from a larger scale they may even be unobservable (non-existent) individually.

\subsection{Hidden variables always exist}

With no absolutely elementary phenomena and with inherent (but not absolutely constant!) limits in observation, hidden variables must exist. Therefore, non-intuitive reality suggested by QM must be taken relatively. As stated already, treatment of all constants as relative constants can make QM much more intuitive, but it does not end there.

It should be noted that intuition too must be relative. If common intuition (hidden variables) cannot be observed, it is valid to conceptualize non-intuitive reality (and declare this non-intuition as natural or intuitive from some reference frame), however, one cannot claim such reality is absolute - there are always possibilities for different interpretations.

One other concept problematic for common intuition is the concept of spinors, or generally, algebra not correlated with geometry, or, not correlated with intuitive geometry. Sure, one can use two-dimensional planes (complex numbers) to represent phenomena [occurring in three-dimensional or four-dimensional space], and calculations within such frameworks may give consistent and good results matching the outcome of experiments, but that does not mean reality is absolutely two-dimensional or non-intuitive on a particular scale. Geometric and 
[commonly] intuitive interpretation in three dimensions is always possible[4] even if it may require hidden variable[s]. And if complete relativity implies such variables must exist, and, if one strives for deeper understanding of reality, it would be, not only counter-intuitive, but counter-productive to discard them simply because mathematics without them works and/or is more elegant. Especially considering the real possibility, implied by $\mathrm{CR}$, that one might be able to relatively observe the hidden variable on another scale of a particular state. Furthermore, mathematics working today does not mean it will work tomorrow - any hidden variable is variable after all.

\section{On stability of equations}

Equations are very useful and powerful constructs of a mathematical language. They can lead to new insights on details and plausibility of hypotheses, but can also be very deceiving.

All dimensional constants are relative. Therefore, more constants the value of equation depends on, more unstable it is.

Dimensionless constants are generally more stable, but even these are not absolute.

With everything being relative, a presumption of absoluteness will eventually lead to discrepancies in measurement and misinterpretation of reality.

\section{Definitions}

Here are the definitions of terms and expressions that may be used here and other papers and articles in CR context. Note that these may be different than standard or common definitions in use.

The definitions form a crucial part of the theory and should not be skipped or considered less important than any other chapter if one aims to understand the theory.

\section{1 $\mathbf{n}_{t h}$ order observer}

In the context of quantization (measurement) of physical phenomena, observer is an entity performing the measurement.

The order of the observer is a relative sum of the number of interactions in the act of measurement which affect its result.

In example, 1st order observer may be the information carrier (radiation) particle, 2nd order observer is then the radiation detector, etc.

Every observation is measurement, albeit not always a conscious one. Each measurement affects all interacting entities. 


\section{$4.2 \mathrm{n}_{t h}$ order interaction (action and reaction, energy os- cillation)}

Consider the forces in Newton's law of gravitation:

$$
\begin{gathered}
F=\frac{d}{d t} p=\frac{d}{d t}(m v)=m a=G \frac{M m}{r^{2}} \\
F_{1}=m_{1} a_{2}=m_{1} \frac{m_{2} G}{r^{2}}=m_{2} a_{1}=m_{2} \frac{m_{1} G}{r^{2}}=F_{2}
\end{gathered}
$$

Here, forces acting on bodies $\mathrm{m}_{1}$ and $\mathrm{m}_{2}$ are equal and have opposite direction, as expected for forces of action and reaction. Note that these are actions and reactions between bodies of standard matter at distance.

In General Relativity there is no action and reaction between the two bodies, but effectively between continuous space (more precisely, geometry) and a particular body (or energy, in general). And it is not an action and reaction at distance (distance is assumed to be equal to absolute 0 ).

Note that in both cases action/reaction is instantaneous, so even in Newton's gravity distance between the sources is effectively 0 for whatever is mediating the force, only the 1st order sources of interaction differ.

In Complete Relativity there are no absolute zero and infinite distances, thus every action is action at a distance which may only relatively be equal (set) to 0 .

If gravitational entanglement is understood as physical entanglement at some scale and it is never absolutely zero between two bodies one can understand why are the forces acting apparently instantaneously - particles mediating the force are constantly streaming between two bodies (forming subspace in space, which may be interpreted as another dimension, ie. time). So even though they are travelling at finite speed (which in GR would have to be the speed of light $\mathrm{c}$ if these particles are massless) the action appears instantaneous. Changes in gravity at sources, however, obviously propagate at the speed of carrier particles.

These particles are not absolutely massless, so they too are sources of force and are mutually entangled. At appropriate density they form a medium enabling wavelike transfer of information (energy). The wave can be considered massless relative to the medium (energy is stored in temporarily excited quanta of the medium), however, quanta of excitement are not massless and are also mutually entangled.

Since wavelike behaviour of energy is synchronized excitation of quanta of smaller scale energy, a wave of energy can be considered as superposition in time (distance between entangled quanta in time is relative 0). Then, if momentum is disturbed, this superposition in time can, partially or fully (in case of strong entanglement), collapse to superposition in spacetime (distance between entangled quanta in space also becomes 
equal to relative 0). Note that this can be interpreted as collapse of fermionic pairs into a bosonic state.

In GR however, space is not quantized - no constituent quanta (gravity carrying particles) of spacetime are defined (geometry is not interpreted as physical).

The two bodies have an effect on space (and vice versa) but they also affect, albeit indirectly in GR, each other.

In CR, with applied scale invariance, it is obvious that even the interaction between quanta of space and quanta of bodies must also be an action at a distance, albeit this distance is orders of magnitude shorter than distance between the bodies (without applied scale invariance on distance) and may be considered infinitesimal.

The sources of force of action and reaction are thus relative to scale - measuring on larger scale it may be more appropriate to attribute the sources to bodies, while on lower scale the quanta of space may be interpreted as such.

From a 3rd perspective one may consider the action between a force carrier particle in space (even if it is a bound static particle with potential energy) and a body as 1st order interaction, and the interaction between two bodies as the 2nd order interaction.

One may also consider the 1st order interaction as relatively instantaneous, 2nd order occurring at some speed c, 3rd order at even some lower speed, etc.

In any case, distance is quantized and there is no absolutely instantaneous and equal reaction to action (it requires quantum of distance equal to 0 , or, equivalently, infinite speed of carrier particles).

Causality is a relative illusion, created by high synchronicity (correlation) of events.

The relativity of sources (force carriers) and distances has an important consequence on the law of action and reaction - it needs generalization:

$$
\int_{t=0}^{T}\left[\vec{F}_{1}(t)+\vec{F}_{2}(t)\right] d t=0
$$

Instantaneous action and reaction is thus a special case of action and reaction impulses, where the period of energy oscillation $\mathrm{T}$ is compressed to an instant a single elementary quantum of time $(\mathrm{dt}=\mathrm{T}=1)$ :

$$
\overrightarrow{F_{1}}+\vec{F}_{2}=0
$$


Note the equivalence of distance in time and space of different scales in the 1st order interaction (GR) distance in space is 0 , while in the 2 nd order interaction (Newton) distance in time is 0 . Increase of distance in space between two bodies at 2 nd order scale proportionally increases the distance in time at 1 st order scale.

Also note that, although not required, it is not forbidden for action and reaction to be simultaneous, nor it is forbidden for reaction to precede action, allowing relativity of cause and effect (something that is, with absolutely constant c, forbidden in GR, but required in $\mathrm{CR}$ ).

In CR thus, causality is not absolutely fundamental nor intrinsic - it could be understood as a result of force, relatively emerging (or evolving) between correlated (entangled) phenomena decreasing distance in space and/or time. However, proper interpretation is that causality is simply localization of synchronization (synchronicity) of events in a polarized reference frame.

Violation of [absolute] causality will exist on all scales of energy, but amount will differ between the scales. However, the amount has to oscillate too and will correlate with changes in polarization of space (ie. electro-magnetic).

The equation (1.1) is equivalent to momentum pulse (energy) reflection:

$$
\int_{t=0}^{T}\left[\overrightarrow{F_{1}}(t)+\overrightarrow{F_{2}}(t)\right] d t=\int_{t=0}^{T}\left[\frac{d \overrightarrow{p_{1}}}{d t}+\frac{d \overrightarrow{p_{2}}}{d t}\right] d t=\int_{t=0}^{T}\left[d \overrightarrow{p_{1}}+d \overrightarrow{p_{2}}\right]=\overrightarrow{p_{1}}+\overrightarrow{p_{2}}=0
$$

With $\mathrm{T}>\mathrm{dt}>0$, action and reaction becomes a manifestation of energy oscillation.

However, entanglement can be relatively broken (reduced to relatively infinitesimal strength) and reaction might be delayed, may not affect the source of action or might not even occur (if the action is locally interpreted as reaction).

All bodies having rest mass possess capacitance and reaction to the source will eventually come, even if from another body, if that capacity is not in equilibrium state.

The body producing the reaction will generally be relatively the same [scale] as the body acted upon, thus, allowing relativity, this can even reduce to instantaneous action and reaction in some reference frames.

However, the reaction may be fragmented and carried by diverse force carriers following multiple different paths.

Such nature of non-apparent oscillation stems from different scales of energy quanta enabling diversity and evolution of complex forms of energy, its conduction and transformation. 
The original action may be the reaction to action from a 3rd body in which case the source returns to equilibrium after the action and the body may be interpreted as simply the energy conductor (momentum carrier).

In reference frames where force of reaction is absent, the interaction may be more appropriately described through conservation of momentum:

$$
p_{1}+p_{2}=C
$$

Note also that, even if all energy must oscillate, this is never absolutely identical energy - never will absolutely the same body experience the reaction (zero in equations above is also a relative zero).

\subsection{Superposition}

Superposition is a special state of a system which is a combination (generally sum) of multiple possible states.

With each state having a unique physical interpretation, in reality, only effective superposition is possible - relative to spatial or temporal resolution (scale) of the [1st order] observer.

If the observer cannot resolve individual states in superposition it will strongly affect it by observation (measurement). The superposition of states will then generally collapse to either:

- an average value of oscillation between multiple states in time,

- a discrete state, in case of oscillation between discrete states,

- an entirely different physical state, as a result of spatial transfer/transformation of superposition forming entities.

Note that states in superposition are correlated (entangled, physically at some scale).

\subsection{Universe (U)}

Commonly, Universe is defined as simply everything that exists, has existed and will exist. Thus, that definition refers to an absolute (unobservable) universe (Universe). In context of $\mathrm{CR}$, much more useful is a relative, finite and observable universe, and that could refer to any distinct form of energy.

However, in context of CR, a universe, by default, refers to a particular scale of energy. Generally, these scales will be analogous to discrete vertical energy levels and will correspond to energy magnitudes of elementary (stable) particles on that level (the building blocks of intermediate unstable or evolving scales of energy).

These universes are correlated, but generally not evolutionary synchronized. 


\subsubsection{Charge (c) and scale (n.m) of a universe}

Universe will typically refer to a scale. If it refers to a specific particle or a system of particles, charge may also be specified. There are two equivalent notations:

$$
\begin{array}{r}
c U_{n . m}=c U(n . m) \\
\mathrm{n}=\text { vertical scale of the universe }(0=\text { reference universe) } \\
\mathrm{m}=\text { scale of the (horizontal) sub-universe } \\
\mathrm{c} \in\{-,+\} \\
\mathrm{n} \in \mathbb{Z} \\
\mathrm{m} \in \mathbb{Z}
\end{array}
$$

The $\mathrm{n}$ scales are usually chosen in such a way that $\mathrm{U}_{-1}$ scaled energy quanta form the space (medium) of $U_{1}$ bodies, while $U_{-2}$ forms the space (medium) of $\mathrm{U}_{0}$, etc.

If $\mathrm{m}$ is specified, the $\mathrm{U}_{n . m}$ denotes the sub-universe of scale $\mathrm{m}$, larger than $\mathrm{n}$ but smaller than $\mathrm{n}+1$.

If specified, charge c denotes the polarization, generally, electric.

Usually, $\mathrm{U}_{0}$ scale refers to scale of protons and electrons, in which case $\mathrm{U}_{1}$ would refer to magnitudes of stars and planets.

\subsection{Standard particle}

Standard particle (ie. standard electron) refers to a particle as defined by the standard model in physics.

\subsection{Elementary particle}

An elementary particle on small scales is a relatively stable particle with generally unresolvable constituent particles. On large scales, relative equivalents of these may be considered elementary, even though their structure may be resolvable by the observer.

Appropriate elementary particles of scale $\mathrm{U}_{0}$, in most cases, should probably be protons and electrons.

\section{7 $\quad$ Existence}

Distinct forms of existence are distinct forms of energy. Existence requires energy. All discrete quanta of energy are produced (inflated or deflated) with changes in momentum.

For any form of energy to exist, there must exist a reference frame relative to which its angular momentum $(\mathrm{L})$ is greater than 0 :

\subsubsection{Single entity}

$$
|L=m v r|>0=>|m|,|v|,|r|>0
$$

where $\mathrm{m}, \mathrm{v}$ and $\mathrm{r}$ are momentum mass, velocity and radius, respectively. 


\subsubsection{System of $n$ entities}

$$
L=\sum_{i=1}^{n} L_{i}
$$

\subsection{General oscillation}

With no absolute constants, everything must oscillate, even oscillation itself.

Change of a variable in dimension $\mathrm{x}_{i}$ may thus generally be described with the appliance of the following operator:

$$
\begin{array}{r}
\frac{d}{d x_{i}}=a_{1} f\left(\omega_{1}\left(x_{i}+\phi_{1}\right)\right)\left[1+a_{2} f\left(\omega_{2}\left(x_{i}+\phi_{2}\right)\right)\left[1+a_{3} f\left(\omega_{3}\left(x_{i}+\phi_{3}\right)\right)[1+\ldots]\right]\right] \\
\mathrm{f}=\text { oscillating function } \\
\mathrm{a}_{j}=\text { amplitude of } \mathrm{j}^{\text {th }} \text { order oscillation } \\
\omega_{j}=\text { frequency of } \mathrm{j}^{\text {th }} \text { order oscillation } \\
\phi_{j}=\text { phase shift of } \mathrm{j}^{\text {th }} \text { order oscillation }
\end{array}
$$

where, generally, $\mathrm{a}_{j}<1$ for $\mathrm{j}>1$.

Multiple dimensions are generally entangled, so $f$ may be $f\left(x_{i}, \ldots, x_{n}\right)$.

\subsection{Frequency of existence}

Existence is relative and it depends on the scale of a reference frame (one cannot have the ability to measure energy at any scale possible), but may also oscillate between energy levels.

For a particular order of general oscillation and its period $\mathrm{T}_{x}$, frequency of existence is:

$$
f_{x}=\frac{1}{T_{x}}=\frac{1}{\Delta T_{1}+\Delta T_{0}}
$$

where $\Delta \mathrm{T}_{1}$ is the average lifetime on a larger scale and $\Delta \mathrm{T}_{0}$ is the average lifetime on a smaller scale. Generally, $\Delta \mathrm{T}_{0}$ may be $« \Delta \mathrm{T}_{1}$, and $\mathrm{T}_{x}$ may be approximated with $\Delta \mathrm{T}_{1}$.

Frequency of existence is a property of species but it generally fluctuates in value between individual forms of energy.

Note that oscillation must be relative and will not be apparent from all reference frames. Sometimes, energy will be observed as a pulse or a relatively non-changing (weakly evolving) phenomenon.

Oscillation can also be disturbed and fragmented.

In example, energy in the form of a living being might be observed as a pulse of energy growing from conception to a maximum then decaying until death. Note, that no form of energy grows from nothing or decays to nothing. Growth is synchronized with defragmentation or accumulation while decay is synchronized with fragmentation or dissipation of energy. 


\subsection{Relativistic uncertainty}

As all observation is physical on some scale, no measurement can be performed without affecting the subject of measurement.

Measurements will also necessarily be limited in resolution and for all observers there will exist a threshold of possible precision (inherent uncertainty).

Measuring complementary variables such as energy and time, or components of angular momentum, on smaller scales, precision in one will have to be sacrificed for precision of the other component.

This is commonly formulated as the Heisenberg uncertainty principle:

$$
\sigma_{x} \sigma_{y} \geq \frac{1}{2} \hbar
$$

However, if $\hbar$ is interpreted as an absolute constant, or, if dimensional units of components are not scalable, the interpretation is limited to a particular scale of space.

Per CR postulates, there can be no absolute point particles, thus, volumetric position in reality is not limited to waves and large scale objects.

Relativistic uncertainty is:

$$
\begin{aligned}
\sigma_{x} \sigma_{y} & \geq \frac{1}{2} \hbar_{n} \\
\hbar_{n} & >0
\end{aligned}
$$

where $\hbar_{n}$ is relative to scale $n$.

$$
\lim _{n \rightarrow-\infty} \sigma_{x} \sigma_{y}=0
$$

In conventional quantum theory (QM) [virtual] particles are popping in and out of existence all the time, which is allowed by time-energy uncertainty. In CR, existence is relative and this is simply oscillation of energy between different scales. This is generally a consequence of exchange between angular momentum components.

In example:

$$
\Delta E \Delta t \geq h
$$

should then be interpreted as:

$$
\Delta\left(m v^{2}\right) \Delta t=\Delta\left(m v^{2}\right) \Delta\left(\frac{2 \pi r}{v}\right)=\Delta(m v) \Delta s=\Delta p \Delta r \geq \hbar
$$

Exchange of angular velocity $\mathrm{v}$ for rest mass $\mathrm{m}$ may result in a jump to a higher vertical energy level - appearance of energy on a larger scale. If the rest mass 
on that particular scale was previously considered a relative 0 , this may be interpreted as relative violation of energy conservation, allowed as long as the increase in energy (inflation of $\mathrm{m}$ ) is inversely proportional to particle stability $(\Delta \mathrm{t})$.

Period of stability is thus the period of angular momentum, and in reality, energy is conserved.

\subsection{Zero}

Absolute zero value of any variable represents non-existence of phenomena in physical reality. However, in some cases, zero should be interpreted as a relative zero.

In example, if a form of energy behaves as a wave on one scale, its energy may be equalized with frequency, in which case it may be assigned zero mass, however that zero should be understood as relative to excited medium - at some scale there are particles with momentum increased proportionally to that frequency.

\subsection{Infinity}

Absolute infinity has no physical interpretation. While absolute infinities are useful in mathematics, in some cases, these should be interpreted as relative infinities.

Consider the relativistic Lorentz factor applied to mass of a particle:

$$
m=\frac{m_{0}}{\sqrt{1-\frac{v^{2}}{c^{2}}}}
$$

By the equation, at speed c, mass of the particle would have to be absolutely infinite, implying that absolutely infinite energy is required to accelerate the particle to $\mathrm{c}$.

However, in CR, the speed limit c is relative to [structure of] space and, even in GR the speed limit is equal to c only in case of flat space geometry. Adding energy to the particle will, at some large but finite value, start to significantly affect the structure of space and particle could, with asymmetry in density, even exceed the speed c.

Speed c is also considered to be the speed of massless particles in QM. However, standard massless particles will also slow significantly below c in strong gravitational potential (although local observer would measure no slowdown as units of distance are decreased proportionally).

Also, constituent quanta of space are of particular scale and not all scales of energy are equally sensitive to these (pressure/density of space is relative), 
thus, speed c can be exceeded by energies of extremely small scale too (although such energies may be unobservable).

Although momenta in a universe will be inevitably limited, in CR, c cannot be an absolute constant. Even if $\mathrm{c}$ is interpreted as proportional to a ratio of units of distance in space and time and these units scale with geometry (as in GR) conserving that ratio, the geometry itself must be relative.

\subsection{Graviton (quantum of spin momentum, [closed] grav- itational field tube, quantum of energy, soul)}

Graviton is a real or effective source of a general force field - a more or less polarized quantum of space at some scale.

Real gravitons are sources of general force, while effective gravitons are induced by real gravitons and are carriers of the force through the general field.

Generally, real graviton will be considered as an elementary particle, while effective graviton is composed of real gravitons of smaller scale.

The force (vacuum, or curvature in some interpretations) is greatest in a real graviton and decreases exponentially with distance of effective gravitons from the real one.

Generally, graviton may be interpreted as a low matter density (high vacuum), rotating region of space at some scale. The associated well of gravitational potential may be considered its private space, however, that privacy falls off with distance.

In CR, everything must have an angular momentum from some reference frames. This is a consequence of intrinsic rotation of space on all scales.

Its shape depends on electric charge and spin momentum (which may be quantized from some reference frames). Relativistic momenta may further distort the shape of a graviton.

Graviton is generally a composite of at least 3 components - 1 neutral space (vacuum) and 2 charged momenta, forming a torus with size of openings proportional to amount of electric polarization.

Charge carrier particles are generally asymmetric in energy and may have significantly different momenta in polarized states. 
Physics of a theory of everything cannot be reserved for particles and their interactions at specific scale. From certain reference frames, even living beings are particles, and vice versa.

Distinction between living and non-living forms of energy is very relative and physics will necessarily merge with biology in a successful attempt to understand the universes.

In follow-up papers I hypothesize that real graviton is, considering its nature, also a quantum of consciousness and I find it appropriate to use the term "soul" as its synonym.

\subsubsection{Physical interpretation}

Assuming the scale of a graviton is $\mathrm{U}_{n}$, constituent quanta of its space will be of scale $\mathrm{U}_{n-2}$. If these are inflated to scale $\mathrm{U}_{n-1}$, such quanta will be considered as [mass] coupled with quanta attracted by the graviton (constituent quanta).

Graviton can exist on different vertical energy levels (scales), however, on some scales the neutral (gravitational) component might dominate, on others its nature may be dominantly electro-magnetic.

While real gravitons induce gradients in vacuum density (or space curvature), space inside of a real graviton may be globally flat with extremely low density and temperature of [whatever is interpreted as] matter. However, high curvature should be present at the membrane, which then represents a source of extroverted gravity of a graviton.

While temperature and density are low globally, high temperatures and densities are possible and do exist on smaller scales. The particles are often in condensed (bosonic) states and grouped in quantum vortices (galaxies).

The shape of a graviton is generally a torus, with an intrinsic spin momentum. This spin momentum is relatively quantized with constituent smaller scale spin momenta, forming smaller scale sources of force (these are generally centres of quantum vortices, where most energy is concentrated inside graviton space). Thus, large scale spin momentum (of the graviton) is strongly correlated with small scale momenta.

Shape of a graviton is shown in Fig. 1, where the surface of a torus represents its effective boundary.

As a discrete quantum of vacuum, graviton must have an effective membrane.

Note that it is relatively easy to maintain existing conditions inside the graviton, as accumulation of particles is extremely hard due to flat space and low-density of matter.

Any particle having a momentum perpendicular to the graviton may be accelerated inside (in most cases, the trajectory of the particle may be 


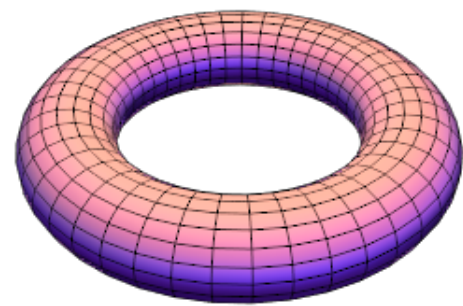

Figure 1: General shape of a graviton

simply bent around the graviton surface), but will be equally decelerated again, and will exit the graviton with the same momentum. Collisions will be hard even if existing and passing particles are of the same scale, but if existing particles are of smaller scale (discrete vertical energy levels differ in energy by multiple orders of magnitude), accumulation becomes almost impossible. In that case, graviton is relatively transparent (transparency is dependent on energy scale).

However, nature of a graviton can change with changes in its spin momentum.

Assuming that, in neutral state, escape velocity of the graviton is larger than the speed limit, a graviton can become electrically polarized if its rotation (rotation of its constituent space, or orbital velocity of quanta forming that space) decreases enough that escape velocity becomes smaller or equal to the speed limit.

Its rotation is thus proportional to its gravity and this becomes exchange of gravitational potential for electro-magnetic potential. Decreased rotation of a graviton is decreased orbital angular velocity of its constituent $\left(\mathrm{U}_{n-2}\right)$ quanta. This is coupled with decrease of constituent $\left(\mathrm{U}_{n-1}\right)$ spin momenta, and these will, to conserve total momentum, increase orbital angular momentum. The increasing $\mathrm{U}_{n-1}$ temperature and asymmetry in spin momenta will eventually lead to separation of entangled spin momenta (creation of ions) and the graviton itself becomes a charged particle and a source of a magnetic field.

If neutral (gravitational) energy is decreasing the polarized energy must be increasing and that is the energy in magnetic field lines (tubes, or toruses). Magnetic field lines are thus induced polarized effective gravitons perpendicular to the original graviton and space in these lines is formed by particles of $\mathrm{U}_{n-3}$ scale.

The charged graviton will now effectively attract oppositely charged gravitons due to decreasing distance in correlation (increasing energy in connecting 
magnetic field tubes) with interaction, while like charges will be effectively repelled by opposite action.

On neutral particles, net effect of polarized space will be a relative (oscillating) zero unless the force is strong enough to break the entanglement of constituent charges.

Note that polarization is concentrating constituent particles to a 2dimensional ring, while neutralization is expanding it to more spherical shape. To conserve volume, expansion will be decreasing the thickness of a torus, converging to a 2-dimensional sphere.

Note also that conservation of that volume can also be interpreted as conservation of correlation between opposite sides of the membrane. If the symmetry of the membrane would increase, the volume would decrease. Note that recent analyses[5] have shown that the shape of the local universe is consistent with that of a torus[6]. Considering its characteristics, it must be a [large scale] graviton.

If distances between galaxies (large scale quantum vortices) are increasing, this graviton is increasing its internal flatness and must be changing shape.

Increasing rotation of space thus increases mass (gravity) of constituent matter, reducing its angular speed and charge, while decreasing rotation of space does the opposite.

Note however that, as stated before, cause and effect are relative and synchronization is a better term.

Decreasing rotation of space is not only synchronized with inflation of constituent quanta but generally also with their coupling with $\mathrm{U}_{n-1}$ matter.

\subsubsection{Interaction of gravitons}

When bosonic, the constituent (uncoupled) quanta of one graviton will weakly interact with constituent quanta of another graviton. However, in case of stronger entanglement the two may form superposition in space and probability for interaction might increase.

Superposition is, of course, relative, and if gravitons are of different scale, orbital radii of constituent quanta will be different.

Note that, in that case, the outer real graviton may be effectively shield- 
ing the gravity of the inner real graviton. This is a consequence of strong entanglement, where induced effective gravitons by the inner graviton have decreased range and are confined to space between the two real gravitons. Confinement is relative and some inner gravity will leak, with highest probability at the poles.

\subsubsection{Acquisition of matter (coupling with matter, acquisition of smaller scale energy quanta)}

A naked (uncoupled) $\mathrm{U}_{n}$ graviton will effectively attract particles of $\mathrm{U}_{n-1}$ scale. There are now two possibilities on the effect of total gravity:

1. with inflation and coupling of a constituent $\mathrm{U}_{n-2}$ graviton with $\mathrm{U}_{n-1}$ particle, one graviton is shielding the other, energy relatively equal to energy of $\mathrm{U}_{n-1}$ particle is confined and there is no increase in gravity of the well with acquisition of $\mathrm{U}_{n-1}$ matter,

2. there is no shielding and total gravity of the well is increased with acquired matter.

Note that 1 . possibility may be interpreted as one particle giving mass to the other. Regardless of the outcome, the interaction should affect the range of gravity of the system. Constituent gravitons of $\mathrm{U}_{n-1}$ scale are of $\mathrm{U}_{n-3}$ scale, thus, one range is being replaced with the other.

Note that each graviton has finite capacity for coupling - number of constituent quanta is not infinite.

Note also that violation of energy conservation in case of shielding is relative - even though gravity may be unchanged (relatively) the total energy is conserved by compression (confinement) and will be released with decoupling.

Since real graviton represents a maximum of gravitational potential it will form a discontinuity in the system. Electro-magnetic nature of a graviton will concentrate polarized matter in the centre, while neutral matter can start concentrating there with collisions.

Coupling of standard matter (ie. standard atoms) with the gravitational well of a large scale graviton is the coupling of static graviton neutrinos or static [dark] photons (constituent quanta of space of the large scale graviton) with this matter.

However, for a large body (composite of atoms) in the gravitational well it is convenient to consider it coupled with the effective large scale graviton, rather than with individual small scale static particles. 
In that case, mass and velocity of the effective graviton are total mass and average velocity of constituent static gravitons, respectively, while the effective graviton forms the toroidal [large scale] quantum of space which the coupled standard matter is traversing in its orbit around the real graviton.

If the whole observable universe is a part of a large scale graviton, galaxies and planetary systems may be the result of inflation and coupling of its constituent quanta. One of my hypotheses is that a discontinuity between inner and outer core of a planet represents the radius of a real graviton that has been inflated from the scale of a standard atom, or even a much smaller scale.

For stars, I hypothesize multiple such gravitons in superposition. This is all further investigated in follow-up papers.

\subsection{Static particle}

Particles forming space of a graviton of scale $\mathrm{U}_{n}$ are particles of scale $\mathrm{U}_{n-2}$. These are entangled with the graviton and any changes in graviton momentum will be reflected in momenta of these constituent particles. The particles are orbiting the graviton and their density is generally decreasing exponentially with distance from the graviton. Orbital speed is equal to speed limit in space for particles of $\mathrm{U}_{n-1}$ scale. However, the particles will get bound to $\mathrm{U}_{n-1}$ scale matter captured by the gravitational well, exchanging orbital velocity for spin ( $\mathrm{U}_{n-1}$ scale) momentum.

Due to their limited range and significant orbital angular momenta the constituent particles will hereby be referred to as static particles (generally, static neutrinos and static photons).

These particles may be interpreted as hot dark matter while uncoupled, however, with coupling, their momenta will be transforming to cold Keplerian momenta.

\subsection{Graviton tube (wormhole, quantum of entanglement)}

Graviton tube is a physical manifestation of entanglement (correlation), space connecting two entangled gravitons. In one interpretation, the volume of that space (volumetric distance) is proportional to distance in correlation (inverse of strength of entanglement), however, the tube is relatively hollow and energy is mostly concentrated on the membrane.

With no additional energy or disturbance of gravitons, volumetric distance will remain the same regardless of spatial separation. The tube can be considered as subspace or an relatively isolated dimension of space. 
The tube may be considered as elementary quantum of continuous space, however, in reality it is a sum of constituent smaller tubes. The tube(s) may also be generally curved (entangled particles may not be connected by the shortest path possible in flat geometry).

Consider magnetic field lines connecting opposite poles - if these are tubes of entanglement, they obviously do not follow shortest paths and may be compressed and expanded.

Of course, these may be shortest paths possible considering conditions on field formation and one can model entanglement of poles with geometry where these paths are shortest.

\subsection{Gluon}

Gluon is a superposition of one or more pairs of gravitons.

\subsection{Gluon tube}

Gluon tube is space connecting two entangled gluons. It is a superposition of graviton tubes.

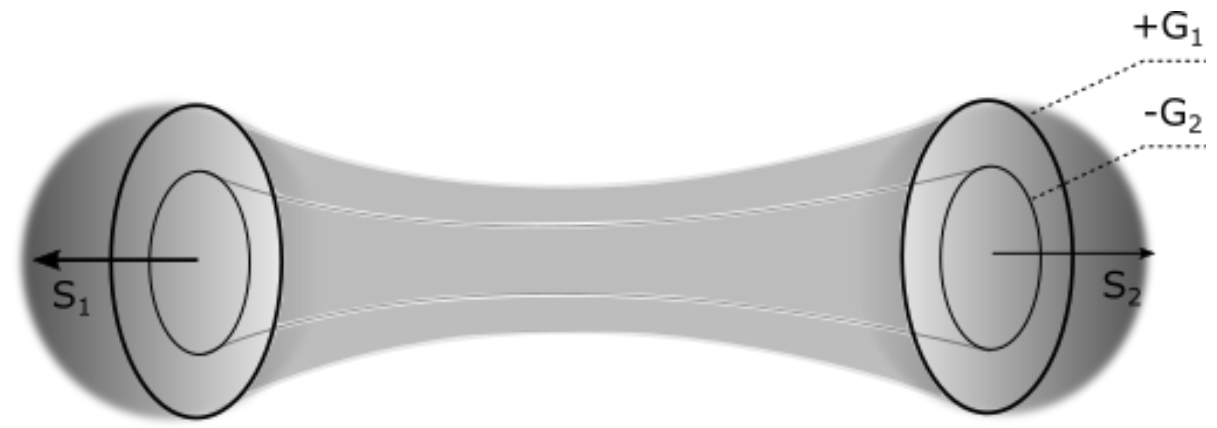

Figure 2: Gluon tube

Fig. 2 shows the gluon tube, with induced cross-sectional capacitance due to spatial separation of gravitons $\mathrm{G}_{1}$ and $\mathrm{G}_{2}$.

\subsection{Gravitational maximum (g-maximum, [relative] event horizon, primary soul)}

Gravitational maximum is a real graviton having maximum potential (energy) of a gravitational well. 
When multiple gravitons are in superposition they will spin around the same central region but their mass radii will generally be spatially separated. Even though each mass radii will represent a local maximum, gravitational maximum is the graviton with maximal mass (energy) of the system.

In equilibrium this will generally be the outermost graviton as lower energy gravitons with larger radii are less stable and are likely to collapse (localize) the wave-like (or cloud-like) spin to orbital angular momentum (corpuscular orbit).

Radius of a graviton will thus generally be proportional to its energy.

Energy of a gravitational maximum is proportional to capacity of the associated gravitational well for coupling with matter.

Note that capacity is scale relative. The capacity of a $\mathrm{U}_{n}$ graviton for coupling with $\mathrm{U}_{n-1}$ mass may be full, but the capacity of $\mathrm{U}_{n-1}$ wells for $\mathrm{U}_{n-2}$ mass may be not.

However, note that constituent quanta of space of a $\mathrm{U}_{n}$ graviton are $\mathrm{U}_{n-2}$ particles, exactly the mass scale the constituent particles of $\mathrm{U}_{n-1}$ wells should couple with. Thus, the capacitances of different scale here are correlated (the coupling correlation is manifested as attractive force) and what is considered as a component of space in one reference frame may be interpreted as acquired matter in another.

The question is what happens to wells at full capacity and can they be over-capacitated? This is related to the problem of dark matter and changes in energy levels, and is discussed later.

\subsubsection{Biological physics}

The acquisition of matter by the naked particle can be interpreted as an act toward symbiosis of smaller scale and larger scale mass. The gravitational well of the maximum provides the environment and acts as a catalyst for evolution of matter (enabling fusion, chemical reactions, etc.) while the interaction also enables the soul to co-evolve with acquired matter (constituent particles of the soul are correlated with, and will mirror, acquired matter to some degree).

\subsection{Gravitational well (spiritual well)}

A real graviton will induce effective gravitons, forming a pressure/density gradient (or curvature) of space - a gravitational well.

Density of gravitons is inversely proportional to distance, therefore, at full 
capacity the gradient of density will be proportional to gradient of orbital angular velocities of coupled bodies. These are then Keplerian velocities.

Being coupled with space, this matter is at rest relative to that space and it doesn't lose energy while orbiting.

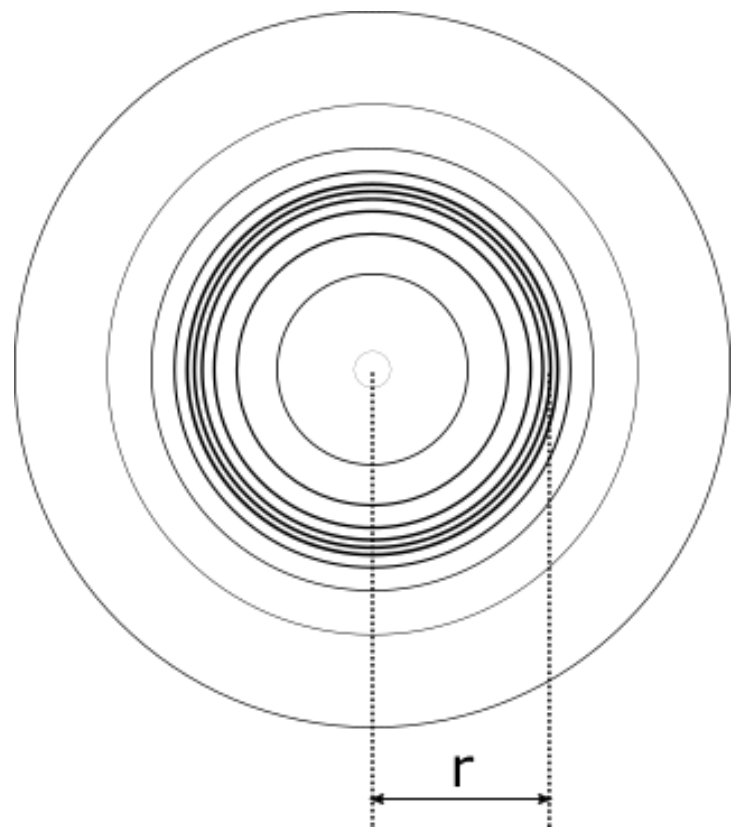

Figure 3: Gravitational well scheme

Fig. 3 shows a cross-section of a gravitational well with a [gravitational] maximum at radius r. Density of gravitons is represented by concentration of circles, it is greatest at event horizon $r$.

Graviton orbitals will, however, generally have some finite eccentricity.

Note that for a hollow sphere of standard matter, gravity is cancelled at any point inside the sphere (Shell theorem). This is generally not the case for gravity inside a hollow sphere of a naked graviton for a couple of reasons:

- gravitational potential is not absolutely the same everywhere on the sphere,

- polarization of the sphere is always greater than absolute 0 and the sphere will have openings on poles,

- the sphere is never absolutely perfect.

Note also that the sphere of a naked graviton is only relatively hollow (it 
is devoid only of standard matter, but it is not devoid of smaller scale particles, forming space) - space is not flat below radius $r$, it is filled with induced gravitons mirroring the gradient outside of the sphere.

\subsection{Black hole}

Black hole is a region of space with escape velocity greater than the speed of light.

Note that, in CR, this region does not have a singularity at the centre, it has a ring, or torus, of relative singularity at the gravitational maximum. Therefore, some material will be able to escape at the poles.

However, even though energy can escape, its path will be more or less curved and it might be difficult to observe this from reference frames where the line of sight is aligned with the axis of rotation of the black hole.

More charged a graviton is, more two-dimensional it will be and the density of the gravitational field will be decreasing from equator to the pole. Thus, the gravitational escape velocity (without taking rotation into account) can be significantly lower at the poles.

Note that, otherwise, the particles forming magnetic field lines cannot be standard photons or of standard photon rest scale, but of even smaller scale, as they would have to be faster than standard light, unless the lines are not closed.

However, generally, just as an $\mathrm{U}_{-1}$ graviton slows down from $\mathrm{c}$ with momentum transformation synchronized with coupling, a particle faster than c can similarly be slowed down and transformed to a standard photon.

This restricts the feeding potential of a black hole, thus, instead of being trapped, some matter may simply be accelerated at the equator ring and ejected through the poles at extreme velocities. In an extremely polarized case, such black hole does not acquire additional energy and is simply the most efficient transformer of energy (life-form) - transforming composite energy into individual charged particles so these can be digested elsewhere (ie. in young stars, where they combine to form hydrogen fuel).

However, in neutral black holes, most matter will have a momentum parallel to the equator plane forming a disc of orbiting material. 
The shape of a graviton explains not only the formation of jets in black holes but also why some black holes don't have them (such black holes should have a more neutral, 3-dimensional form).

The jest are not accelerated by gravity alone, the more energy there is in plasma (accretion disk) the more powerful will be the magnetic field which will focus incoming charged particles making the jets thicker at the exit. This correlation has been observed[7].

Note that magnetic field lines are, at some scale, jets of entangled particles. It is then obvious that extremely neutral bodies will have extremely weak magnetic fields, while extremely polarized will not only have extremely strong magnetic fields but will also be emitting jets of particles of larger scale (like protons and electrons, in case of black holes).

If black holes evolved before stars the farthest and biggest black holes may be more polarized. However, polarization should also be cyclic at some timescale.

Since the particles of first adjacent discrete vertical energy level are charged (dominant energy in standard particles is electro-magnetic), real gravitons of stars and planets most likely start their evolution (synchronized with coupling to standard matter) more polarized, even though most polarization may be lost already during graviton birth on that scale (inflation from smaller scale or deflation from larger scale).

Discs of material around stars and planets are thus probably formed due to charge of the host at the time of formation or at times of energy level changes - greater charge will create thinner discs. This also implies that oldest orbiting formations will orbit in a plane aligned with the plane of primordial equator of the host (unless the orbits have been disturbed later, however, probability for significant disturbance should be low after birth). Settling in equilibrium state is likely to be oscillatory and this is in Solar System confirmed with sinusoidal distribution of inclinations of planets.

Note that information on formation should be preserved in inclinations - in case the system inflated from smaller scale, nearer orbits should generally be more aligned with the equatorial plane.

This is apparently the case with the Solar System, as shown in Table 1. In case of systems deflated from larger scale, it is the farther orbits that should be more aligned. However, if vertical energy levels are discrete (as hypothesized), it is possible (or even likely) that initial energy of inflation was larger than required for the jump and the system was, after initial inflation, somewhat deflated to a stable state.

Indeed, if outer planets are bigger (and older) and the Solar System was inflating as a system of particles (ie. an atom) - which is a most likely scenario, the energy distribution suggests initial inflation of the core to current Mars' orbit or beyond, then deflation to current Sun' radius. In that case, the invariable plane (which is roughly aligned with Jupiter) might represent the fossil of the original core equatorial plane.

The higher inclinations of dwarf planets (marked green in Table 1) might indicate these are youngest and were formed after system stabilization, however, more likely, as decreasing inclination (relative to invariable plane) of dwarf plan- 


\begin{tabular}{l|l|l|l} 
Body & Inclination (ecliptic) $\left[^{\circ}\right]$ & $\begin{array}{l}\text { Inclination } \\
\text { tor })\left[{ }^{\circ}\right]\end{array}$ & $\begin{array}{l}\text { Inclination } \\
\text { plane) }\left[{ }^{\circ}\right]\end{array}$ \\
\hline Mercury & 7.01 & 3.38 & 6.34 \\
Venus & 3.39 & 3.86 & 2.19 \\
Earth & 0 & 7.25 & 1.57 \\
Mars & 1.85 & 5.65 & 1.67 \\
Vesta & 7.14 & 3.48 & 7.13 \\
Ceres & 10.59 & 3.40 & 9.20 \\
Pallas & 34.93 & 36.45 & 34.21 \\
Hygiea & 3.83 & 10.79 & - \\
Jupiter & 1.31 & 6.09 & 0.32 \\
Saturn & 2.49 & 5.50 & 0.93 \\
Uranus & 0.77 & 6.48 & 1.02 \\
Neptune & 1.77 & 6.43 & 0.72 \\
Pluto & 17.14 & 11.86 & 15.55
\end{tabular}

Table 1: Osculating orbital inclinations in the Solar System

ets in the main asteroid belt (and increasing alignment with current equatorial plane) toward the core suggests, these were inflated from smaller and neutral gravitons.

I hypothesize that outer planets (gas giants) were inflated from polarized gravitons of electrons (some, or all, of which have ended up in an excited state or different generation - tau and muon mass eigenstates, possibly multiple states in superposition), dwarf planets were inflated from neutrinos, while inner planets were inflated from positively charged particles (parts of atomic nuclei). The anti-alignment of spin magnetic momenta between inner and outer planets goes in favour of this hypothesis (which is further analysed in a follow-up paper[8]).

Note that a black hole is only relatively special form of a gravitational well. Particles faster than light must exist (even if one might not be able to detect them) and every gravitational well has a relative event horizon - digesting energy of one scale and ejecting smaller scale ions which then combine to feed moons. The only difference is scale.

The self-similarity is not limited to celestial bodies - every metabolism is ionic.

Note also that the trajectory of ejected charges is bent by the magnetic field lines (tubes) and these can be considered as a form of intestines.

In $\mathrm{CR}$, there can be no absolute singularities, only relative ones. If a black hole is the result of graviton inflation or deflation, its gravitational maximum has a real radius and, if any gravitational collapse of standard matter would occur in a black hole the collapse would end at that maximum - a ring-like (or toroidal) relative singularity. The collapse of the body of matter is, however, likely relatively synchronized with a change 
in energy level of the graviton (and exchange between gravitational and electro-magnetic potential).

The graviton may collapse to smaller scale but never to a radius of absolute 0 as this would require absolutely infinite mass or angular velocity (due to conservation of momentum). Infinite momenta (energies) are never involved in such collapses.

Furthermore, collapse to smaller radius is generally coupled with increase in angular velocity and decrease of rest mass of a graviton. This will generally be reflected in acquired mass. Conservation of momentum is thus effectively replacing gravitational attraction with centrifugal repulsion at some scale.

Therefore, although acquired mass can be compacted to extremely dense forms of energy, this energy won't occupy 0 volume and will be radiated away (at whatever scale possible) until it matches the graviton scale. However, with collapse of scale, graviton might exchange spin momentum for orbital angular momentum and decouple from acquired matter. In that case, the particles of compacted matter may be considered dead as a collective and will tend to decompose, decay and spread. Nature has evolved diverse mechanisms for such decay - in some reference frames it might be observed as rapid and abiotic, in other organic and slow.

In general, distinct conscious life (by my hypotheses) of any system (collective) starts and ends with a change in discrete energy level of a graviton (or gravitons in superposition) at times of:

- conception (coupling), synchronized with graviton inflation or deflation, and

- death (decoupling), inversion of momentum with inflation or deflation (breaking entanglement with coupled matter).

Obviously, what is interpreted as conception and what as death is scale relative. For the graviton (soul), the end of life on one scale is the beginning of life on another scale (switch of context). From some reference frames, one scale may be short-lived and graviton may be observed reincarnating on a single scale - popping in and out of existence at different spaces.

\subsection{General force, strong force and strong entanglement}

Since space cannot be absolute or absolutely abstract, it has properties and energy which can be transformed.

Various combinations of spin momenta, subspaces (dimensions) of various scales (various masses of force carrying particles), enable evolution of forces of various nature. 
Complexity of these forces will be proportional to the number of possible polarized states, or degrees of freedom in polarization.

Even gravity, with intrinsic rotation taken into account, can be interpreted as a polarized force.

A neutral force here is interpreted as a force of unipolar nature (ie. nondiscriminating attraction).

With increasing complexity, one force, may evolve from the other. Complexity can be increased by strengthening entanglement (localization in some dimension of space) of two or more sources of polarized force. Strong localization can be interpreted as superposition in some scales and, if this is a superposition of mass (ie. gravitational), bigger mass of force carrier particles will reduce the range of force.

One strongly localized force is the force holding the particles of the atom nucleus together (it is even called strong force in QM). But should it be interpreted as a special force?

If gravitational sources are generally not limited to one force carrying particle (graviton of a single scale or rest mass) such force may be interpreted as localized gravity.

Of course, if it shows complex polarization (and it may, due to relative superposition of multiple sources), it is not just localized but it has evolved from neutral gravity.

Note that polarization too is relative. It may even be induced by a polarized observer.

Note also that evolution can be regressive too - reducing, instead of increasing, complexity.

If one is to unify all possible forces and represent them by a single equation, that equation cannot contain any absolute constants. It must be as variable (or evolvable) as possible.

In practical applications, however, it will generally be more usable not to generalize as much, as variability and evolution of reality localized in an dimension of space (including time) is inevitably limited.

Therefore, instead of using this for a general force (that includes all possible interpretations on all possible scales):

$$
F=*
$$

more convenient and usable expression would be the one of a relatively general force that discards forces of negligible influence on context. 
In typical local contexts a relatively general force may include electro-magnetic and gravitational terms.

Such form is also useful in the context of transformation of energy (inflation/deflation) between discrete vertical energy levels, as all terms are hypothesized to be entangled and one potential may be exchanged for the other, ie. electro-magnetic force might regress to gravitational force with inflation of energy, but also vice versa, depending on scales in question.

This is exactly what I hypothesize has happened with the inflation of energy in the observable universe.

\subsubsection{Strong entanglement}

Strong correlation (localization) of particles in a particular dimension may be hard to disturb for an observer. Due to limited resolving power, observational energy might strengthen correlation and inflate additional pairs of entangled particles.

Note that inflation of particles on one scale may be interpreted as annihilation of particles on the other.

This is the case for particles forming atomic nuclei, held together by strong force (strong entanglement), and the reason why it is generally appropriate to consider proton as an elementary particle.

However, assuming that a binding of electron to proton, due to increasing correlation of charges, localizes proton charge into a positron, in that context, the structure of a proton becomes more complex.

Since the decrease of distance between charges will result in emission of photons, the photon can be interpreted as the product of strengthening entanglement between negative and positive charge. It then must be a composite particle, a pair of particles produced with annihilation on one scale (inflation on the other).

Obviously, if two charges are fermions (having half-integer spin momenta), the photon must be a boson (full-integer spin momentum) - elementary for some observers.

The composite particles of a photon are then fermions of smaller scale (halfphotons), which may be interpreted as an electron-positron pair of a smaller scale (or, perhaps electron-proton pair).

Absorption of that photon by entangled proton-electron pair (atom) will cause [inverse] annihilation of the pair through deflation into constituent quanta of space forming the entanglement. 
Note that, with enough energy, annihilation of a photon pair can result in inflation to an electron-positron pair.

The reason why constituent particles of the photon do not annihilate (assuming they're symmetric) between emission and absorption may be the fact they're not localized in space (travelling as a wave) although they are correlated in time. However, photon components likely do oscillate (annihilate) in mass [generation] exchanging spin momentum components, they're simply constrained by the entanglement.

This can even be interpreted as oscillation of space and time, or exchange of correlation in one dimension for the correlation in other.

Note also that the structure of emitted quanta with changes in entanglement is not limited to scaled electron/positron pairs, other particle/antiparticle combinations are possible, resulting in heavy photons or even [pairs of] neutrinos, although, in that case, these might not be interpreted as standard photons or carriers of electro-magnetic force.

Rather than transformation of spin momenta and decoupling from the entangled pair of charged particles (atom), emission of a photon can also be interpreted as radius inflation of the orbital momentum of entangled quanta of space, with average inflation velocity equal to the speed of light. In that case, the orbital momentum would only collapse (localize) to a spin momentum with absorption by another atom (pair of charged particles).

Note that orbital momentum of quanta of space might be volumetric when they are not coupled to matter, thus, they might be interpreted as thin rotating discs or hollow spheres (clouds) of space (non-localized spin momentum) at the orbital radius. The expansion of that radius is thinning the sphere surface so total volume (energy) remains relatively constant. At some scale energy will, however, be lost, and that loss will be correlated with distance or time between emission and absorption. The loss of energy of a photon, in case of the spherical expansion [propagation], may also be interpreted as caused by increasing distance in correlation between the quanta of space forming the sphere [surface]. In that case, most energy might be lost (left unabsorbed) at time of absorption, rather than during expansion.

Note, however, that loss of energy will still be correlated with distance.

Regardless of physical manifestation of photon propagation, it obviously carries information on the location of the source (which may be locally manifested as recoil in specific direction at time of absorption).

However, if correlation is physical at some scale, this direction was, at some scale, known even before the absorption (absorbers and emitters of the same 
frequencies of energy are obviously well correlated). At time of photon emission, this correlation is changed (either strengthened or weakened) and since propagation of these changes travels proportionally to distance in space it can travel faster than standard light. The potential absorber at some scale will thus be aware that photon is emitted before it arrives (it may even be interpreted as photon emission at that scale, although, the two are obviously of different scale) and integrating correlation with possible absorbers the potential absorber will, theoretically at least, be able to even calculate the probability of absorption in particular space/time.

If the potential absorber is oscillating in scale, assuming some information is conserved between scales, this allows precognition. The potential observer is even likely to interpret the information as emission of a standard photon (universes are self-similar and transfer of information between scales is also transformation of [scale] of information).

If the soul (graviton) carries consciousness and it oscillates in scale periodically, precognition or intuition should be a normal phenomenon, although amount of preserved information and proper interpretation will certainly depend on the level of evolution.

Interestingly, considering there is no visual stimulation of standard scale during deep sleep, dreams might just represent information transferred from distance and locally interpreted as absorbed photons, even if partially. Due to obvious correlation with a distant soul, in case precision of interpretation is high, dreamed event might strongly resemble past or future of the local soul's experience.

However, species concerned with short-term interests are unlikely to evolve visions of past or future although they might develop strong even if fuzzy localized intuition.

Constrained spinStrength of effective attraction between correlated particles is generally higher if their properties (momenta) are anti-aligned.

If the absorption of a photon is increasing distance between two entangled charges, the constituent quanta of the photon must then carry equal spin momentum or equal orbital momentum, depending on interpretation of absorption. If absorption of a photon is interpreted as expansion (creation) of space between two charges it is the orbital momenta that should be equal. On the other hand, if the constituent quanta are absorbed by entangled charges, it is the spin momenta that should be equal.

This may be interpreted as expansion (creation) of time between two charges if their magnetic field tubes are interpreted as time forming subspaces. 
It is reasonable to assume that the former is the correct interpretation in case of the force of electric charge while the latter is the proper interpretation in case of entanglement of magnetic spin momenta.

If constituent photon orbital momenta cannot cancel, repulsive electric force between like charges must be fictitious - decreasing distance between them is simply inversely proportional to the strength of entanglement between opposite charges that would result in increase of that distance. In that interpretation, electric force reduces to gravitational force at some scale (dimension), ie. in specific magnetic field tubes connecting opposite charges.

\subsection{Weak entanglement}

Graviton tubes are always physical, however, with increasing spatial distance and no additional energy applied to the tube, physical entanglement is weak.

However, as long as there is no change in entangled energy the entanglement will not get broken (and when it does, it never is broken absolutely), even if the particles are spatially separated over great distance.

Due to the fact that energy remains constant, either volume and energy density of the tube (dimension) connecting the particles remain constant or energy density is decreased proportionally to volume increase. Assuming information is transferred along the tube membrane, the former will be, with decreasing tube radius (and mass per quantum of space [cross-section]), increasing speed of information transfer. In that interpretation, speed of transfer can exceed speed of light. In the second interpretation, space is stretched and local observer (within the tube) would measure no change in speed, while remote observer could measure superluminal transfer - assuming tube (subspace) is resolvable by that observer.

In $\mathrm{CR}$, both interpretations are valid (possible). One can only argue what and when any information is transmitted. Information that is observable could be limited to information transmitted with the collapse (deflation) of entanglement, which will, in both interpretations, decrease energy in the tube.

Since weak entanglement is relatively unaffected by change in spatial distance, the first interpretation (volume invariant to spatial distance) is convenient as it implies proportionality of volume (or volumetric distance) with distance in correlation (inverse of [scale of] entangled energy or strength of entanglement).

Note that strength of entanglement is relative. Observation can both strengthen and weaken the entanglements - the outcome will generally depend on spatial distance and equilibrium strength.

Note also that the observer who cannot resolve (observe) the tube of entanglement cannot use the tube for information transfer or control information transmitted on collapse[9] (superluminal or not). In that 
case, weak entanglement reduces to standard non-local QM entanglement (note that, per CR postulates, QM entanglement cannot be absolutely non-local, implying existence of reference frames with interpretations stated above).

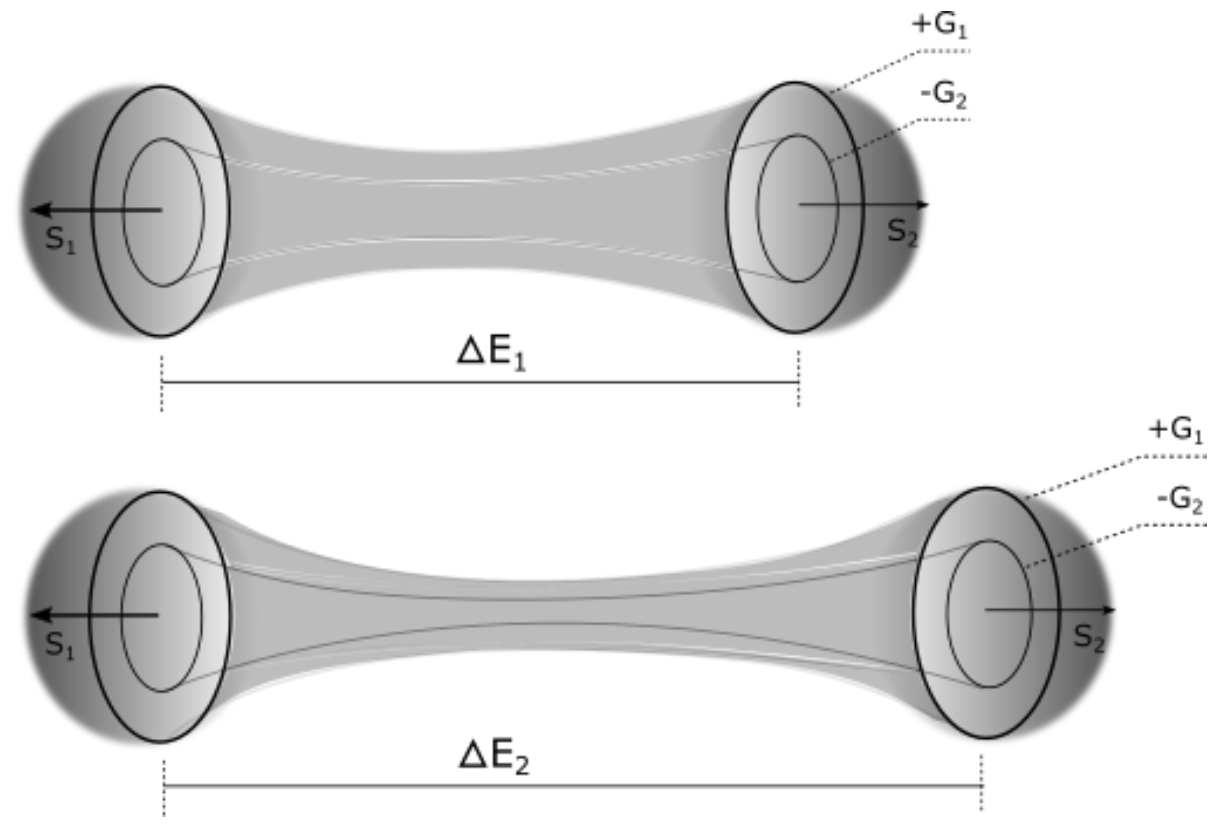

Figure 4: Weak entanglement

$$
\Delta E_{1}=\Delta E_{2}=\Delta E=\text { const. }
$$

\subsection{Electric polarization}

In the standard model of physics particles have fixed (intrinsic and unchangeable) properties (ie. electric charge, rest mass, spin) which then produces a zoo of different particles. While that approach is useful, different particles evolve from other particles and it might not always be the most convenient approach, especially in CR, where even planets or animals on it are considered particles from certain reference frames.

One can thus model a single particle and declare it relative - evolvable. Here, one such particle is the graviton - it can transform from a source of gravity to a source of electro-magnetic force and vice versa (effectively exchanging the scale of mass with the scale of charge). 
In example, down quark could evolve from a superposition of 9 gravitons of electron mass, where 5 of them are negatively charged, 4 positively, and $2 / 3$ of remaining negative charge (4 opposite charges cancel) have been exchanged for mass.

This gives a total electric charge of $-1 / 3$ e, spin $1 / 2$ and rest mass (assuming quadratic growth of mass) equal to:

$$
m_{d 0}=\left(9+\left(\frac{2}{3}\right)^{2}\right) m_{e}=\left(3^{2}+\left(\frac{2}{3}\right)^{2}\right) * 0.511 \frac{M e V}{c^{2}}=4.826 \overline{1} \frac{M e V}{c^{2}}
$$

However, assuming this superposition is stable (particles have undergone fusion and indeed form a new particle), total mass will be somewhat lower (by the binding energy).

Binding energy is not always lower though, for bound hadrons (ie. quarks bound into a proton), due to large angular momenta, total mass can be significantly higher than the sum of rest masses of bound particles (from some reference frames).

Assuming particles are bound as atomic nuclei are, and scaling binding energy of ${ }^{9} \mathrm{C}$ (carbon isotope), the rest mass becomes:

$$
m_{d}=m_{d 0} \frac{M e V}{c^{2}}(1-0.00464)=4.8037 \frac{\mathrm{MeV}}{c^{2}}
$$

This is in good agreement with mass determined from lattice QCD[10] of $4.79 \pm 0.16$ $\mathrm{MeV} / \mathrm{c}^{2}$.

However, here it was assumed that complete conversion of 1 e of charge would result in mass equal to electron mass, the actual ratio can be significantly different. Assuming that the down quark is instead the result of conversion of anti-up quark charge to mass, mass inflated per $1 / 3$ e of charge lost is:

$$
\left(m_{d}-m_{u}\right)=2.78 \frac{\mathrm{MeV}}{c^{2}}
$$

Note that conversion of electric charge to gravitational mass by that ratio would release enormous amounts of energy as gravitational force is $\approx 10^{42}$ orders of magnitude weaker than electro-magnetic at that scale. Instead of energy released, it may be more appropriate to consider conversion of electro-magnetic force to strong nuclear force (as quarks are confined to atomic nuclei).

Strong nuclear force then evolves from electro-magnetic force, however, it may be interpreted as condensed gravitational force. During the conversion, instead of inflation of rest mass, local space is condensed, the mass of local gravity carrier particles increases, strongly limiting the range of the force (localizing gravity). 
Of course, if the strong force is polarized, it is a composite of different sources and thus more complex.

Symmetric positive and negative charges in superposition can annihilate and produce more massive particles (such as quarks) with enough kinetic energy. Thus, taking kinetic energy into account, quarks (or, more precisely, quark/antiquark pairs) can be produced with only 2 charges and that is how they might be generally produced in the observable universe at this time.

Electric polarization of a graviton is represented by positive $(+)$ or negative (-) charge. As such, they are sources of electric, magnetic fields and electro-magnetic radiation. In CR, positively charged particles may be referred to as anti-matter, negative as matter. Composite, neutral, matter particles are those with more mass in negative charge, neutral anti-matter particles are those with more mass in positive charge.

In $\mathrm{CR}$, there is no missing anti-matter problem.

Consider elementary charges (electrons and constituent particles of protons and neutrons), as shown in Table 2. Obviously, charges are balanced, all parti-

\begin{tabular}{l|c|r} 
particle & charge quanta $[\mathrm{e}]$ & total charge $[\mathrm{e}]$ \\
\hline electron & $-2 / 3-2 / 3+1 / 3 ?$ & -1 \\
proton & $+2 / 3+2 / 3-1 / 3$ & +1 \\
neutron & $+2 / 3-1 / 3-1 / 3$ & 0
\end{tabular}

Table 2: Elementary charges

cles are fermions and assuming positive charges are anti-matter particles, negative are matter particles, the result of asymmetry is simply exchange between charge and mass. In CR, electron cannot be absolutely elementary, therefore, three down quarks $(3 *-1 / 3$ e) and three up quarks $(3 *+2 / 3$ e) could have evolved from 1 electron and 2 positrons.

All the elementary particles could have thus evolved from 2 electrons and 2 positrons total - symmetry, assuming that for every electron there exists one proton and one neutron.

Having equal amount of charge, but (orders of magnitude) different mass, electrons and protons could be interpreted as belonging to two different universes.

Quarks cannot be absolutely elementary either. For every electron there is thus a positron hiding beyond the relative event horizon of an atom - it may be reformed (from proton energy) at time the electron is captured by the proton to form the atom (leaving $+1 / 3$ e and $-1 / 3$ e charge with neutral mass in the core).

In one interpretation, proton itself is the positron evolved in time in order to preserve existence (avoid annihilation), one may refer to it as a vertically 
excited positron (conventional anti-proton would then be a vertically excited electron).

Existence requires asymmetry, and with asymmetry evolves diversity.

In one interpretation of $\beta^{-}$decay, a quantized collapse of a gravitational maximum (neutron event horizon) produces a positron/electron pair and neutrino/antineutrino pair, electron and anti-neutrino are ejected (paired or evolved into a $\mathrm{W}^{-}$boson initially by the strong curvature) while positron and neutrino are absorbed by the nucleus.

Strong nuclear curvature evolves positron/neutrino pair into up quark $(+2 / 3$ e) and anti-down quark $(+1 / 3$ e) which then annihilates with one existing down quark $(-1 / 3$ e), transforming neutron to proton.

Outside the strong curvature (pressure) of space of nuclei, $\mathrm{W}^{-}$boson is unstable and lack of gravitational pressure (time dilation) may be the sole reason it decomposes back to electron/anti-neutrino pair.

The Compton wavelength of the electron is on the order of atomic radii and this suggests that electron bound to the atom is electron coupled to a nuclear graviton of similar mass (and thus similar range). Electrons closer to the nucleus are then coupled with more massive gravitons. One can then consider the up quark as a positron coupled with such graviton and a down quark as electron coupled with an even more massive graviton. Note that, in these couplings, mass is inversely proportional to charge, suggesting mass/charge exchange with coupling.

Coupling of an electron and anti-neutrino into a massive $\mathrm{W}^{-}$boson then suggests that electric charge of a massive $\mathrm{W}$ boson may be extremely low ( $1 \mathrm{e}$ ) - in any case, charge is not conserved in that form, it is restored with decoupling.

The charge/mass exchange can be understood as exchange of spin momentum components. To conserve momentum, with increasing rest mass (or spin momentum of mass) the momentum of energy producing charge (magnetic field lines) must be decreased. If one interprets magnetic field lines (tubes) as dimensions of time, time dilation is physically manifested here as contraction or deflation of time dimensions.

Disregarding negligible photon mass compared to a strong force graviton mass, conversion of $2 / 3$ e charge potential to gravitational Yukawa type potential of a down quark mass would yield mass $\mathrm{m}_{g}$ for the graviton (assuming unscaled gravitational constant $\mathrm{G}$ ) as follows:

$$
\begin{gathered}
\frac{G M}{r} e^{-\mu_{g} r}=\frac{2}{3} \frac{1}{4 \pi \epsilon_{0}} \frac{q_{e}}{r} \\
e^{-\mu_{g} r}=\frac{2}{3} \frac{q_{e}}{4 \pi \epsilon_{0}} \frac{1}{G M} \\
\mu_{g}=\ln \left(\frac{2}{3} \frac{q_{e}}{4 \pi \epsilon_{0}} \frac{1}{G M}\right) \frac{1}{r} \\
m_{g}=\ln \left(\frac{2}{3} \frac{q_{e}}{4 \pi \epsilon_{0}} \frac{1}{G M}\right) \frac{1}{r} \frac{\hbar}{c}
\end{gathered}
$$




$$
\begin{array}{r}
\mathrm{q}_{e}=1.60218 * 10^{-19} \mathrm{C} \\
\epsilon_{0}=8.85419 * 10^{-12} \mathrm{~F} / \mathrm{m} \\
\mathrm{G}=6.67430 * 10^{-11} \mathrm{~m}^{3} \mathrm{~kg}^{-1} \mathrm{~s}^{-2} \\
\hbar=1.054572 * 10^{-34} \mathrm{Js} \\
\mathrm{c}=2.99792458 * 10^{8} \mathrm{~m} / \mathrm{s}
\end{array}
$$

For $\mathrm{r}=1 * 10^{-15} \mathrm{~m}$ and mass of a down quark $\mathrm{M}=\mathrm{m}_{d}=4.8 \mathrm{MeV} / \mathrm{c}^{2}=$ $8.556777 * 10^{-30} \mathrm{~kg}$ :

$$
m_{g}=2.44819 * 10^{-26} \mathrm{~kg}=13.7333 \frac{\mathrm{GeV}}{\mathrm{c}^{2}}
$$

Roughly 100 times the range $(\mathrm{r})$ would give the mass of a pion ( $\pi$ meson). It is certainly viable that the range of gravity of the down quark is 100 times the nuclear radius, at least when there's an electron bound to the nucleus (forming the atom).

Of course, the source of a strong force is generally a composite force (a superposition) of multiple short range sources, but is the complex polarization present at all times, or does it only occasionally evolve?

Interestingly, using Compton wavelength of a pion (roughly $1.43 * 10^{-15}$ m) for $\mathrm{r}$, gives $\mathrm{m}_{g}=9.60 \mathrm{GeV} / \mathrm{c}^{2}$, which would be equal to superposition (not a stable composite) of 2 bottom quarks and 1 charm quark $(2 * 4.18+1.28=$ $9.64 \mathrm{GeV} / \mathrm{c}^{2}$ ). Such superposition could be interpreted as an unstable neutral baryon. If that baryon is then paired with its anti-particle the radius $\mathrm{r}$ would be reduced to roughly $0.715^{*} 10^{-15} \mathrm{~m}$, which could be interpreted as down quark contribution to mass radius of the proton.

Note that the calculation for up quarks (conversion of $1 / 3$ charge to mass) gives almost equal results (due to up quark mass being roughly half the down quark mass, $2 / 3 \mathrm{~m}_{d}^{-1} \approx 1 / 3 \mathrm{~m}_{u}{ }^{-1}$ ).

Note also that it has recently been discovered that a charm/anticharm quark pair may be more intrinsic to the proton than previously thought[11]. Could it be that the whole hypothesized baryon/antibaryon pair gets periodically inflated then?

If one assumes that the mass of the pair is inflated by the ratio of charm quark mass to proton mass $(1275 / 928.272)$, the mass radius becomes $0.521 * 10^{-15} \mathrm{~m}$, in agreement with recently obtained proton mass radius of $0.55 \pm 0.03 \mathrm{fm}[12]$.

Thus, the nuclei of atoms may generally not be held together by a force stronger than electrostatic repulsion, rather the repulsive electric potential is periodically converted to strongly localized gravitational potential. This oscillation could be interpreted as relative superposition of electro-magnetic and gravitational forces, which collapses to a particular eigenstate with interaction (observation). 
The requirement for nuclear fusion (fossilization of superposition of multiple atomic nuclei into a new discrete nucleus) is then anti-aligned oscillation. The binding will be most stable with a phase shift of $90^{\circ}$, while it is least stable in resonance (stability of superposition in that case requires extremely low pressure/temperature).

Note that, in CR, absolutely infinite stability is impossible. Therefore, any superposition is a relative fusion, and vice versa.

This difference in phase shift can be achieved with difference in [the amount of] momenta between two nuclei (inducing time dilation in one) - bombardment of one nucleus with the other.

This suggests that the probability for fusion may be higher between different species (different rest masses between nuclei).

Interestingly, the non-dimensional factor in equation (1.3) is almost equal in value to the natural logarithm of the inverse of electron mass $\left(\mathrm{m}_{e}\right)$ :

$$
\ln \left(\frac{2}{3} \frac{q_{e}}{4 \pi \epsilon_{0}} \frac{1}{G M}\right) \approx \ln \left(\frac{1}{m_{e}}\right)
$$

Rearranging (1.3), one can get the orbital angular momentum of the graviton (in local rest frame). Assuming inverse of $2 / 3$ of electron mass (better fit):

$$
\begin{aligned}
& m_{g} c r=\ln \left(\frac{3}{2} \frac{C}{m_{e}}\right) \hbar \approx \ln \left(\frac{3}{2} \frac{1}{m_{e}}\right) \hbar \approx \sqrt{69 *(69+1)} \hbar \\
& \qquad \mathrm{C} \approx 1 \mathrm{~kg}
\end{aligned}
$$

Is it a coincidence that graviton momentum is quantized by [the inverse of] $2 / 3$ electron mass?

Here, one should also question whether the $\hbar$ constant (quantum of momentum) should be different (scaled) in the local rest frame.

Down quark mass $\left(\mathrm{m}_{d}\right)$ from the equivalence:

$$
\frac{2}{3} \frac{q_{e}}{4 \pi \epsilon_{0}} \frac{1}{G M}=\frac{3}{2} \frac{1}{m_{e}}
$$

is:

$$
\begin{array}{r}
m_{d}=M=\left(\frac{2}{3}\right)^{2} \frac{q_{e}}{4 \pi \epsilon_{0}} \frac{m_{e}}{G}=8.7348 * 10^{-30} \mathrm{~kg}=4.9 \frac{\mathrm{MeV}}{c^{2}} \\
\mathrm{~m}_{e}=9.10938356^{*} 10^{-31} \mathrm{~kg}
\end{array}
$$


The obtained mass is in agreement with lattice QCD $\left(4.79 \pm 0.16 \mathrm{MeV} / \mathrm{c}^{2}\right)$. Thus, the constant $\mathrm{C}$ here may be exactly equal to 1 , rather than approximately.

The obtained equation (1.4) also confirms the $(2 / 3)^{2}$ factor of charge/mass conversion, initially assumed in (1.2).

Some might argue that graviton orbital velocity cannot be equal to c as it has finite mass, however, orbital momentum of the graviton should be understood as orbital momentum of [a quantum of] space - it is relatively massless from the local reference frame.

However, once it gets coupled its orbital velocity will decrease below c (and mass will inflate to conserve the momentum). The coupling (inflation) of a graviton will decrease the local gravitational capacity for coupling with a particular scale of matter, however, as this coupling is also a gravity source, it may increase coupling capacity of the local well for another scale of matter.

\subsection{Mass}

Mass is simply energy, or relatively concentrated energy in some contexts. No quantum of energy can have mass equal to absolute 0 . A particle with a relative 0 mass should be understood as entangled non-concentrated finite mass $(>0)$, generally in the form of waves.

\subsection{Total mass (gravitational mass)}

Uncoupled graviton may be considered to have 0 mass relative to space. However, once coupled (and slowed down) its mass is greater than 0 .

Total mass is the sum of mass of a graviton (img mass) and mass of acquired (coupled) matter (real mass):

$$
M=m_{i m g}+m_{r e}
$$

It is usually denoted with uppercase letter M. In contexts where it represents a quantum of bigger mass, it may be denoted with lowercase letter $\mathrm{m}$.

\subsection{Imaginary mass (virtual mass, img mass)}

Imaginary mass is the mass of a coupled graviton. However, in one interpretation, some or all of this mass may be shielded by acquired (coupled) matter and in that case it is not constant (although acquisition of matter to full capacity can be relatively instant).

It can then be interpreted as the unshielded mass of a graviton and it is a relative 0 in equilibrium (full capacity). If there is no shielding, at full capacity img mass is equal to coupled real mass. 
The mass of a coupled graviton is usually denoted with $\mathrm{m}_{i m g}$.

Note that if shielding is real, total mass is constant as long as the well is not over-capacitated.

\subsection{Real mass}

A naked graviton will attract matter. Real mass represents the acquired mass (mass coupled to the graviton).

However, coupling is relative to scale. In example, standard matter forming Earth's body and atmosphere is assumed to be coupled with a graviton of similar scale as the sum of that matter (I hypothesize radius equal to Earth's inner core radius). This coupling is, however, also quantized into smaller couplings. The smaller scale gravitons forming space of the large scale graviton (or, space of the Earth), are coupled to atoms.

Note that in case of mass shielding, at full capacity large graviton effectively doesn't exist.

The Moon is also relatively coupled to this graviton, however, density of space decreases with distance and the number of coupled small scale gravitons is lower.

Note that Earth is similarly coupled to the Moon's graviton.

However, large scale graviton equivalent to the sum of matter [held together by mutual attraction] may not always be present. This is assumed to be the case in life-forms considered dead.

Asteroids and comets are assumed to be such bodies. These are most likely leftovers of dead planets and moons. However, it is possible that every barycentre of organized or localized mass has a physical interpretation in the form of a more energetic graviton (compared to constituent gravitons of that mass), although this is probably unlikely and even if true, distinct consciousness of such body [as a collective] would be extremely low (a relative 0 ).

One might argue that large scale graviton is unnecessary in any case, however, recursion would then make gravitons of any scale unnecessary 
and there would be no gravity or any other force at any scale (note that a small scale graviton coupled to an atom is a large scale graviton from the reference scale of atoms).

Real mass is usually denoted with $\mathrm{m}_{\text {re }}$.

If velocity of a graviton is different than its rest velocity in that space, kinetic energy will be stored in the graviton or constituent quanta of its own space (relativistic effects are at least partially real in this case) as img mass.

The increase in gravitational potential will then result in the increase of real mass if matter of appropriate scale is available in local space.

Real mass in a distinct gravitational well [of a graviton] is generally in significant part always being transformed to other forms of energy (through fusion, heat, chemical reactions, etc.) and lost energy will generally be periodically replenished, as long as real mass is available (effectively, in case of shielding interpretation, imaginary mass is periodically being exchanged with real mass).

Note that continuous existence of [oscillating] transformation of energy may generally signal the existence of a discrete living being (superposition of a collective reflected in relative mental singularity - distinct consciousness).

Universes are self-similar, and with recursion, any acquired real mass (or matter) of scale $\mathrm{n}$ is total mass of another, generally smaller, scale:

$$
\sum m_{r e}(n)=\sum M(n-1)
$$

At full capacity, angular velocity of space (effective gravitational field tube) at radius $r$ of a gravitational well is defined by this equation (derived from Kepler's laws), with the assumption of a point-like source of gravity:

$$
v_{s}^{2}=r g=\frac{G M}{r}
$$

Here, $\mathrm{M}$ is the total mass of the gravity source (or mass below radius $\mathrm{r}$ ) and $\mathrm{G}$ is the gravitational constant.

while mass capacity of the well is:

$$
C=M-m_{r e}=m_{i m g}
$$


Well capacity is related to spin velocity of its graviton (at its mass radius $r_{s}$ ), which at full capacity is Keplerian:

$$
c_{s}^{2}=r_{s} g_{s}=\frac{G M}{r_{s}}=G \frac{m_{i m g}+m_{r e}}{r_{s}}=2 G \frac{m_{i m g}}{r_{s}}
$$

However, evidently, angular velocities in gravitational wells are not always Keplerian (ie. dark matter problem, spin momenta of planets).

Such velocities should then indicate either under-capacitated or over-capacitated gravitational wells. In case of excess velocity, the well is under-capacitated (imaginary mass is greater than coupled real mass).

Note that gravitons are generally orbiting at the speed of light (local speed limit), they are only slowing down with coupling, reaching Keplerian velocity at full capacity.

In the process, they are inflating - exchanging orbital angular momentum for spin momentum.

In case of lack of velocity, the well is over-capacitated (real mass is greater than img mass).

Effectively, in under-capacitated wells space is dragging matter, in overcapacitated wells matter is dragging space.

Assuming thus that coupled mass has Keplerian velocity at full capacity on particular orbital radius (note that particular orbital radius represents the range of coupling gravitons), conservation of momentum dictates that for unfilled capacity $\left(\mathrm{m}_{r e}<\mathrm{m}_{i m g}\right)$ at the same radius velocity must increase.

Decoupling can be simply achieved with the inversion of a spin of a graviton (ie. with collapse of entanglement), which is generally synchronized with a change in scale, even if that change may be temporary.

If real mass coupled to a graviton (gravitational maximum) is compact and forms a solid-like body (period of rotation is constant and doesn't depend on distance from the graviton) with isotropic energy distribution then it can be considered as a point particle rotating about the barycentre of mass (also centre of graviton). If momentum of that mass is lower than Keplerian velocity at the mass [orbital] radius (the well is over-capacitated), its motion is relativistic in that well. Proper (or properly scaled) relativistic equation for total mass is then:

$$
M=m_{r e}+m_{i m g}=\frac{m_{r e_{0}}}{\sqrt{1-\frac{v_{r e} e^{2}}{c_{s}^{2}}}}+m_{i m g}
$$




$$
\begin{gathered}
m_{r e_{0}}=\left(M-m_{i m g}\right) \sqrt{1-\frac{v_{r e}{ }^{2}}{c_{s}{ }^{2}}} \\
v_{r e}=\frac{2 \pi r_{r e}}{T_{r e}} \approx \frac{2 \pi r_{s}}{T_{r e}}
\end{gathered}
$$

$\mathrm{v}_{r e}, \mathrm{r}_{r e}, \mathrm{~T}_{r e}=$ orbital velocity, radius and period of rotation of real mass, respectively $\mathrm{c}_{s}, \mathrm{r}_{s}=$ Keplerian angular velocity and mass radius of the maximum, respectively

Here, of course, $\mathrm{v}_{r e}$ and $\mathrm{T}_{r e}$ should be relative to rest velocity (expected Keplerian velocity) and period when calculating local excess energy.

Note that spin angular velocity of [real mass of] Earth is lower than Keplerian velocity. Earth's spin is thus locally relativistic and the gravitational well has excess energy. In one interpretation that is the reason the planet is active - transforming, or digesting, energy (through fission, heat, chemical reactions, etc.). In another interpretation, it is these transformations (particularly thermal energy) that are converting orbital angular momentum to radial. Cause and effect are relative in $\mathrm{CR}$, and both interpretations are valid.

If real mass is quantized into multiple bodies with different periods of rotation, mass equation is:

$$
M=\sum \frac{m_{r e_{0}}}{\sqrt{1-\frac{v_{r e}{ }^{2}}{c_{s}}}}+m_{i m g}
$$

\subsubsection{Predicting activity (amount of local life)}

Since locally relativistic motion is correlated with local energy transformation, predictions can be made about local activity. The more excess energy there is, the higher is the probability for complex transformations and thus complex life.

Mass radius of terrestrial bodies should be the radius of a gravitational maximum (major, or large scale graviton), with the assumption that terrestrial bodies are solid or solid-like and that the mass radius is the radius of the core (inner core, if it exists), excess energy can be easily calculated.

For terrestrial bodies:

$$
\begin{gathered}
M=\frac{m_{r e_{0}}}{\sqrt{1-\frac{v_{r e} e^{2}}{c_{s}^{2}}}}+m_{i m g}=\frac{m_{i m g}}{\sqrt{1-\frac{v_{r e}^{2}}{c_{s}^{2}}}}+m_{i m g}=m_{i m g}\left(1+\frac{1}{\sqrt{1-\frac{v_{r e}^{2}}{c_{s}^{2}}}}\right) \\
v_{r e}=\frac{2 \pi r_{r e}}{T_{r e}}=\frac{2 \pi r_{s}}{T_{0}}-\frac{2 \pi r_{s}}{T}=c_{s}-\frac{2 \pi r_{s}}{T}
\end{gathered}
$$




$$
\begin{aligned}
T_{0} & =\frac{2 \pi r_{s}}{c_{s}} \\
c_{s}^{2} & =\frac{G M}{r_{s}} \quad \mathrm{G}=6.674 * 10^{-11} \mathrm{~m}^{3} \mathrm{~kg}^{-1} \mathrm{~s}^{-2}
\end{aligned}
$$

\begin{tabular}{|c|c|c|c|c|c|c|c|c|}
\hline body & $\begin{array}{l}\text { total } \\
\text { mass } \mathrm{M} \\
{\left[10^{24}\right.} \\
\mathrm{kg}]\end{array}$ & $\begin{array}{l}\text { img mass } \\
\mathrm{m}_{i m g} \\
{\left[10^{21} \mathrm{~kg}\right]}\end{array}$ & $\begin{array}{lr}\text { mass } & \text { ra- } \\
\text { dius } & \mathrm{r}_{s} \\
{[\mathrm{~m}]} & \end{array}$ & $\begin{array}{l}\text { rotation } \\
\text { period } \mathrm{T} \\
{[\mathrm{h}]}\end{array}$ & $\begin{array}{l}\text { relativistio } \\
\text { angular } \\
\text { velocity } \\
\mathrm{v}_{r e} \\
{[\mathrm{~m} / \mathrm{s}]}\end{array}$ & $\begin{array}{l}\text { Keplerian } \\
\text { period } \\
\mathrm{T}_{0}[\mathrm{~h}]\end{array}$ & $\begin{array}{l}\text { Keplerian } \\
\text { velocity } \\
\mathrm{c}_{s}[\mathrm{~m} / \mathrm{s}]\end{array}$ & $\begin{array}{l}\text { excess } \\
\text { mass } \mathrm{m}_{x} \\
{\left[10^{24} \mathrm{~kg}\right]}\end{array}$ \\
\hline Mercury & 0.330 & 4.8728 & 567028 & 1407.6 & 6231.6 & 0.1588 & 6232.3 & $\begin{array}{l}0.3203 \\
(97 \%)\end{array}$ \\
\hline Venus & 4.868 & 63.6013 & 3079911 & 5832.6 & 10269.8 & 0.5234 & 10270.7 & $\begin{array}{l}4.7408 \\
(97 \%)\end{array}$ \\
\hline Earth & 5.972 & 534.1110 & 1206084 & 23.9345 & 18090.9 & 0.1158 & 18178.8 & $\begin{array}{l}4.9038 \\
(82 \%)\end{array}$ \\
\hline Mars & 0.642 & 109.8515 & 1581777 & 24.6229 & 5092.5 & 0.5304 & 5204.6 & $\begin{array}{l}0.4223 \\
(66 \%)\end{array}$ \\
\hline Pluto & 0.01303 & 0.6371 & 224993 & 153.2928 & 1963.4 & 0.1997 & 1966.0 & $\begin{array}{l}0.0118 \\
(91 \%)\end{array}$ \\
\hline
\end{tabular}

Excess mass is:

$$
m_{x}=\frac{m_{i m g}}{\sqrt{1-\frac{v_{r e} e^{2}}{c_{s}^{2}}}}-m_{i m g}
$$

Table 3: Spin momentum components and excess energy of terrestrial bodies*

* Sources of data in Table 3 for total mass and rotation period are NASA Planetary Fact Sheets[13].

Calculated values for some terrestrial bodies are shown in Table 3 (values in parentheses for excess mass are percentages of total mass). Unsurprisingly, relativistic (excess) mass percentage of total mass is, for terrestrial planets, decreasing with distance from the Sun. While Earth's excess (and thus complexity potential) is highest in absolute value, Venus has a maximum potential for complexity relative to its rest energy (its interior is probably very active). However, in all bodies relativistic energy is high, and where pressure/temperature allows it (in interior generally) one probably can expect complex life.

Complex life as we know it implies fluid-like interactions - transformation of random thermal fluctuations into organized flows of energy. This could be, however, interpreted as increase in scale of thermal energy 
and pressure. Existing models of planetary interiors are based on the assumption of accumulation of mass (and a continuous gradient of pressure/temperature) - not coupled with inflation of a small scale graviton to large scale.

In cases where there was inflation (which should generally be the case for stars, planets and dwarf planets), these models must be wrong, as gravitational maximum should generally be represented by a spherical surface of a significant radius - not a point. And if changes in energy levels are frequent, multiple matter pressure/density maxima will exist. Therefore, possibility for complex life is likely to exist in any layer of a mantle and probably for significant time even after graviton deflates and decouples from the body.

Taking asteroid bombardment and collisions into account, both, probability for complex life and maximal possible (evolvable) complexity are, with inflation hypothesis, much higher in interiors of celestial bodies.

Changes in energy level of a graviton can produce significant thermal energy, allowing complex life even at large distances from a star.

The discovered features and activity (complexity) on Pluto and Charon[14] is thus not surprising. The fact that there are no small craters on Pluto[15] should then probably be interpreted as relatively recent tectonic activity, rather than lack of smaller objects in Kuiper belt (as is the current interpretation[16]).

Note that, if changes between energy levels of gravitons are relatively frequent (on the order of millions of years or less), crater counting is not the appropriate method for estimation of surface age. With oscillation (inflation/deflation) of gravitons, not only would surfaces of bodies be periodically refreshed, asteroid bombardments should be more common and relatively periodic as long as oscillation exists.

Consider oscillation of a graviton between the $1 e$ moon and superposition with a graviton of the planet it is orbiting. Deflation of a moon graviton would result in decay of locally relativistic energy. While that decay may be relatively slow, deflation of a graviton is not instant and it may even exit the surface with a radius greater than relative 0 (and high velocity) which would cause ejection of compressed real mass toward the planet. Similarly, it may start inflating before it reaches the surface of the planet, creating a tube (exact shape would depend on graviton polarization) increasing in size and curvature toward the centre. At the same time the tube would be filled with fluid (ie. magma, water).

Due to graviton polarization (which is always present at some scale and increases with deflation in this case) the graviton is most likely to enter/exit the body at magnetic poles. These will generally be aligned with geographic south and north poles in significantly polarized bodies. However, in case of low asymmetry between momenta of oppositely charged constituent components (or presence of anti-aligned superposition), the 
most likely point of exit/entrance may be aligned with the orbital plane of the moon. In that case, possible impact latitude may range from $0^{\circ}$ to $\pm \mathrm{a}$, where a is the sum of planet's obliquity and moon's orbital inclination.

Since Earth is polarized (polarization was also likely stronger in the past), most likely location of previous entrances is the south pole. However, polarization of the Earth's current moon is low and Earth's polarization is declining (relatively chaotic and fast movement of magnetic dip poles probably also indicates pending reversal). Therefore, in case of Earth, future graviton entrance/exit latitude might be somewhere between $0^{\circ}$ to $\pm\left(23.44^{\circ}+5.14^{\circ}\right)= \pm 28.58^{\circ}$ degrees. The current obliquity to orbit of the Moon should likely be added to that range (axis of rotation of the graviton coupled to Moon's body should be aligned with the axis of rotation of the Moon while they are coupled), extending the range to $\pm 35.26^{\circ}$.

Note that large massive extinctions suggest Earth might have had more moons in the past, but it is also possible that the current moon was destroyed and reformed multiple times at various distances (albeit increasing with time as real mass, due to conservation of momentum, likely continues on tangential trajectory once uncoupled).

Note also that if graviton exit from the moon is non-polar while planetary entrance is polar, asteroids formed with exit will not impact the planet at the pole, unless they too are polarized - which is not impossible, magnetic moment of the graviton could be fossilized in ejecta.

Assuming asteroid and graviton decouple near the Moon surface in nonpolar exit and asteroid velocity exceeds escape velocity, possible range for asteroid impacts on Earth is a superposition of $0^{\circ}$ to $\pm 35.26^{\circ}$ and $0^{\circ}$ to $\pm 21.9^{\circ}$.

Interestingly, the Chicxulub crater, at $21.4^{\circ} \mathrm{N}$, is within both ranges. Also interesting is the fact that the volcanic region under ice in Antarctica (with more than 130 volcanoes[17]) is the largest on Earth.

On Mars, considering orbital inclination of its moons (or, more likely, moon remnants), Valles Marineris may be the product of impacts formed by asteroids produced by moon graviton decoupling.

Interestingly, Keplerian period $\mathrm{T}_{0}$ is similar between Venus and Mars and between Mercury and Earth. In follow-up work[8] I hypothesize that Mercury and Earth are in $2 e$ configuration (superposition of 2 gravitons), while Venus and Mars are 1e planets (single graviton).

This suggests that Keplerian periods are relatively quantized and should be roughly equal for planets in the same configuration. However, Keplerian period depends on current energy level. Similar Keplerian periods should thus be correlated with relatively equal energy levels.

Periods relatively invariant to mass go in favour of hypothesis of graviton 
inflation, as this implies proportionality between graviton mass and mass radius $\left(\mathrm{r}_{s}\right)$.

Note that mass radius for Venus and Mars is simply the core radius (as their cores apparently don't have inner and outer parts at this time).

I hypothesize that the formation of the inner core is synchronized with the compression (deflation) of a graviton to a smaller radius. In that case, outer core radius (discontinuity between the core and mantle) is a fossilized former graviton radius, while inner core radius (discontinuity between inner and outer core) represents the current graviton radius. If there is no inner core, current graviton radius is likely the outer core radius (assuming presence of the inner inner core can be excluded).

This should be interpreted as a change in energy level of a graviton. Since these are discrete, there are constraints on the radius - in this case it should change (scale) with a square root of $2^{n}$ where $\mathrm{n}$ is a positive or negative integer.

Strong discontinuities indicate that changes in energy level are relatively frequent. Therefore, most likely current energy level is the one with a most solid discontinuity (one which most closely matches the corresponding energy level).

Due to required oscillation (and frequency of energy level changes), however, discontinuities may somewhat deviate from radii of graviton energy levels in any case.

Earth's mass radius has been calculated with the assumption that Earth's graviton surface gravity is equal to gravity at the Sun's surface $\left(274 \mathrm{~m} / \mathrm{s}^{2}\right)$, from:

$$
g_{s}=\frac{G M}{r_{s}^{2}}=274 \frac{m}{s^{2}}
$$

which gives radius $\mathrm{r}_{s}$ of $1206 \mathrm{~km}$, almost equal to estimated inner core radius $(1216 \mathrm{~km})$.

Note that Earth's [dominant] charge radius is then most likely at $\sqrt{2} \mathrm{r}_{s}$, giving gravity equal to $137 \mathrm{~m} / \mathrm{s}^{2}$, which gives a g-factor of 2 , equal to electron or positron g-factor.

Since g-factor is dimensionless, it is relatively scale invariant (ie. electrons and muons have almost equal g-factors) and this implies that Earth's graviton has been inflated from a particle (or a particle's graviton) with the same g-factor (muon lepton can be interpreted as inflated electron, or, electron on a different vertical energy level).

Note also that $\sqrt{\left(2^{3}\right)} \mathrm{r}_{s}$ gives a radius of $3411 \mathrm{~km}$, very close to the estimated radius of a discontinuity between outer core and mantle (3486 $\mathrm{km})$.

The charge radius could be understood as the radius of an real graviton whose gravitational potential has been exchanged for electro-magnetic. It is likely, however, that Earth's real charge radius is a superposition of 
multiple charge radii (with opposite charge likely to exist at some inner inner core discontinuity).

Mercury's mass radius has been calculated similarly, with the assumption of $\mathrm{g}_{s}=274 / 2^{2} \mathrm{~m} / \mathrm{s}^{2}=68.5 \mathrm{~m} / \mathrm{s}^{2}$, based on inner core constraints from recent[18] studies[19].

For a Venus' mass radius, the $\mathrm{g}_{s}=274 / 2^{3} \mathrm{~m} / \mathrm{s}^{2}=34.25 \mathrm{~m} / \mathrm{s}^{2}$ has been used, giving $\mathrm{r}_{s}$ in agreement with tidal constraints[20].

For Mars, the $\mathrm{g}_{s}=274 / 2^{4} \mathrm{~m} / \mathrm{s}^{2}=17.125 \mathrm{~m} / \mathrm{s}^{2}$ has been used, this gives a radius roughly $250 \mathrm{~km}$ smaller than current estimates on Mars' core radius $(1810-1860 \mathrm{~km}[21])$.

This result then suggests that the corresponding energy level is likely not the current energy level and a solid inner core at some inner radius may be forming at this time on Mars (assuming current energy level $[\mathrm{n}]$ is lower). In that case, possible radii, ordered by probability (from highest to lowest) are $1118 \mathrm{~km}(\mathrm{n}=3), 790.9 \mathrm{~km}(\mathrm{n}=2), 559.2 \mathrm{~km}(\mathrm{n}=1), \ldots$

Pluto's mass radius was calculated with the assumption of ratio between inner core radius and surface radius equal to Earth's.

Apparently, complexity (excess mass percentage) of terrestrial bodies can be determined by the following equation:

$$
\begin{gathered}
p=\ln \left[n * 10^{36-(2 j+5 i)} e^{(k r)} r^{-1}\right][\%] \\
k=\frac{\pi}{10} \\
i=\sum_{x=0}^{j} x \\
r=d * 10^{-9} \\
\quad \mathrm{~d}=\text { distance of a body from the Sun }[\mathrm{m}]
\end{gathered}
$$

with parameters shown in Table 4. Note that complexity function has a

\begin{tabular}{c|c|c|c|c|r}
$\mathrm{n}$ & $\mathrm{j}$ & $\mathrm{i}$ & body & distance from the Sun $\mathrm{d}\left[10^{9} \mathrm{~m}\right]$ & complexity $\mathrm{p}(\%)$ \\
\hline 1 & 0 & 0 & Mercury & 57.9 & 97.0 \\
3 & 1 & 1 & Venus & 108.2 & 97.2 \\
3 & 2 & 3 & Earth & 149.6 & 82.2 \\
1 & 3 & 6 & Mars & 227.9 & 66.2 \\
3 & 17 & 153 & Pluto & 5906.4 & 91.1
\end{tabular}

Table 4: Complexity parameters for terrestrial bodies 
maximum at Venus or somewhere between Venus and Earth (which is commonly referred to as habitable zone). However, it will have other maxima, depending on relation between $\mathrm{j}$ and $\mathrm{r}$.

Note also that orbital distances of terrestrial bodies scale the same as energy levels of planetary gravitons (with a square root of $2^{n}$ ):

$$
\begin{gathered}
d_{n}=d_{0} * \sqrt{2^{n}} \\
d_{0}=38 * 10^{9} m \text { (terrestrial planets) } \\
d_{0}=8.2 * 10^{9} \mathrm{~m} \text { (terrestrial dwarf planets) }
\end{gathered}
$$

This is not surprising with self-similar universes. It is even possible that gravitons of terrestrial planets initially were inflated to the scale (radius) of current orbital distance, only to collapse (deflate, or localize) afterwards to orbiting spin momenta while coupling with real mass.

Note that $\mathrm{d}_{0}$ should be different for different species of bodies as their graviton wavelengths are of different scale. Chosen $\mathrm{d}_{0}$ for terrestrial dwarf planets is based on $\mathrm{n}=19$ for Pluto. In reality both parameters may be different.

Calculated distances are shown in Table 5. Similar to discontinuities in

\begin{tabular}{l|l|l|l|l}
$\mathrm{n}$ & $\mathrm{j}$ & body & $\begin{array}{l}\text { distance from the } \\
\text { Sun } \mathrm{d}\left[10^{9} \mathrm{~m}\right]\end{array}$ & $\begin{array}{l}\text { calculated distance } \\
\mathrm{d}_{n}\left[10^{9} \mathrm{~m}\right]\end{array}$ \\
\hline 1 & 0 & Mercury & 57.9 & 53.7 \\
3 & 1 & Venus & 108.2 & 107.5 \\
4 & 2 & Earth & 149.6 & 152.0 \\
5 & 3 & Mars & 227.9 & 215.0 \\
19 & 17 & Pluto & 5906.4 & 5937.4
\end{tabular}

Table 5: Orbital distances of terrestrial bodies

planets, it is expectable that current orbital distances will somewhat deviate from radii corresponding to energy levels.

Interestingly, calculated distance for Venus is exactly equal to its perihelion distance, while calculated distance for Earth is almost equal to its aphelion distance $\left(152.1 * 10^{9} \mathrm{~m}\right)$.

This strongly suggests that either perihelia or aphelia of a planet is a fossilized energy level radius (initial orbital distance).

Also interesting is that calculated distances for Mercury and Mars show higher deviation (from both semi-major and perihelia/aphelia) suggesting that 
Mercury and Mars are different species of planets from Venus and Earth (implying somewhat different $\mathrm{d}_{0}$ ), which is not surprising given the order of magnitude different mass.

For these two, with $\mathrm{n}$ unchanged, $\mathrm{d}_{0}$ of $40.6 * 10^{9} \mathrm{~m}$ would give much better results for a semi-major axis - distance of $57.4 * 10^{9} \mathrm{~m}$ for Mercury and $229.7 * 10^{9} \mathrm{~m}$ for Mars. Splitting of energy levels cannot be excluded, thus, better agreement with a semi-major for Mercury and Mars might indicate their perihelia and aphelia correspond to energy levels created with splitting of [semi]major energy levels. Note that orbitals of both have significant eccentricity compared to orbitals of Venus and Earth.

Orbital distances for other bodies can be calculated similarly. In case of outer planets, Uranus and Neptune are likely to belong to same species.

\subsection{Event horizon value (EH operator)}

Inversion (anti-alignment) is common between entangled gravitons. Polarization (inversion) can be interpreted as a result of splitting of a relative event horizon (superposition of gravitons) into gravitons with anti-aligned components of momenta, which thus includes inversion of scale.

Note that inner and outer planets have relatively anti-aligned components of momenta. Inversion is relatively weak between horizontal energy levels, it is stronger between universes (vertical energy levels).

Such splitting (entanglement) may be described by the splitting operator, one of which is the $\mathrm{EH}$ operator:

$$
\begin{aligned}
E H_{N}(a, b) & =\frac{c}{d} \frac{d+1}{c-1} a=\frac{c}{d} \frac{d-1}{c+1} b \\
a & =\frac{d-1}{c+1} \frac{c-1}{d+1} b
\end{aligned}
$$

where $\mathrm{N}=\mathrm{c} / \mathrm{d}$ is the event horizon order and both $\mathrm{c}$ and $\mathrm{d}$ are generally integers.

The inverse value:

$$
\left[E H_{N}(a, b)\right]^{-1}=E H_{N^{-1}}(e, f)
$$

Assuming the inverse must satisfy the following condition:

$$
\frac{E H_{N}(a, b)}{\left[E H_{N}(a, b)\right]^{-1}}=\frac{c}{d} \frac{d+1}{c-1}
$$

this gives:

$$
\left[E H_{N}(a, b)\right]^{-1}=a
$$




$$
\frac{d}{c} \frac{c+1}{d-1} e=\frac{d}{c} \frac{c-1}{d+1} f=a
$$

Since one of the parameters can be omitted, the following notations may be used:

$$
E H_{N}(a, b)=E H_{N}(a)=E H_{N}(, b)
$$

\subsection{Intelligence}

Intelligence is the ability of an individual to focus its consciousness and produce objective (logical) thoughts or conclusions optimally correlated in problem solving.

If nothing can exist without relativity, relativity must exist in intelligence too.

To conserve this relativity, two main classes of intelligence exist: extroverted and introverted.

Due to self-similarity of universes and entanglement between different scales, self-similar reality exists on different scales. For strongly extroverted species everything that happens on smaller scales (relative to body size) is virtual and inaccessible. Thus, extroverted species need external stimulation of senses to perceive reality.

Introverted species do not need external stimulation and may generally be more energy efficient organisms. In extreme cases, introverted organisms are most of time closed self-sustaining systems, do not have limbs, most expressed organ is the brain while other organs are subdued and mainly used to support brain function.

Generally, introverted organisms may be more intelligent, but with subdued external expression may not be considered intelligent or, in extreme cases, even alive at all - by extroverted organisms.

This does not imply that introverted organisms cannot sense the external reality at all (they must be sensitive to radiation at some scale), they just do not act in it with large scale energies.

Generally, life-forms are hybrids (superposition) of extroverted and introverted intelligence. This is evident by the existence of dreams in extroverted species, however, lack of conscious control and consistency make these virtual experiences for most.

However, near-death experiences suggest that introversion generally feels more real than conventionally perceived (extroverted) reality[22].

While complexity of the brain can be correlated with consciousness, apparently, increasing complexity of physical expression beyond the brain is dragging, or blurring, consciousness. 
External expression of intelligence of an individual is always a product of superposition of intelligence of individual cells and proteins.

Each class of intelligence has two components: material and spiritual. Intelligence is polarized when one component is higher than the other.

\subsubsection{Material intelligence}

Material intelligence $\left(\mathrm{I}_{M}\right)$ is the amount of intellectual capacity used to ensure survival of the body of matter (short-term survival).

\subsubsection{Spiritual intelligence}

Spiritual intelligence $\left(\mathrm{I}_{S}\right)$ is the amount of intellectual capacity used to ensure survival of the soul (self-similarity) coupled to the body (long-term survival).

\subsubsection{Intelligence potential (real intelligence)}

The intelligence potential (IP) is a measure of neutral (non-biased) intelligence.

The IP is plastic, and, in polarized (disease prone) individuals, can be strongly affected by diseases (such as depression).

Generally, a function for determination of IP should look something like this:

$$
\begin{array}{r}
I P=\frac{1}{\Delta I} \\
\Delta I=\left|\frac{1}{I_{S}}-\frac{1}{I_{M}}\right| \\
I_{S}+I_{M}=1 \\
\mathrm{I}_{M}=\text { normalized material intelligence } \\
\mathrm{I}_{S}=\text { normalized spiritual intelligence } \\
\mathrm{I}_{S}, \mathrm{I}_{M} \in \mathbb{Q}>0
\end{array}
$$

Note that for $\mathrm{I}_{S}=\mathrm{I}_{M}$ this produces infinity. Since absolute physical infinity is impossible, such result can only be obtained due to limited precision in measurement. Therefore, this infinity should be taken relative and proportional to precision.

\subsubsection{Intelligence quotient (amount of extroverted intelligence)}

Intelligence quotient (IQ) is a conventional measure of externally expressed (extroverted) intelligence. While intelligence potential is invariant to form of intelligence, IQ and similar variants (ie. EQ) are a measure of such intelligence projected to (entangled with) external reality. 
While IQ might correlate well with IP for extroverted species, it is not well suited for extremes and is completely inadequate for measurement of [introverted] intelligence of introverted species.

Some species of animals on Earth may possess higher amount of consciousness and intelligence than humans. It may just not be generally expressed externally.

Signs of complex intelligence are diversity and coherence in brains, or brain equivalents, not in physical expression on generally observable scale.

\subsection{Life}

A form of life is a coupling of a soul (graviton) and a body.

Everything existing is alive but amount of life will be relative to the observer (even uncoupled graviton is always relatively uncoupled, not absolutely), as well as classification of that life.

Coupling of physical and mental components with different ratios of physical to mental activity suggests two main classes - extroverted and introverted life, correlated with extroverted and introverted intelligence.

In general, any life-form is a hybrid of extroverted and introverted life, although generally one form may dominate.

In extremely extroverted life on particular scale brain, or brain equivalent organisation, is extremely subdued (or at least coherence of its components), distinct individual consciousness is minimal and has no influence on physical processes of the body (or the organic collective forming the body).

In life-forms that have developed introversion, brain will dominate and will be able to influence physical processes of the body through mental pathways, affect the constituent organs and collective consciousness of smaller organisms forming its biome.

In extreme extroversion, development of the organism from conception is driven dominantly by interpretation of physical genetic code, such as DNA.

In complete introversion, there is no conventional physical genetic code evolving the individual (generally, however, such code will be involved in development and evolution of individuals forming its biome), instead, development (evolution) of the collective into distinct individuality is driven (or guided) by the interpretation of mental genetic code - the code stored within the soul particle.

Mechanism involved is likely recursive entanglement, starting with entanglement of a soul with a superposition (which is a physical graviton at some scale) of genomes of biome individuals (effect on superposition is reflected in individuals).

However, evolution of either, body or soul, requires coupling of the two. In extreme extroversion, it is the body that will effectively control the soul 
evolution (development), in extreme introversion, vice versa.

Note that all terms are relative, even "mental" and "physical" - mental is physical at some scale, and vice versa.

Planets, in example, are probably extremely introverted organisms - there is no apparent large scale physical DNA equivalent involved in development of a planet even though evolution of its biome is relatively equivalent to DNA coded embryonic development. The equivalence is there because souls and bodies coevolve, influence and mirror each other (albeit with a phase shift).

Nature does not hide anything. Contrary. Things one cannot see on a small scale, are shown on a big screen. But one might need to collapse its ego-system to see all these systems as living eco-systems.

\subsection{God}

Each lifeform whose rest mass (energy) is by one or multiple orders of magnitude greater than life that lives on it and in it, is, for that life, a god.

While the notion of gods is commonly reserved for metaphysical contexts of religion, where the lack of proper definition may be generally desirable, definition of a god correlated with physical phenomena can deepen the understanding of some existing religions and provide meaning to those who are not satisfied with hidden agenda behind materialistic rituals periodically organized to worship untouchable interpretations.

\subsection{Constant}

A constant is a property of a system, non-changeable in particular space and/or time domain (nothing is constant over all space and time). Depending on the size of domain, constant may be weak or strong.

Naturally, all system properties oscillate. Existence of constants is thus relative to observable resolution of space/time by the particular observer.

\subsection{Proper reference frame}

Nothing is absolutely at rest and observation of evolving phenomena will be more common and useful than relatively non-changing phenomena. However, 
relative constants are desirable as reference points and to reduce the complexity of interpretation where influence of that complexity on the outcome is negligible.

A suitable reference is then one relative to which the constancy of variables $\left(\mathrm{x}_{i}\right)$ of the observable system is maximal:

$$
\lim \sum\left(\Delta x_{i}(n)>\hbar_{n}\right)=0
$$

However, due to scale invariance and relativity of constants, proper interpretation will sometimes require a proper reference frame rather than simply a suitable one. Generally to be used in comparison of systems of differently scaled, but otherwise, equal species. In case of polarized frames, a proper [neutral] reference frame may be required even in case of systems of equal scale, to provide more accurate (objective) view of reality.

\section{Quantized inflation of angular momentum}

It was previously hypothesized that radius of a neutral (weakly polarized) graviton scales with a square root of $2^{n}$, where $\mathrm{n}$ is a positive or negative integer. This can be inferred from quantization and scaling of graviton momentum.

Assuming that graviton is inflating from a boson whose momentum projection on a particular axis of quantization (or, when components perpendicular to that axis are annihilated) is $1 \hbar$ while other component (or superposition of other components) is equal in value and perpendicular to that axis, the value of total momentum will be $\sqrt{2} \hbar$ :

$$
L=m v r=\sqrt{2} \hbar
$$

Note that this is equivalent to superposition of two aligned fermions:

$$
L=\sqrt{l(l+1)} \hbar=\sqrt{2 \frac{1}{2}\left(2 \frac{1}{2}+1\right)} \hbar=\sqrt{2} \hbar
$$

However, a $1 \hbar$ momentum can also be a result of superposition of antialigned spin- 2 and spin- 1 bosons.

Since there are no absolutely massless and absolutely neutral particles in CR, coupling of spin-2 gravitons and spin-1 photons should be intrinsic, the difference will only be in scale. Gravity and electro-magnetic force are thus generally coupled and one force may be exchanged for the other but none is absolute 0 in any coupling.

If scale difference is large, ie. in electron the electric force dominates, one component can be considered as relative 0 .

The strong confinement of quarks in protons can be interpreted as intrinsic entanglement strongly evolved between two forces. 
If that momentum is inflated with conserved ratio between values of constituent components of the vector (non-dimensional ratios seem to be generally well preserved during inflation/deflation), proper relativistic treatment here involves either scaling of metric or the $\hbar$ constant in such way that the value of the constant in the current vertical energy level is, in case of inflation, equal in value to momentum in previous energy level. In example, for a single jump in energy level, new momentum is:

$$
L_{n}=\sqrt{2} \hbar_{n}=\sqrt{2} L_{n-1}=\sqrt{2}\left(\sqrt{2} \hbar_{n-1}\right)=\sqrt{2}^{n} \hbar_{0}=\sqrt{2^{n}} \hbar_{0}
$$

Assuming now that speed of an non-coupled graviton is equal to the speed limit of space $\left(c_{n}\right)$ that speed is relatively constant for energy levels of similar magnitude (if, however, the metric is scaled and the ratio between space and time units is conserved, the speed is locally invariant to any scale).

With rest mass remaining constant (relative 0 for non-coupled graviton), the radius must scale the same as the momentum:

$$
r_{n}=\sqrt{2}\left(\sqrt{2} r_{n-1}\right)=\sqrt{2^{n}} r_{0}
$$

Note that inflation of momentum will require additional energy apart from rest energy of inflating quanta. However, sometimes the exchange of momentum components will result in inflation of one component and deflation of the other with no significant difference in the value of momentum. Due to low energy triggers (relative 0) such inflation/deflation (ie. mass oscillation) should generally be inherent for all energy although it may not always be apparent due to time dilation correlated with scale (in non-scaled metric).

As even such inflation must result in some change in momentum (even if generally negligible), this type of scale inversion may also be referred to as inflation/deflation of momentum.

Note that energetic inflation/deflation generally occurs with annihilation of pairs of particles on original scale. The process can be symmetric (producing particle/anti-particle pair with equal mass) or asymmetric (producing particle/anti-particle pair with asymmetric mass distribution).

Inflation/deflation is not limited to bosons or boson pairs. Inflation of a fermion (generally paired with exchange of electro-magnetic potential for gravitational) will similarly scale the original momentum, in which case radius might not scale with $\sqrt{2}^{n}$. During the process, possibility may exist for some components of momentum to annihilate with others preserved (partial annihilation), producing hybrid momenta.

Note that, at full capacity, imaginary mass is equal to real mass, angular velocity is Keplerian and equal to:

$$
v_{n}=\sqrt{\frac{G M}{r_{n}}}=\sqrt{\frac{G}{r_{n}}\left(m_{i m g}+m_{r e}\right)}=\sqrt{2} \sqrt{\frac{G}{r_{n}} m_{i m g}}
$$

Adjacent Keplerian velocities thus also scale with $\sqrt{2}$. If Keplerian velocities are quantized, escape velocity then has to be equal to Keplerian velocity of the next higher energy level of the velocity component. 


$$
v_{e}=v_{n+1}=\sqrt{2} v_{n}=\sqrt{2} \sqrt{\frac{G M}{r_{n}}}=\sqrt{2}\left(\sqrt{2} \sqrt{\frac{G}{r_{n}} m_{i m g}}\right)
$$

Note that, if $\mathrm{r}_{n}$ scales with a square root of $2^{n}$, this velocity corresponds to Keplerian velocity at $\mathrm{r}_{n-2}$.

\section{Discrete states of invariance (energy levels)}

Discrete states of a universe are stable states with relatively invariant (entangled) mechanics. There are vertical and horizontal discrete states (energy levels).

In horizontal states, [energy] difference between levels is generally of the same order of magnitude.

In vertical states, difference is in multiple orders of magnitude.

One example of horizontal states are electron energy levels in atom, whereas two vertical states are an atom and a planetary system.

\subsection{Progression of states}

Progression of discrete states of scale invariance is exponential. For horizontal states, in the top-bottom approach (energy inversely proportional to $\mathrm{n}$ ):

$$
E_{n}=\frac{E_{1}}{n^{2}}=E_{n-1}\left(\frac{n-1}{n}\right)^{2} ; n>1, E_{1}=\text { const. }
$$

For vertical states (energy proportional to n):

$$
\log \left(E_{n}\right)=\log \left(E_{n-1}\right)+(n+1) n=\log \left(10^{(n+1) n} E_{n-1}\right)
$$

For example, mass of Neptune $\left(\mathrm{U}_{1} . \mathrm{e}\right)$ can be obtained from standard electron $\left(\mathrm{U}_{0} . \mathrm{e}\right)$ mass:

$$
\begin{gathered}
\log \left(M_{N}\right)=\log \left(M_{e}\right)+(n+1) n ; n=7, M_{e}=9.10938356 * 10^{-31} \mathrm{~kg} \\
M_{N}=10^{\log \left(M_{e}\right)+8 * 7}=0.910938356 * 10^{26} \mathrm{~kg}
\end{gathered}
$$

Mass of the equivalent particle on $n=6$ level can also be obtained from electron mass:

$$
\begin{gathered}
M_{e}=10^{\log \left(M_{p_{e}}\right)+7 * 6} \\
M_{p_{e}}=10^{\log \left(M_{e}\right)-7 * 6}=9.10938356 * 10^{-73} \mathrm{~kg}
\end{gathered}
$$


This particle is likely to be a component of photons, a half-photon or $\mathrm{U}_{-1}$ electron $\left(\mathrm{U}_{-1} \cdot \mathrm{e}\right)$.

Assuming symmetric pairing of positive and negative charge, mass of the standard photon is then roughly twice this mass:

$$
M_{p}=1.821876712 * 10^{-72} \mathrm{~kg}
$$

I hypothesize that the Solar System is the equivalent of a Carbon-10 $\left({ }^{10} \mathrm{C}\right.$ ) atom. It has been inflated from $\mathrm{U}_{0} \cdot{ }^{10} \mathrm{C}$ state (or even $\mathrm{U}_{-1} \cdot{ }^{10} \mathrm{C}$ ) and electro-magnetic potential has been exchanged for gravitational in the process. Evidence is presented here and in follow up works.

Of course, the values obtained above will deviate slightly from current values due to required oscillation (significant time dilation of oscillation on large scale) and the fact that energy of the outermost electron in Carbon-10 (corresponding to Neptune) slightly differs from the free electron energy used above.

Note that levels calculated above may be considered major vertical energy levels - there are smaller ones. In example, lepton mass oscillation can be considered as oscillation between minor discrete vertical energy levels, or sublevels.

Note also that rest mass is relative - it may be localized to a particle but it may be spread in a wave-form when its mass can be considered as 0 relative to space.

In effect, Neptune is a [vertically] excited electron, and electron is the excited photon [scale] electron.

It is obvious now that the [ 42 orders of magnitude] difference between electric and gravitational force strength between two electrons is sourced in the difference in rest mass between the standard photon [electron] and electron, also 42 $\left(7^{*} 6\right)$ orders of magnitude.

Note that photons can consist of differently scaled particles. But different interpretations are also possible.

If the strength of electric force is invariant to mass of interacting particles, carrier photons produced with proton-electron interaction can be considered as having the same mass as those produced in electron-positron interaction. However, that might not be the case in reality. Gravity carrier particles (gravitons) can couple to half-photons, in which case mass of one half-photon in proton-electron interaction is inflated and photon can be considered as a carrier of gravity too.

Instead of half-photon mass inflation, another possibility is that a 
positron is produced (as intermediate force carrier particle) in protonelectron interaction.

Both interpretations may be equally present in reality.

Coupling to space-forming gravitons obviously enables mass oscillation of particles such as photons.

Note also that entanglement between charges in never absolutely lost. Motion of electrons outside of atoms (or ions in general) will still result in emission of paired half-photons as long as that motion is relative to space of some opposite charge (emission of energy is generally the result of motion relative to space). In case of electro-magnetic energy space is quantized into magnetic field tubes with density of tubes increasing with distance from the source charge (volume of the tubes remains constant in one interpretation of weak entanglement so they are thinning with distance). Distance between the tubes is distance between discrete energy levels and only at infinite distance from the source (assuming unlimited force range) distance between the tubes would be zero. Emission of radiation outside of atoms is thus still the result of energy level changes relative to some other charge.

When localized within the atom on a particular energy level motion of the electron is coupled to angular momentum of specific magnetic field tube(s), electron is at rest relative to space and there is no photon emission. The reason there is no photon emission due to electron motion relative to protons of other atoms must be dragging or distortion of weaker tubes by the local field (note that proton field is effectively dragging the electron field with the electron).

Note also that motion of electron on a particular energy level inside the atom, due to no resistance, is equivalent to motion of charge in superconductors where magnetic field tubes are expelled outwards.

Similar to coupling of electron half-photons (polarized gravitons) to proton half-photons in case of electro-magnetic energy, non-polarized electron gravitons are coupled with non-polarized proton gravitons inside the atom.

However, the scale of gravitons forming space of standard $\left(\mathrm{U}_{0}\right)$ protons and electrons may generally be negligible compared to photons. On $\mathrm{U}_{1}$ scale this is inverted.

Changes in energy level within the $\mathrm{U}_{0}$ atom are likely generally synchronized with temporary inversion of half-photon orbital angular momenta. In $\mathrm{U}_{1}$ this is inversion of graviton orbital angular momenta. Retrograde orbits are then the result of graviton momentum inversion and are unstable if graviton momentum is not aligned with real mass momentum. Even if electro-magnetic and gravitational potential may be generally inverted between adjacent vertical energy levels, localized reversal of inversion is possible on either level.

In one interpretation, it is relative motion that creates (inflates) polariza- 
tion. Boson condensates on $\mathrm{U}_{0}$ scale could then represent such localized reversals with limited range.

With a change in vertical energy level, nature of the force evolves, exchanging polarization (electro-magnetic potential) for neutral gravitational potential or vice versa, depending on the direction of evolution.

Similar to neutral pions, standard photon is composed of a particle/antiparticle pair (ie. $\mathrm{U}_{-1} \cdot \mathrm{e}^{-} / \mathrm{U}_{-1} \cdot \mathrm{e}^{+}$). These have anti-aligned $1 / 2$ spin momenta, with aligned orbital momenta forming total spin of the photon.

Note the following:

$$
M_{p_{e}}=\frac{M_{e}}{M_{N}} K_{A}=\frac{M_{e}}{M_{N}} 1.02413 * 10^{-16} \mathrm{~kg}
$$

where $\mathrm{M}_{N}=1.02413 * 10^{26} \mathrm{~kg}$ is the mass of Neptune, and quantum of mass $\mathrm{K}_{A}=1.02413 * 10^{-16} \mathrm{~kg}\left(5.7 * 10^{19} \mathrm{eV}=57 \mathrm{EeV}\right)$ is the mass (energy) of asymmetry.

If standard electron mass would be equal to current value of $\mathrm{K}_{A}$ and halfphoton would have mass equal to $1.02413 * 10^{-58} \mathrm{~kg}$, the adjacent electron energy levels to $\mathrm{U}_{0}$ would be symmetric relative to the electron.

Symmetry could also be achieved simply by decreasing half-photon mass to $8.1 * 10^{-87} \mathrm{~kg}$ ( setting $\mathrm{K}_{A}=\mathrm{M}_{e}=9.10938356 * 10^{-31} \mathrm{~kg}$ ).

If vertical asymmetry needs to be preserved, the asymmetry breaking energy of $\mathrm{K}_{A}-\mathrm{M}_{e} \approx \mathrm{K}_{A}=5.7 * 10^{19} \mathrm{eV}$ must be the energy limit for standard particles produced in any universe conforming to the above progression of vertical states as that energy could, through annihilation, inflate electron mass to $\mathrm{K}_{A}$.

Note that symmetry by decrease of half-photon mass requires considerably more energy $-8.9 * 10^{-15} \mathrm{~kg}\left(4.99 * 10^{21} \mathrm{eV}=4988 \mathrm{EeV}\right)$.

At first, it might seem unclear why would it be necessary to preserve vertical asymmetry, but the reason is equivalent to the reason for preservation of horizontal asymmetry.

Particles with mutually inverted properties annihilate when distance between them is reduced to relative 0 . Whether this annihilation occurs within the 
atom or outside the atom, it has to occur at particular energy level. This thus requires symmetry relative to that level. Since adjacent vertical energy levels have inverted properties, symmetry could lead to annihilation of adjacent energy levels.

Different scales (universes) are entangled (correlated) and if this entanglement is physical (in CR, it has to be) than that entanglement can be interpreted as a dimension in space.

If correlated but anti-aligned properties are universally attracted and relatively annihilated when at equal distance from a particular energy level, conservation of existence requires conservation of asymmetry in scale.

Note that energy levels are physical. In case of electro-magnetic energy, energy levels are magnetic field tubes. Splitting of energy levels can then be interpreted as splitting of magnetic field tubes, although alternative interpretations are possible.

Creation of symmetry would require adjustment of half-photon mass in either case, therefore the energy of $5.7 * 10^{19} \mathrm{eV}$ may be interpreted as the 1 st order limit, with the 2 nd order of $4.99 * 10^{21} \mathrm{eV}$.

Studies indeed confirm the 1st order limit as the cut-off energy[23] for intragalactic sources[24].

It is also in agreement with measurements of GZK (Greisen-Zatsepin-Kuzmin) energy limit (cut-off) for protons - 5.6 $\pm 0.5 \pm 0.9 * 10^{19} \mathrm{eV}[25]$.

Interestingly, particles with higher energy have been detected, on the order of $10^{20} \mathrm{eV}$, but no particle has been produced or observed exceeding the $2 \mathrm{nd}$ order limit.

Effectively, thus, the relative superposition of the 1st and 2nd order may be interpreted as real limit.

In any case, increasing number of particles with such high energies could indicate instability of the observable universe.

As mass depends on the number and mass of coupled gravitons rest mass is different at different energy levels. This is not limited to vertical energy levels, although in case of horizontal levels difference may generally be negligible.

Changes in energy levels are generally correlated with changes in frequency. Consider photons. Even though rest masses of constituent halfphotons are quantized, their orbital momenta must be proportional to frequency if speed of photons is invariant to frequency.

Rest masses of gravitons are quantized too and will be different on different energy levels, but probable rest masses can be deduced assuming scale-invariance of non-dimensional mass ratios. These ratios should be well preserved between 
vertical energy levels.

In example, one likely rest mass of an $\mathrm{U}_{-1}$ scale graviton can be deduced from the ratio of an $\mathrm{U}_{0}$ graviton rest mass and electron mass (as noted before, at full capacity, mass of the graviton is half the total mass):

$$
\begin{aligned}
M_{n}=\frac{\frac{1}{2}^{10} C \text { atom mass }}{\frac{1}{2} \text { electron mass }} M_{p}=\frac{{ }^{10} C \text { atom mass }}{\text { electron mass }} M_{p} \approx \frac{10.016853 u}{M_{e}} M_{p} & =2 * 1.663337576 * 10^{-68} \mathrm{~kg} \\
& =3.326675152 * 10^{-68} \mathrm{~kg}
\end{aligned}
$$

Obviously, half of mass of an atom [nucleus] is not the mass of a single graviton, rather a superposition of multiple gravitons, however, such superposition on $\mathrm{U}_{-1}$ scale is likely to be interpreted as elementary from $\mathrm{U}_{0}$ reference frames.

Different atoms with different masses can be interpreted as having gravitons with different masses, however, masses of these gravitons may be limited to a couple of generations and difference in atomic mass could be the difference in real mass (over-capacity, under-capacity).

Another interpretation, however, may be more likely.

If gravitons are oscillating in rest mass between different vertical sublevels, oscillation may be such that a superposition of oscillating mass produces the net gravitational effect matching coupled real mass.

In more massive nuclei, gravitons would simply spend more time inflated to higher energy levels.

Complexity (diversity of charge coupling) in strong force should be correlated with this oscillation as it is coupled with exchange between electromagnetic and gravitational potential.

Note that the obtained mass is in agreement with other calculations of graviton mass[26] $\left(\approx 10^{-68} \mathrm{~kg}\right)$, based on existing theories and observation.

Assuming that $\mathrm{U}_{0}$ gravitational mass has been inflated from $\mathrm{U}_{-1}$ scale, dark matter of $\mathrm{U}_{0}$ scale can be represented by the inflated superposition of $\mathrm{U}_{-1}$ gravitons (not coupled with real mass).

In that case, assuming the superposition is interpreted as a single graviton, it's mass is at least roughly equal to half the ${ }^{10} \mathrm{C}$ atom mass. More likely, however, is inflation of pairs of such gravitons, giving mass roughly equal to ${ }^{10} \mathrm{C}$ mass:

$$
U_{0} \cdot M_{n}=10.016853 u=1.663337576 * 10^{-26} \mathrm{~kg}
$$

The mass corresponds to energy of $9.33 * 10^{9} \mathrm{eV}(9.33 \mathrm{GeV})$ and evidence exists in favour of such dark matter particles[27]. 
Note that there is no single dark matter particle. Any naked graviton of any scale is a particle of dark matter.

Note also that [rest] masses of standard photons and neutrinos may generally be determined from momentum, relative to c constant.

Masses obtained here are invariant to c. Obtained half-photon mass corresponds to a particle of vacuum energy density $\left(9.9 * 10^{-27} \mathrm{~kg} / \mathrm{m}^{3}\right)$, with a radius of $\mathrm{U}_{0}$ scale Neptune equivalent $\left(\approx 3.8343 * 10^{-16} \mathrm{~m}\right)$ and orbital velocity of $\approx 3.5 * 10^{26} \mathrm{~m} / \mathrm{s}$.

Physically, a particle with such momentum can be obtained with a collapse of a gravitational maximum of $\mathrm{U}_{0}$ Neptune equivalent (standard electron) to $\mathrm{U}_{-1}$ scale energy, converting mass to velocity to conserve momentum (note that this speed would be valid even in GR, if photon [scale electron] is understood as quantum of vacuum - here it does have vacuum energy density).

Rest photon mass relative to standard c (when its spin momentum is not taken into account) can then be obtained through conservation of momentum $\left(\mathrm{p}=\mathrm{m}_{1} \mathrm{v}=\mathrm{m}_{2} \mathrm{c}\right)$, and it is $\approx 2.1 * 10^{-54} \mathrm{~kg}$.

This mass agrees with experimentally obtained photon mass through its interaction with matter[28].

But the obtained photon rest mass of $1.821876712 * 10^{-72} \mathrm{~kg}$ can also be validated through conservation of energy. In CR, relativistic speed limits are different between different scales of energy.

For standard protons and electrons speed limit is the standard speed of light (c), however, for photons with a rest mass obtained above, speed limit must be much higher. The reason we don't directly observe photons travelling at greater speeds may be one of the following:

- faster photons do not directly interact with atoms (quantum entanglement, however, could be interpreted as indirect observation of superluminal photons although this could also be interpreted as stretching of [units of] space),

- their linear or angular momentum is effectively limited by density/pressure of [static gravitons forming] space.

In reality, both interpretations are possible, however, spin momentum of photons should not be limited by c and will be proportional to photon frequency (gravitons forming photon's space have much smaller radii and are orbiting at speeds exceeding c). Thus, any momentum that would exceed the limit imposed by space is converted to photon's spin momentum.

In any case, photon with obtained rest mass travelling at speed close to $\mathrm{c}$ is non-relativistic (uncoupled photons with speeds much lower than $\mathrm{c}$ would be, due to pressure of static gravitons, relativistic - speed c could be interpreted as their rest velocity). Assuming then, a photon is travelling parallel to Earth's surface in Earth's atmosphere [reference frame], 
at the moment of its coupling with a gas molecule in air, conservation of energy will yield:

$$
\begin{gathered}
m_{1} v_{1}^{2}=m_{2} v_{2}^{2} \\
m_{1} c^{2}=m_{2} v_{2}^{2} \\
m_{2}=\frac{m_{1} c^{2}}{v_{2}^{2}}
\end{gathered}
$$

$\mathrm{m}_{1}=$ photon mass before coupling $=1.821876712 * 10^{-72} \mathrm{~kg}$ $\mathrm{v}_{1}=$ photon velocity before coupling $=\mathrm{c}=2.99792458 * 10^{8} \mathrm{~m} / \mathrm{s}$

$\mathrm{v}_{2}=$ photon velocity after coupling $\mathrm{m}_{2}=$ photon mass after coupling

Thermal velocity of air is $464 \mathrm{~m} / \mathrm{s}$ and velocity of a molecule the photon is interacting with will generally have similar velocity. Assuming velocity of the molecule increases to $464.3 \mathrm{~m} / \mathrm{s}$ with coupling to the photon, velocity $\mathrm{v}_{2}$ is $464.3-464=0.3 \mathrm{~m} / \mathrm{s}$.

This yields:

$$
m_{2}=1.82 * 10^{-54} \mathrm{~kg}
$$

Of course, this is valid for a photon of specific rest mass (frequency), in general, conservation of total momentum (spin and orbital/linear) should be used (or correlation of energy with frequency), however, coupling [rest] masses should have discrete values - excess spin momenta will then be transformed to kinetic/thermal energy.

Further validation of calculated masses and velocities can be found in the analysis of the Solar System in CR context[8] (chapter Quantum nature: Outermost angular momenta and $c_{1}$ confirmation) and other follow-up papers[29].

\subsubsection{Oscillation of photon mass}

Mass of all particles should be oscillating and photon cannot be an exception. Its constituent particles (half-photons) should, as leptons, oscillate between 3 generations.

Assuming calculated mass is the lowest mass, other two can be calculated from tau/muon/electron mass ratios (these should be well preserved between vertical energy levels).

For $\mathrm{M}_{\tau}=1776.86 \mathrm{MeV} / \mathrm{c}^{2}, \mathrm{M}_{\mu}=105.6583755 \mathrm{MeV} / \mathrm{c}^{2}$ and $\mathrm{M}_{e}=0.511$ $\mathrm{MeV} / \mathrm{c}^{2}$ :

$$
M_{\gamma \tau}=\frac{M_{\tau}}{M_{e}} M_{\gamma e}=\frac{1}{2} 6.335068208 * 10^{-69} \mathrm{~kg}
$$




$$
\begin{array}{r}
M_{\gamma \mu}=\frac{M_{\mu}}{M_{e}} M_{\gamma e}=\frac{1}{2} 3.767055455 * 10^{-70} \mathrm{~kg} \\
M_{\gamma e}=\frac{M_{p}}{2}=\frac{1}{2} 1.821876712 * 10^{-72} \mathrm{~kg} \\
M_{\gamma e}=\text { electron half-photon rest mass } \\
M_{\gamma \mu}=\text { muon half-photon rest mass } \\
M_{\gamma \tau}=\text { tau half-photon rest mass }
\end{array}
$$

To prevent annihilation, masses of two constituent particles should be equal for 0 time relative to other combinations (relative 0 ). The oscillation of one halfphoton should thus be anti-aligned with the other (phase difference of $180^{\circ}$ in ideal case) and the whole system (photon) can be reduced to two-body oscillation. Here the intermediate ( 0 time) state must be the state with lowest mass (state lifetime is proportional to mass).

Note that, there must exist a threshold frequency - at which point two half-photons will fuse and form a graviton half-particle.

Due to non-zero mass, photon must have a range, roughly equal to [reduced] Compton wavelength, ie.:

$$
\begin{array}{r}
r=\frac{\hbar}{M_{p} c}=\frac{\hbar}{c} \frac{1}{M_{\gamma \tau}+M_{\gamma \mu}} \\
\begin{array}{r}
\hbar=\mathrm{h} /(2 \pi)=1.054573 * 10^{-34} \mathrm{Js} \\
\mathrm{c}=2.99792458 * 10^{8} \mathrm{~m} / \mathrm{s}
\end{array}
\end{array}
$$

Massive particles will generally either gain or lose energy in motion.

There are various interpretations for loss of energy of a particle such as photon:

- due to required oscillation of velocity, at times the speed limit imposed by space may be broken - resulting in radiation of energy of smaller scale,

- small scale temperature - energy of smaller scale will be radiated away if the environment is of lower temperature,

- perceived density of space cannot be absolutely zero (particles may be coupling with gravitons),

- transfer of momenta (ie. through gravitational interaction) to other small scale particles in space. 
If motion is orbital, energy will generally be cyclically replenished. However, in reference frames where that motion is linear, loss or gain of energy will be observed.

Obviously, $\mathrm{r}$ must also be roughly equal to the radius of observable universe (universe that can be observed) - when calculated for lowest rest photon mass at rest frequency.

Assuming mass oscillation is synchronized with coupling of photons to gravitons, both photon velocity and frequency will change with gravitational potential.

Acceleration of emitted photons must also oscillate but may be effectively constant with high frequency of mass oscillation. For effective mass equal to $\mathrm{M}_{\gamma \tau}+\mathrm{M}_{\gamma \mu}$, acceleration is:

$$
a= \pm \frac{1}{2} c^{2} \frac{c}{\hbar}\left(M_{\gamma \tau}+M_{\gamma \mu}\right)= \pm \frac{1}{2} \frac{c^{3}}{\hbar}\left(M_{\gamma \tau}+M_{\gamma \mu}\right)= \pm 4.287091748 * 10^{-10} \frac{\mathrm{m}}{\mathrm{s}^{2}}
$$

For $2 \mathrm{M}_{\gamma e}$ :

$$
a= \pm \frac{1}{2} \frac{c^{3}}{\hbar} 2 M_{\gamma e}= \pm \frac{c^{3}}{\hbar} M_{\gamma e}= \pm 2.327418326 * 10^{-13} \frac{\mathrm{m}}{\mathrm{s}^{2}}
$$

Generally, taking into account phase shift and using relativistic (gravitational) mass, the equation becomes:

$$
\begin{aligned}
& a= \pm \frac{1}{2} \frac{c^{3}}{\hbar}\left(\frac{1}{2}+\frac{1}{2} \sin ^{2} \phi\right) \Delta M \frac{1}{\sqrt{1-\frac{f^{2}}{f_{n}{ }^{2}}}} \\
& \Delta M^{2}=2^{2} \frac{M_{1}^{2}+M_{2}^{2}}{2}=2\left(M_{1}^{2}+M_{2}^{2}\right)
\end{aligned}
$$

where $\Delta \mathrm{M}$ is the superposition of mass, $\mathrm{f}$ is photon spin frequency, $\mathrm{f}_{n}$ is the maximum possible photon spin frequency and $\varphi$ is a mixing angle (equal to $90^{\circ}$ for aligned and $0^{\circ}$ for anti-aligned oscillation of half-photons). For previously determined 1st order maximum energy of $\mathrm{M}_{n}=1.02413 * 10^{-16} \mathrm{~kg}\left(5.7 * 10^{19}\right.$ $\mathrm{eV}), \mathrm{f}_{n}$ is:

$$
f_{n}=\frac{c}{\lambda_{n}}=\frac{E_{n}}{h}=\frac{M_{n} c^{2}}{h}=1.389120683 * 10^{34} \mathrm{~Hz}
$$

Calculated acceleration for various combinations and $\mathrm{f} \ll \mathrm{f}_{n}$ is shown in Table 6. Acceleration of photons may be misinterpreted as a change in Doppler shift (change in velocity of the source of emission) when mass of the photon is considered to be 0 . 


\begin{tabular}{l|l|l|l}
$\mathrm{M}_{1}$ & $\mathrm{M}_{2}$ & $\varphi\left[^{\circ}\right]$ & $\mathrm{a}\left[\mathrm{m} / \mathrm{s}^{2}\right]$ \\
\hline $\mathrm{M}_{\gamma \mu}$ & $\mathrm{M}_{\gamma \mu}$ & 90 & $\pm 0.481235302 * 10^{-10}$ \\
$\mathrm{M}_{\gamma \mu}$ & $\mathrm{M}_{\gamma \mu}$ & 0 & $\pm 0.240617651^{*} 10^{-10}$ \\
$\mathrm{M}_{\gamma \tau}$ & $\mathrm{M}_{\gamma \mu}$ & 90 & $\pm 5.732686887 * 10^{-10}$ \\
$\mathrm{M}_{\gamma \tau}$ & $\mathrm{M}_{\gamma \mu}$ & 0 & $\pm 2.866343444 * 10^{-10}$ \\
$\mathrm{M}_{\gamma \tau}$ & $\mathrm{M}_{\gamma \tau}$ & 90 & $\pm 8.092948194 * 10^{-10}$ \\
$\mathrm{M}_{\gamma \tau}$ & $\mathrm{M}_{\gamma \tau}$ & 0 & $\pm 4.046474097 * 10^{-10}$
\end{tabular}

Table 6: Acceleration for various photon mass eigenstates

There are two interpretations of photon acceleration (deceleration). In one interpretation photon linear momentum remains effectively constant - the change in energy (frequency) is reflected in momenta of its constituent quanta. However, changes in spin momenta are generally correlated with changes in orbital/linear momenta and acceleration of photons will be a superposition of both interpretations, only the dominant component will vary, depending on properties of space.

Indeed, analysis of motion of Pioneer 10/11, Galileo, and Ulysses spacecraft shows anomalous relatively constant weak long-range acceleration of $(8 \pm 3) * 10^{-10} \mathrm{~m} / \mathrm{s}^{2}$ (deceleration relative to the Sun) for which no satisfactory explanation has been found[30], with the assumption that photon mass is 0 .

Obviously, predicted photon mass oscillation due to gravitational potential can fully explain this anomaly.

However, if the anomaly is the result of photons coupling to gravitons (dragging by dark matter), masses - and therefore accelerations, in calculations above should be doubled or paired, depending on interpretation.

There are two interpretations.

Either photon mass can be considered as relative 0 while graviton is considered as superposition of two of above calculated masses, or the photon mass is real mass while gravitons inflate to match that mass.

Recently, dark matter was indeed proposed as the solution to the problem[31].

However, in that solution no effect on photons is considered, rather on spacecraft exclusively.

It should be questionable, however, whether these gravitons form the 
gravitational potential of the Solar System, or the galaxy (as proposed, effectively, by the cited work).

Anomalies have been detected on other spacecrafts. Table 7 shows the anomalies and calculated accelerations for matching graviton configurations. Evidently, the predicted mass oscillation is in remarkable agreement with ob-

\begin{tabular}{|c|c|c|c|c|}
\hline spacecraft & $\begin{array}{l}\text { distance } \\
{[\mathrm{AU}]}\end{array}$ & anomaly $\left[\mathrm{m} / \mathrm{s}^{2}\right]$ & $\begin{array}{l}\text { possible graviton con- } \\
\text { figurations }\left[\mathrm{M}_{1}, \mathrm{M}_{2},\right. \\
\varphi]\end{array}$ & $\begin{array}{l}\text { calculated acceler- } \\
\text { ation }\left[\mathrm{m} / \mathrm{s}^{2}\right]\end{array}$ \\
\hline $\begin{array}{l}\text { Pioneer } \\
(<1999)\end{array}$ & $40-60$ & $8.09 \pm 0.20 * 10^{-10}$ & $2^{*}\left[\mathrm{M}_{\gamma \tau}, \mathrm{M}_{\gamma \tau}, 0^{\circ}\right]$ & $\begin{array}{l}8.092948194 \\
10^{-10}\end{array}$ \\
\hline $\begin{array}{l}\text { Pioneer } \\
(<1999)\end{array}$ & $<30$ & $8.56 \pm 0.15 * 10^{-10}$ & $\begin{array}{l}{\left[\mathrm{M}_{\gamma \tau}, \mathrm{M}_{\gamma \tau}, \quad 90^{\circ}\right]+} \\
{\left[\mathrm{M}_{\gamma \mu}, \mathrm{M}_{\gamma \mu}, 90^{\circ}\right]}\end{array}$ & $\begin{array}{l}8.574183496 \quad * \\
10^{-10}\end{array}$ \\
\hline $\begin{array}{l}\text { Ulysses } \\
1995)\end{array}$ & $1.3-5.2$ & $12 \pm 3 * 10^{-10}$ & $\begin{array}{l}{\left[\mathrm{M}_{\gamma \tau}, \mathrm{M}_{\gamma \tau}, 90^{\circ}\right]+} \\
{\left[\mathrm{M}_{\gamma \tau}, \mathrm{M}_{\gamma \mu}, 0^{\circ}\right]} \\
{\left[\mathrm{M}_{\gamma \tau}, \mathrm{M}_{\gamma \tau}, 90^{\circ}\right]+} \\
{\left[\mathrm{M}_{\gamma \tau}, \mathrm{M}_{\gamma \mu}, 90^{\circ}\right]}\end{array}$ & $\begin{array}{l}12.139422291 * 10^{-10} \\
13.825635081 * 10^{-10}\end{array}$ \\
\hline Galileo (1993) & 3 & $8 \pm 3 * 10^{-10}$ & $2 *\left[\mathrm{M}_{\gamma \tau}, \mathrm{M}_{\gamma \tau}, 0^{\circ}\right]$ & $\begin{array}{l}8.092948194 \quad * \\
10^{-10}\end{array}$ \\
\hline Pioneer 10 (2003) & 82 & $8.74 \pm 1.33 * 10^{-10}$ & $\begin{array}{c}2^{*}\left[\mathrm{M}_{\gamma \tau}, \mathrm{M}_{\gamma \tau}, 0^{\circ}\right] \\
{\left[\mathrm{M}_{\gamma \tau}, \mathrm{M}_{\gamma \tau}, 90^{\circ}\right]+} \\
{\left[\mathrm{M}_{\gamma \mu}, \mathrm{M}_{\gamma \mu}, 90^{\circ}\right]}\end{array}$ & $\begin{array}{l}8.092948194 * 10^{-10} \\
8.574183496 * 10^{-10}\end{array}$ \\
\hline $\begin{array}{l}\text { New } \\
(2008)\end{array}$ & 8 & $13.2 \pm 0.6 * 10^{-10}$ & 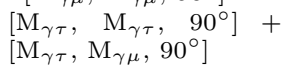 & $\begin{array}{l}13.825635081 \\
10^{-10}\end{array}$ \\
\hline
\end{tabular}

Table 7: Anomalies and proposed solutions (anomaly data sources: $\mathrm{a}^{30}, \mathrm{~b}^{31}$ )

servations.

Interestingly, the results suggest that in all solutions masses should be equally aligned (both either $0^{\circ}$, or $90^{\circ}$ ). This would then imply that Ulysses anomaly is the same in value to New Horizons anomaly.

Generally, any particle with finite range (mass $>0$ ) should be converting radial component of velocity to angular orbital velocity with distance relative to the source unless the range is continuously extended by mass loss.

This is a consequence of entanglement (and physical interpretation of that entanglement on particular scale!) of emitted particles with the source, however, entanglements can change (decrease/increase).

\subsubsection{Additional evidence for photon mass and its oscillation}

Observed acceleration is a solid evidence for photon rest mass in predicted range $\left(10^{-68}-10^{-72} \mathrm{~kg}\right)$.

However, generally, photon mass may be obtained from linear (angular) momentum. Since most of its effective mass is in spin momentum $\left(\mathrm{v} \approx 10^{26}\right.$ $\mathrm{m} / \mathrm{s}$ ), experimentally obtained photon mass will be significantly higher than the 
calculated rest mass. Using conservation of momentum, one can calculate these masses for various pairs of half-photons, ie:

$$
\begin{gathered}
m_{1} v=m_{2} c \\
M_{\tau \tau}=6.335068208 * 10^{-69} * 3.5 * 10^{26} * \frac{1}{c}=7.39603 * 10^{-51} \mathrm{~kg} \\
M_{\mu \mu}=3.767055455 * 10^{-70} * 3.5 * 10^{26} * \frac{1}{c}=4.39794 * 10^{-52} \mathrm{~kg} \\
M_{e e}=1.821876712 * 10^{-72} * 3.5 * 10^{26} * \frac{1}{c}=2.12699 * 10^{-54} \mathrm{~kg} \\
\mathrm{c}=2.99792458 * 10^{8} \mathrm{~m} / \mathrm{s}
\end{gathered}
$$

Indeed, experimentally obtained photon masses range from $10^{-50}$ to $10^{-54}$ $\operatorname{kg}[28]$.

Note that here the same velocity has been used for all photons. In reality, some difference in orbital velocity of half-photons may exist, especially for heavier half-photons where it could result in inflation of photon mass to the order of $10^{-50} \mathrm{~kg}$ or beyond.

\subsubsection{Photon/graviton range}

Obviously, photon can indeed travel vast distances and that would not be possible if its rest mass would range from $10^{-50}$ to $10^{-54} \mathrm{~kg}$.

However, as gravitons exist on different scales, this range of masses could represent another vertical sublevel. If so, these would have the following spatial ranges:

$$
\begin{gathered}
r_{\tau \tau}=\frac{\hbar}{c} \frac{1}{M_{\tau \tau}}=47561.69 \mathrm{~km} \\
r_{\mu \mu}=\frac{\hbar}{c} \frac{1}{M_{\mu \mu}}=7.99846493 * 10^{8} \mathrm{~m} \\
r_{e e}=\frac{\hbar}{c} \frac{1}{M_{e e}}=1.653828596 * 10^{11} \mathrm{~m}
\end{gathered}
$$

These ranges are interesting because they could be correlated with celestial bodies. Ie. for a photon/graviton mass of $5.52 * 10^{-50} \mathrm{~kg}$ the range would be equal to Earth's radius. 
I hypothesize that such particles do exist and are carriers of electro-magnetic and gravitational force for large scale $\left(\mathrm{U}_{1}\right)$ gravitons.

Photons with lower rest masses $\left(10^{-68}-10^{-72} \mathrm{~kg}\right)$ are carriers of such force for real mass $\left(\mathrm{U}_{0}\right.$ scale) - atoms, filling the wells of large scale maxima.

In that case, empty large scale gravitational wells should be devoid of, not only standard atoms, but these photons/gravitons too (they would be transparent for such photons but would be unable to produce them due to absence of real mass) unless escape velocity of a maximum is $>c$ (black hole maximum).

This implies that gravity of such wells has a very limited range.

However, there are other interpretations - perhaps the very shielding effect of real mass in a well manifests itself in the reduction of range (rest mass increase) of constituent gravitons of the well once these are absorbed (coupled).

Indeed, that would be the most plausible explanation for shielding effect, assuming it exists.

The strong nuclear force then becomes a result of exchange of large source rest mass ( $10^{11} \mathrm{~kg}$ black hole) for large force carrier mass, resulting in range decrease (collapse).

Proton radius can then be interpreted as a fossilized black hole radius. This has to be one of the most fascinating features of the observable universe - not showing any care for absolute numbers, it's shuffling energies regardless of their magnitudes, all while leaving fossils behind as memories left for someone to process sometime in space and somewhere in time.

\subsection{Lorentz factor}

In $\mathrm{CR}$, relativistic corrections must be relative themselves. Scale invariance (vertical energy levels) implies change of metric [units]. Without this change, one must change the value of constants accordingly. Thus, the Lorentz factor cannot be valid for all scales.

In GR, metric may be scaled proportionally to energy, but scaling is limited to space and time dimensions (mass itself doesn't scale in GR, leaving other constants absolute). Another problem is that the constant of proportionality between space and time is interpreted as an absolute constant - invariant to scale. In CR, this cannot be a correct interpretation.

In conventional (absolute) form, the Lorentz factor allows a body of infinite mass to move, bound by the same speed limit as any other body of mass. 
Since such mass requires infinite energy just to start moving, its speed limit is, however, effectively 0 .

Similarly, a particle of absolute zero mass should have a speed limit equal to infinity as it has absolute 0 mass relative to space.

Of course, one could interpret change in metric of GR as change in energy density - for a non-local observer, infinite mass would indeed not move in GR due to infinite time dilation, while a massless particle in infinitely flat space would have infinite velocity. However, one cannot assume that there exists only one and absolute space-time entanglement (at least not in CR).

One can use such reference frame in calculations, but this will lead to misinterpretation of reality on large and small scales.

According to CR, space and time both must be quantized (force carrier particles have quantized and non-zero rest masses). This, effectively, makes curvature of space-time relative to scale.

Space is thus relatively continuous (due to infinite number of carrier particles) but also relatively quantized (due to quantized ranges and entanglements of carriers with different sources).

The infinite number of space-time entanglements only reduces to GR interpretation if the ratio between space and time scales is absolutely equal to $\mathrm{c}$ in all the entanglements.

This, generally, won't be true, even if it is a good approximation for close scales.

Speed limit may be determined from density and pressure of space. Since density and pressure of space are relative [to scale], for a body of infinite mass, density and pressure are such that speed limit is 0 .

If standard photons are quanta of space they will (or can) travel at the speed limit (c) determined from pressure and density of such space only if they don't have mass relative to that space.

However, if they do have kinetic energy relative to that space they must have mass, therefore must travel at speed $<$ c. Effectively, while in motion relative to space, they are no longer quanta of space.

Note that this implies that a photon travels at rest velocity as quantum of space, while its own space can spin at higher velocity (»c, as calculated) unless that velocity is exchanged for radius (which should be the case if photon travels as a wave).

The photons in CR, however, cannot be created, they can be inflated. Most likely, half-photon, as a polarized component of space, has an orbital angular velocity equal to c, while it's spin velocity is much higher. Only at time of photon creation, when half-photon is paired with another half-photon, the total radius is inflated at speed c. At time of photon absorption (localization), radius collapses, but with the inflation of mass instead of increasing spin due to present velocity relative to space. In example, coupling of a photon with an atom [graviton] the velocity of 
the photon relative to space becomes equal to that of the atom.

This explains why all standard photons and neutrinos as waves travel at equal speed - their superluminal spin velocity has been exchanged for radius while radius inflation is equally limited. But is it limited by $\mathrm{c}$ ? In $\mathrm{CR}$, c should be interpreted as average velocity of inflation. If rest masses of neutrinos and photons are different, the final radius (range) of inflation will be different and so may be the average velocity unless both photon and neutrino have the same source and are detected [collapsing radius] at the same location.

On small enough distances, average velocity should be higher than c, on long enough distances lower than c.

All static standard photons thus have a spin momentum with angular velocity »c. This makes the magnetic spin momentum of electron intuitive - its polarized space is indeed rotating at speeds $>c$ and this space has a finite radius.

Note that half-photon, as a quantum of polarized space, is a magnetic field tube.

Speed limits for particular mass are thus relative to [the scale of] that mass and speed limits can relatively be broken.

The relative breaking of a speed limit is synchronized with a change in rest mass energy level, when speed is adjusted to match rest velocity on that level, while speed limit changes to the value associated with the new level.

The Lorentz factor should thus have the form:

$$
\gamma=\frac{1}{\sqrt{1-\frac{v^{2}}{c_{n}{ }^{2}}}}
$$

when applied to rest mass:

$$
\begin{gathered}
\gamma_{m}=\frac{m_{n}}{\sqrt{1-\frac{v^{2}}{c_{n}^{2}}}} \\
\text { generally, } m_{n} \propto \frac{1}{c_{n}}
\end{gathered}
$$

where $\mathrm{n}$ is the vertical energy level.

Increasing mass [dimension] is thus increasing time dimension while decreasing (quantizing) space dimension (or less likely, keeping one constant while changing other). This can then be interpreted as exchange between space and time which can be correlated with exchange between gravitational potential and electro-magnetic potential or exchange between gravitational mass (range) and carrier mass. Space-time is then an entanglement of quanta or quantum of space orbiting a quantum or quanta 
of time. Each can be localized at their rest orbital, while in between they may generally travel non-localized (inflating/deflating radii).

Change in speed limit thus requires emission or absorption of a minimum discrete amount (impulse) of energy which can take the body to another vertical energy level.

This allows for planetary systems such as the Solar System to be inflated atoms or a system of bound, but individually inflated particles.

Note that inflation must be sufficiently fast to preserve the structure, but it cannot be infinitely fast to break the inevitable asymmetry.

It is likely that such inflation is triggered by matter/anti-matter annihilation (similar to annihilation/inflation of standard photons into standard electron/positron pairs).

Such event is both, the moment of death, and birth, since it likely occurs in the space of a dark matter particle pair (soul, gravitational maximum) where one pair is inflated and the other collapses (exchange of souls) - ie. a collision occurs at $U_{0}$ energy level, one pair inflates to $U_{1}$, other collapses to $U_{-1}$.

Since there are no absolute universes, any universe must be inflating in another universe, thus any anomalous energies might have a source in that larger universe.

The speed limit of $\mathrm{c}=2.99792458 * 10^{8}$ is the speed limit for $\mathrm{U}_{0}$ particles (ie. standard electrons) in space of $U_{1}$ bodies, speed limit for $U_{1}$ scale is generally lower, while for $U_{-1}$ scale particles (standard photons) in space of $U_{0}$ bodies it is higher (note that this implies that charge of the electron is rotating in space formed by $\mathrm{U}_{-2}$ scaled quanta).

Standard photons can travel faster than c even though one may not be able to detect such photons directly. However, spin magnetic momentum of electron should be interpreted as evidence of half-photons (photon scale electrons) orbiting at velocities faster than c.

This is a proper interpretation in CR and it is a proper interpretation if one wants to conserve rationality (intuition).

\subsubsection{Determination of $c_{n}$}

Once the scales of space and time are known, one can easily obtain $c_{n}$.

It was hypothesized previously that $\mathrm{U}_{1}$ scale is entangled with $\mathrm{U}_{-1}$ scale ( $\mathrm{U}_{-1}$ gravitons form the space of $\mathrm{U}_{1}$ gravitons).

Generally, space of an $\mathrm{U}_{n}$ graviton is formed by $\mathrm{U}_{n-2}$ gravitons, thus:

$$
c_{n}=c_{n-1}\left(\sqrt{\log \left(m_{n}\right)^{2}}+\sqrt{\log \left(m_{n-2}\right)^{2}}\right)^{-1}
$$

The speed limit $\mathrm{c}_{n}$ on scale $\mathrm{U}_{1}\left(\mathrm{U}_{1} \cdot \mathrm{c}\right.$ or $\left.\mathrm{c}_{1}\right)$ is then:

$$
c_{1}=c_{0}\left(\sqrt{\log \left(m_{1}\right)^{2}}+\sqrt{\log \left(m_{-1}\right)^{2}}\right)^{-1}=c_{0}\left[\log \left(M_{N}\right)-\log \left(M_{p_{e}}\right)\right]^{-1}
$$




$$
\begin{array}{r}
\mathrm{c}_{0}=\mathrm{c}=2.99792458 * 10^{8} \mathrm{~m} / \mathrm{s} \\
\mathrm{M}_{N}=1.02413 * 10^{26} \mathrm{~kg} \\
\mathrm{M}_{p_{e}}=9.10938356^{*} 10^{-73} \mathrm{~kg}
\end{array}
$$

which, for $c_{0}$ equal to standard speed of light (c), $\mathrm{m}_{1}$ equal to Neptune's mass and $\mathrm{m}_{-1}$ equal to calculated half-photon mass gives:

$$
c_{1}=2.99888155 * 10^{6} \mathrm{~m} / \mathrm{s}
$$

Of course, within any vertical energy levels there are sublevels (mass oscillation). In example, for $\mathrm{U}_{1} .3$ sublevel the speed limit is:

$$
c_{1.3}=c_{0}\left[\log \left(M_{N}\right)+10^{\frac{m-1}{m}}-\log \left(M_{p_{e}}\right)\right]^{-1}=2.91932312 * 10^{6} \mathrm{~m} / \mathrm{s}
$$

$\mathrm{m}=3$

Taking into account horizontal oscillation, ie. $\mathrm{U}_{1 .} .3$ level, with the assumption of a quantum of energy $\mathrm{U}_{1} .3 .3$ lost:

$$
\begin{array}{r}
c_{1.3}=c_{0}\left[\log \left(M_{N}\right)+10^{\frac{m-1}{m}}-\left(\frac{k-1}{k}\right)^{2}-\log \left(M_{p_{e}}\right)\right]^{-1}=2.93201263 * 10^{6} \mathrm{~m} / \mathrm{s} \\
\mathrm{m}=3, \mathrm{k}=3
\end{array}
$$

Generally, it would probably be useful to limit c to the average value (reference frame) for a particular level, in this case, the value of $2.93 * 10^{6} \mathrm{~m} / \mathrm{s}$ may be a valid candidate for average on $\mathrm{U}_{1}$ level.

If one is comparing equivalent systems between scales, a precise value can be calculated assuming strongly non-dimensional ratios are well preserved (ie. space/space, not space/time) and knowing precise values of some variables in space and time between two systems, or between one system and the intermediate equivalent.

The limit on scale $\mathrm{U}_{-1}$ can be calculated similarly, assuming entanglement of adjacent scales:

$$
\begin{gathered}
c_{-1}=c_{0}\left(\sqrt{\log \left(m_{0}\right)^{2}}+\sqrt{\log \left(m_{-2}\right)^{2}}\right) \\
m_{-2}=10^{\log \left(M_{p_{e}}\right)-6 * 5}=9.10938356 * 10^{-103} \mathrm{~kg} \\
\mathrm{~m}_{0}=\mathrm{M}_{e}=9.10938356 * 10^{-31} \mathrm{~kg}
\end{gathered}
$$




$$
c_{-1}=3.96 * 10^{10} \mathrm{~m} / \mathrm{s}
$$

Speed limit on $\mathrm{U}_{-2}$ :

$$
\begin{gathered}
c_{-2}=c_{-1}\left(\sqrt{\log \left(m_{-1}\right)^{2}}+\sqrt{\log \left(m_{-3}\right)^{2}}\right) \\
m_{-3}=10^{\log \left(m_{-2}\right)-5 * 4}=9.10938356 * 10^{-123} \mathrm{~kg} \\
c_{-2}=7.69 * 10^{12} \mathrm{~m} / \mathrm{s}
\end{gathered}
$$

This limit is interesting as $U_{-2}$ particles should form the charge of standard electron $\left(\mathrm{U}_{0} \cdot \mathrm{e}\right)$. The magnetic moment of the electron cannot be formed by rotation of charge exceeding this velocity (without deflating to smaller scale).

The magnetic spin angular velocity of electron can be calculated assuming charge radius is known. Assuming this radius is a scaled Neptune charge radius and assuming the Solar System is the carbon atom equivalent (a hypothesis explored in greater detail in follow-up work), the radius is:

$$
\begin{gathered}
R_{e}=R_{0}=\frac{R_{1}}{r_{1}} * r_{0}=3.834298096 * 10^{-16} \mathrm{~m} \\
\mathrm{R}_{1}=\text { Neptune charge radius }=24622000 \mathrm{~m} \\
\mathrm{r}_{1}=\text { Neptune orbital radius }=4495.06 * 10^{9} \mathrm{~m} \\
\mathrm{r}_{0}=\text { orbital radius of the outermost electron in carbon atom }=70^{*} 10^{-12} \mathrm{~m}
\end{gathered}
$$

Angular velocity of charge forming the quantized momentum of electron is then:

$$
v=\frac{1}{2} \frac{1}{M_{e} R_{e}} \hbar=1.5096 * 10^{11} \mathrm{~m} / \mathrm{s}
$$

The charge is thus rotating at allowed velocity (not exceeding $\mathrm{c}_{-2}$ ).

Note that electron at standard scale $\left(\mathrm{U}_{0}\right)$ may not generally orbit the atom as a particle rather as a wave. In that case, due to conservation of momentum, the velocity of its charge is much lower as the radius $\mathrm{R}_{e}$ becomes equal to atom radius $\left(\mathrm{r}_{0}\right)$.

Its charge, however, may not be distributed as an orbital wave-line, rather orbital wave-surface, which would then increase velocity somewhat (by $3 / 2$ maximum) but this is negligible compared to initial decrease. Generally, its charge forms a spinning tube.

Closer to nucleus the charge velocity may become relativistic but it still won't exceed $c_{-2}$ and probably won't even exceed $c_{0}$ (between the innermost electron and atom centre speeds can be greater than $\mathrm{c}_{0}$ ).

Note how using the standard speed of light $\left(\mathrm{c}=\mathrm{c}_{0}\right)$ would produce a picture inconsistent with reality. If electron would in a wave-form orbit 
the atom at speed $\mathrm{c}_{0}$ the atom radius would have to be $2.9 * 10^{-13} \mathrm{~m}$, some 241 times smaller than it is in reality.

\section{The unification}

In CR, nothing can be absolute, even continuum of space must be, like all energy, a sum of discrete energies or energy levels. Diversity of energy levels implies it has variable and scale relative properties, such as density and pressure. The number of dimensions of space is in foundations of every theory in physics, here, obviously, such number is relatively infinite. Infinite number of scales (dimensions) might exist, however, in CR, intrinsic limitations on observation of scales of energy exist, effectively, making any infinity relative.

Dimensions evolve and one may recognize different species of dimensions where individuals of species often can be represented by the average or relative superposition of individuals.

On small scales even different species might be represented by some kind of superposition.

Generally, it is convenient to define three or four main species - space, mass, time and charge. These are all entangled, but amounts of entanglement will vary and a difference in superposition of the four may itself warrant classification into different species of superposition.

We might use units with different names for the 4 species $(\mathrm{m} / \mathrm{kg} / \mathrm{s} / \mathrm{C})$ but, at some fundamental level, these are generally all units of [angular momenta of] space at some scale and a universe will readily transform one energy into another (exchange space for time, time for mass, etc.).

Here thus, there is no intrinsic entanglement between space-time curvature and mass or charge, the space-time-mass entanglement in GR is thus just one possible case of such entanglement.

\subsection{Relativistic time}

Everything in nature has an angular momentum relative to something. It is natural then that dimensions of nature are circular and cyclic, quantized into different periods and with different shapes.

Even though these dimensions are cyclic there can be no absolute space, time or mass travel - only relative.

In example, dimension of time (or a quantum of dimension of time) may be considered as circular thin tube (or even a relative line), a torus, with relatively 
discrete or relatively continuous degrees of polarization. Space (or a quantum of dimension of space) may be considered as a thin sphere surface, a torus with significant asymmetry in tube width and height - a relative superposition of time dimensions of various radii. Charge - a superposition (localization) of time dimensions. Mass - a superposition (localization) of spaces.

CR implies a relatively simultaneous existence of energy on various vertical scales (self-similarity).

If energy existing on one scale can be aware of, or be sensitive to, adjacent discrete vertical energy levels only, direct sensitivity is strongly limited to 3 dimensions. This will be, with implied self-similarity, replicated on all these levels - splitting these into 3 components, hence explaining three generations of particles.

Note that not all universes have to be limited to direct awareness of 3 scales (splitting of energy can result in more than 3 levels) and even those limited to 3 may be aware of other scales indirectly - which might become direct awareness over time.

Note also that self-similarity can be interpreted as relatively simultaneous existence of energy on various scales.

If nature does not discriminate between scales of energy (just as it does not between units), then entanglement and operations between adjacent scales should also be natural. In which case, the scaling vector may be normalized.

Let $\mathrm{A}$ represent a state in time and $\mathrm{C}$ a scaling vector, the operation of addition between scales (components of time) could then be represented by the scalar product of vectors:

$$
A \cdot C=\left[\begin{array}{llll}
a_{1} & a_{2} & \cdots & a_{n}
\end{array}\right] \cdot\left[\begin{array}{c}
c_{1} \\
c_{2} \\
\vdots \\
c_{n}
\end{array}\right]=a_{1} \cdot c_{1}+a_{2} \cdot c_{2}+\cdots+a_{n} \cdot c_{n}
$$

With normalization, the scaling vector $\mathrm{C}$ becomes a unit vector:

$$
A \cdot \frac{C}{|C|}=\left[\begin{array}{llll}
a_{1} & a_{2} & \cdots & a_{n}
\end{array}\right] \cdot\left[\begin{array}{c}
c_{1} /\left|c_{1}\right| \\
c_{2} /\left|c_{2}\right| \\
\vdots \\
c_{n} /\left|c_{n}\right|
\end{array}\right]=a_{1} \hat{c_{1}}+a_{2} \hat{c_{2}}+\cdots+a_{n} \hat{c_{n}}
$$

Note that, if dimensions here represent scales, geometrical representation of the state with orthogonal unit vectors would be misleading. 
and, with no discrimination between scales:

$$
\hat{c_{1}}=\hat{c_{2}}=\cdots=\hat{c_{n}}=\hat{c}
$$

scales collapse to one dimension:

$$
\vec{A}=\left(a_{1}+a_{2}+\cdots+a_{n}\right) \hat{c}
$$

With no direction, a point in such time becomes a scalar:

$$
A=\left(a_{1}+a_{2}+\cdots+a_{n}\right) c
$$

The collapses of scales in time will have physical interpretations in space and correlations between these may be interpreted as events of synchronicity, however, generally, it should not be assumed that c (speed of change) is absolutely the same between different scales of energy.

\subsection{Relativistic space-time}

In General Relativity space is treated as non-physical, it has a geometry but no variable physical properties such as density and pressure in common interpretations. Space/time entanglement is treated as absolute which translates to an absolute speed limit, equal to vacuum speed of light in case of massless photons:

$$
\begin{gathered}
c=\sqrt{\frac{1}{\epsilon_{0} \mu_{0}}} \\
\epsilon_{0}=\text { vacuum electric permittivity }=8.85418782 * 10^{-12} \mathrm{~F} / \mathrm{m} \\
\mu_{0}=\text { vacuum magnetic permeability }=4 \pi^{*} 10^{-7} \mathrm{H} / \mathrm{m}
\end{gathered}
$$

However, this can be rewritten in terms of energy density $\rho$ and pressure p:

$$
\begin{aligned}
& \epsilon_{0}=K_{\epsilon} \frac{F}{m}=K_{\epsilon} \frac{s^{4} A^{2}}{m^{3} k g}=K_{\epsilon} \frac{s^{4} N^{2}}{m^{3} m^{2} k g T^{2}}=K_{\epsilon} \frac{s^{4} k g^{2} m^{2}}{m^{3} m^{2} k g s^{4}} \frac{1}{T^{2}} \\
& =K_{\epsilon} \frac{k g}{m^{3}} \frac{1}{T^{2}}=K_{\epsilon} \frac{k g}{m^{3}} \frac{1}{T} \frac{C}{N} \frac{m}{s}=\rho_{s} \frac{1}{B_{1}} \frac{1}{E_{1}} v_{1} \\
& \mu_{0}=K_{\mu} \frac{H}{m}=K_{\mu} \frac{m k g}{s^{2} A^{2}}=K_{\mu} \frac{m k g m^{2} T^{2} s^{4}}{s^{2} k g^{2} m^{2}} \\
& =K_{\mu} \frac{m s^{2}}{k g} T^{2}=K_{\mu} \frac{m s^{2}}{k g} T \frac{N}{C} \frac{s}{m}=\frac{1}{p_{s}} B_{2} E_{2} \frac{1}{v_{2}} \\
& \text { for } E_{1} B_{1}=E_{2} B_{2} \text { : } \\
& c=\sqrt{\frac{p_{s}}{\rho_{s}} \frac{v_{2}}{v_{1}}}=\sqrt{\frac{p_{s}}{\omega \rho_{s}}} \\
& \begin{array}{r}
\mathrm{K}_{\epsilon}=8.85418782 * 10^{-12} \\
\mathrm{~K}_{\mu}=4 \pi * 10^{-7}
\end{array}
\end{aligned}
$$


and now in terms of energy E and mass m:

$$
\begin{gathered}
\epsilon_{0}=\rho_{s} v_{1}=\frac{m}{V} v_{1} \\
\frac{1}{\mu_{0}}=p_{s} v_{2}=\frac{E}{V} v_{2} \\
c=\sqrt{\frac{E / V}{m / V} \frac{v_{2}}{v_{1}}}=\sqrt{\frac{E}{m} \frac{v_{2}}{v_{1}}}
\end{gathered}
$$

From this follows:

$$
\begin{gathered}
E=\frac{v_{1}}{v_{2}} m c^{2}=\omega m v_{r}{ }^{2} \\
\omega=\frac{v_{1}}{v_{2}}=\frac{v_{r}}{\sqrt{v_{r}^{2}-v^{2}}}=\frac{1}{\sqrt{1-\frac{v^{2}}{v_{r}^{2}}}}
\end{gathered}
$$

Here, factor $\omega$ is the non-dimensional relativistic factor.

In GR, energy is always relative to an absolute rest frame $\left(\mathrm{v}_{r}=\mathrm{c}\right)$, which may be referred to as vacuum frame or even CMB (Constant Microwave Background) rest frame due to omnipresence of $\mathrm{CMB}$ and negligible photon mass.

The space/time ratio of that frame is considered intrinsic and non-changeable and, assuming $\mathrm{v}_{r}=\mathrm{c}$, with an absolute 0 momentum ( $\mathrm{v}=0$ above).

In QM discrete energy is limited to horizontal levels and bound by counterintuitive limitations of the same absolute rest frame.

In CR there are no such restrictions, no rest frame is absolute, each gravitational well has its own space and there are, not only horizontal but vertical energy levels corresponding to scale of discrete packets of energy.

In $\mathrm{CR}$, the CMB rest frame is space of a large-scale graviton with [angular] velocity $(\mathrm{v})$ possibly equal to $\mathrm{c}$, relative to a rest frame $\mathrm{v}_{r}>\mathrm{c}$, and with a rest mass $<\mathrm{mc}^{2} / \mathrm{v}_{r}{ }^{2}$.

Space and time of this rest frame may be entangled but information between them cannot travel instantaneously. Also, either may change spin and entangle with another dimension.

Even in weak entanglement, where speed of information transfer increases with distance (due to decrease of particle/wave radii), the speed only becomes infinite at infinite distance between entangled dimensions. Decrease in scale in entanglement is increasing probability for establishment of a bigger entanglement with a closer dimension (switch of context). At infinite distance, entanglement is stable for absolute 0 time.

Therefore, the ratio $\mathrm{c}$ is not absolutely intrinsic and it is changeable. 


\subsection{Omega factor (relativistic change)}

Omega factor is a non-dimensional relativistic factor, a generalization of the Lorentz factor. It is a necessary modification in order to allow complete relativity of universes.

Energy is relative to a specific reference frame and omega factor will generally be relative to a specific graviton in whose space energy is contained.

Space of a graviton is characterized by its $\epsilon \mu$ product (or density and pressure).

Omega factor represents change in energy due to momentum:

$$
\begin{gathered}
\omega=\frac{1}{\sqrt{1-\frac{v^{2}}{c_{n}^{2}}}}=\frac{1}{\sqrt{1-\frac{v^{2}}{k^{2} c^{2}}}} \\
\mathrm{c}=\mathrm{c}_{0}=\text { standard speed of light }
\end{gathered}
$$

where $\mathrm{k}$ depends on the vertical energy level (scale of energy).

Note that $\omega^{-1}$ is the eccentricity of the ellipse of width equal to $2 \mathrm{kc}$ and height equal to $2 \mathrm{v}$, as shown in Fig. 5 .

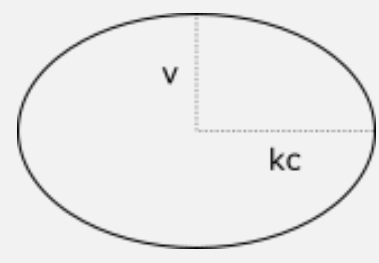

Figure 5: Relativistic ellipse

With $\mathrm{k}=1$, width is fixed to $\mathrm{c}$ and omega factor degenerates to Lorentz. Note also, if $\mathrm{k}$ itself has the form of $\omega^{-1}$, degeneration to Lorentz becomes degeneration of a variable ellipsoid to an ellipse of fixed width.

In GR, for an relativistic ellipse it is absolutely forbidden to form a circle $(\mathrm{v}=\mathrm{kc})$.

However, in CR, this must be only relatively forbidden.

Assume $\mathrm{v}$ is the angular velocity of a rest frame 1 contained within (or relative to) a rest frame 2 which has angular velocity equal to kc.

It is not forbidden for a rest frame 1 to reach velocity kc and no infinite energy for that is needed either (infinity also must be relative) - however, needed energy is equal to energy of rest frame 2 (minus rest frame 1 energy). Thus, rest frame 1 can reach velocity kc by using all available energy of rest frame 2 . This is then simply a transformation or exchange of rest frames - rest frame 1 was born (inflated) into rest frame 2, while rest frame 2 died (deflated) into rest frame 1.

With rest frame 1 becoming rest frame 2 , its velocity is now rest velocity and speed limit now may be increased to angular velocity of rest frame 
3 it is entangled with. Note that entanglement may not be broken. In that case the speed limit now represents a speed minimum and velocity is relativistic if the rest frame slows down.

Since entanglements cannot be absolute 0 , velocity becomes relativistic when it is either increased or decreased from rest velocity. In that case, rest velocity can be interpreted as a velocity where entanglements cancel. This is all relative to some remote reference frame. From the perspective of rest frame 1 , due to scale change, speed limit may be relatively invariant.

Note that energy can also be applied externally, rest frame 2 (generally a graviton) can merge with another such particle, changing its angular velocity and speed limit for rest frame 1.

However, this should be further generalized, to allow polarization of space and summation of (sensitivity to, awareness of) different scales of energy.

In a physical reality in which every universe (or distinct form of energy) has a momentum the energy of which is stored into its gravitational maximum, it is appropriate to introduce the concept of rest velocity for rest frames (or rest spaces), equal to:

$$
\begin{aligned}
v_{r n}= & \sqrt{\frac{1}{\omega \epsilon \mu}} \\
& =\text { rel. const. (ie. } 8.854^{*} 10^{-12} \mathrm{~F} / \mathrm{m} \text { ) } \\
& \mu=\text { rel. const. (ie. } 4 \pi^{*} 10^{-7} \mathrm{H} / \mathrm{m} \text { ) }
\end{aligned}
$$

where

$$
\begin{gathered}
\omega=\omega_{n}=\omega\left(n, q_{0}\right)=\left(\frac{1}{\sqrt{1-\alpha_{n}}}\right)^{-\operatorname{sgn}\left(q_{0}\right)}, \\
\alpha=\alpha_{n}=\sum_{m=1}^{m=\infty} \frac{v^{2}}{v_{r(n-m)^{2}}} \approx \frac{v^{2}}{v_{r(n-1)}{ }^{2}},
\end{gathered}
$$

$\mathrm{n} \in \mathbb{Z}$

$\mathrm{m} \in \mathbb{N}$

and $\mathrm{n}$ is a discrete (vertical) energy level of the rest frame, $\mathrm{v}$ and $\mathrm{q}_{0}$ its velocity and charge (radial polarization), respectively, relative to rest frame chosen by the observer (since awareness of the observer is generally limited to adjacent vertical levels of energy, this frame will usually be $n-1$, and $n-2$ for larger scales of energy).

\subsubsection{Degeneration to Lorentz factor}

For a negatively polarized rest frame, and $\mathrm{U}_{n-2} \ll \mathrm{U}_{n-1}\left(\mathrm{v}_{r_{(n-2)}} » \mathrm{v}_{r_{(n-1)}}\right)$ :

$$
\alpha_{n}=\frac{v^{2}}{v_{r(n-1)^{2}}}
$$


Fixing all discrete packets of energy to single scale where $\mathrm{v}_{r_{n-1}}=\mathrm{v}_{r}=\mathrm{c}$ is the speed limit (speed of light) on that scale, Omega factor becomes Lorentz factor:

$$
\gamma=\frac{1}{\sqrt{1-\frac{v^{2}}{c^{2}}}}
$$

\subsubsection{Effect on charge and mass}

Charge and mass may be commonly exchanged. Generally, charge may be proportional to real mass of the total mass of coupling so this can be interpreted as exchange between real mass and img mass if this is local exchange.

Effective electric charge $q$ and mass $m$ now become:

$$
\begin{gathered}
q=q_{n}=\frac{q_{0}}{\omega} \\
M_{i m g}=M_{n}=\omega M_{0}=\frac{q_{0}}{q} M_{0} \\
M_{r e}=M_{n-1}=\frac{M_{0-1}}{\omega}=\frac{q}{q_{0}} M_{0-1} \\
\left.\mathrm{q}_{0}=\text { total rest charge }=\text { rel. const. (ie. } 1.60217733^{*} 10^{-19} \mathrm{C}\right) \\
\mathrm{M}_{0}=\text { total img rest mass }=\text { rel. const. } \\
\mathrm{M}_{0-1}=\text { total real rest mass }=\text { rel. const. } \\
M=\sum_{i=0}^{\infty} M_{n-i}=\sum_{i=0}^{\infty} \omega^{k} M_{0-i} \approx M_{i m g}+M_{r e} \\
k=k(i)=-1^{(i \bmod 2)}
\end{gathered}
$$

Note 1: All indexed parameters $\left(\mathrm{q}_{0}, \mathrm{M}_{0}, \mathrm{M}_{n}\right.$, etc.) are simply factors of proportion, relative to scale of choice. Since rest state of energy is relative (nothing is absolutely at rest), using recursion, one concludes that the sole intrinsic property of a universe is change (evolution), but in order for it to exist, multiple universes (bigger and smaller scale) are required.

Note 2: Total mass may be decreasing or increasing with relativistic energy, thus, this is generally not a simple local mass exchange. It includes a factor of transformation $(\omega)$ which is exchanging scales of real and img dimensions but with emission of energy (inversely proportional to complexity of transformation) and absorption of additional 
energy - ie. absorption of $\mathrm{m}_{n-2}$ (not previously considered as part of total mass $\mathrm{M}$ ), into $\mathrm{M}_{n}$.

Relativistic effects are always real at some scale, it is only a question to what degree is the effect local or remote. If the observable moves at rest velocity locally, relativistic effect is generally low (small scale) locally.

\subsubsection{Effect on radius}

Effective radius of a gravitational maximum also depends on $\omega$ :

$$
R=\omega R_{0}
$$

$$
\mathrm{R}_{0}=\text { rest radius }
$$

In case of negative polarization it increases with velocity, for positive it decreases.

\subsection{Energy}

A distinct form of energy can be composed of one or more discrete scales of energy. Total energy is:

$$
E=\sum E_{n}=\omega m c^{2}
$$

where $\mathrm{mc}^{2}$ is rest energy relative to a rest frame with rest velocity c, while $\omega$ represents relativistic increase or decrease due to kinetic energy relative to the observer.

Since kinetic energy of a system is stored in gravitons (when relativistic relative to space), its distribution will depend on the scale of these gravitons.

\subsection{General force}

Electro-magnetic and gravitational force are generally entangled and potential should be regularly exchanged between the two. Therefore, it should be useful to couple the two mathematically into a 4 -component vector force, ie. general force.

General force acting on a particle (ie. moon maximum) of mass $\mathrm{m}$, charge $\mathrm{q}$ and velocity $\mathrm{v}$ is the sum of polarized (electro-magnetic) and neutral (gravitational) force.

At any moment in space/time the force acting on a body is a superposition of gravitational and electro-magnetic force. In extreme conditions (temperature/pressure) one component may collapse (deflate) as other inflates. 
Source of the force is a general rotating graviton with its field spread through the well:

$$
\begin{gathered}
\vec{F}=q m \vec{S}+q m \vec{v} \times \vec{B}+q m \vec{E}+q m \vec{G}=q m \vec{S}+q m \vec{M}+q m \vec{E}+q m \vec{G} \\
\vec{F}=q m(\vec{S}+\vec{M}+\vec{E}+\vec{G})
\end{gathered}
$$

with charge/mass radii reduced to infinitesimal value:

Even though the particle has multiple associated charges and real charge/mass radii, to simplify equations, it is useful to reduce it to a point particle, especially in cases where it effectively is a point particle - ie. when space/time resolution is such that real radii or the oscillation in charge/mass distribution cannot be determined.

$$
\begin{aligned}
& \vec{M}=\vec{v} \times \vec{B} \\
& \vec{B}=\frac{1}{m} \mu \frac{q_{r}}{r^{2}} \overrightarrow{v_{r}} \times \frac{\vec{r}}{r} \\
& \vec{E}=\frac{1}{m} \frac{1}{\epsilon} \frac{q_{r}}{r^{2}} \frac{\vec{r}}{r} \\
& \vec{G}=\frac{1}{q} \frac{1}{g} \frac{m_{r}}{r^{2}} \frac{\vec{r}}{r}=\frac{1}{q} \frac{1}{r} v_{s}{ }^{2} \frac{\vec{r}}{r} \\
& \vec{S}=\vec{v} \times\left(\frac{1}{q} s \frac{1}{r} \overrightarrow{v_{s}} \times \frac{\vec{r}}{r}\right) \\
& \epsilon=\text { electric permittivity of space }(* 4 \pi) \\
& \mu=\text { magnetic permeability of space }(/ 4 \pi) \\
& \mathrm{g}=1 / \mathrm{G}=\text { energy density of gravity at the maximum (inverse of the } \\
& \text { gravitational constant) } \\
& \mathrm{s}=\text { relativistic factor } \\
& \mathrm{M}=\text { spin electric field at } \mathrm{r} \\
& \mathrm{B}=\text { magnetic field at } \mathrm{r} \\
& \mathrm{E}=\text { electric field at } \mathrm{r} \\
& \mathrm{G}=\text { gravitational field at } \mathrm{r} \\
& \mathrm{S}=\text { spin gravitational field at } \mathrm{r} \\
& \mathrm{v}_{s}=\text { angular velocity of space (effective graviton) at } \mathrm{r}
\end{aligned}
$$

where $\mathrm{m}_{r}, \mathrm{q}_{r}$ and $\mathrm{v}_{r}$ are mass, charge and velocity, respectively, of the field maximum, $r$ is the distance between the graviton and the moon particle. 
Note that $\mathrm{s}$ is non-dimensional (invariant to scale) and must be equal to:

$$
s=\frac{v_{s} v}{c_{n}^{2}}
$$

For $\mathrm{n}=0\left(\mathrm{c}_{0}=\mathrm{c}\right)$, using substitution:

$$
v_{s}^{2}=\frac{G m_{r}}{r}
$$

, with the angle $\phi$ between $\mathrm{v}$ and $\left(\mathrm{v}_{s} \mathrm{x} \mathrm{r}\right)$ being equal to the angle between $\mathrm{v}_{s}$ and $\mathrm{r}$, the $\mathrm{qmS}$ term reduces to:

$$
q m \vec{S}=\frac{G m_{r} m v^{2}}{c^{2} r^{2}} \sin ^{2} \phi
$$

which, when simplified to one-body problem - using reduced (effective inertial) mass $\left(\mathrm{m}=\mu, \mathrm{m}_{r}=\mathrm{M}+\mathrm{m}\right)$, becomes the correction factor to gravitational potential from General Relativity:

$$
V(r)=\int q m \vec{S} d r=-\frac{G(M+m) \mu v^{2}}{c^{2} r} \sin ^{2} \phi
$$

At extreme momentum change, polarized and neutral components can exchange potential - E exchanges with $\mathrm{G}$, while M exchanges with S.

Note that in equilibrium (full capacity) $\mathrm{v}_{s}=\mathrm{v}\left(\mathrm{v}_{s} \mathrm{v}\right.$ becomes $\left.\mathrm{v}_{s}{ }^{2}\right)$, and, using the above substitution for $\mathrm{v}_{s}{ }^{2}$, spin gravitational vector $\mathrm{S}$ becomes:

$$
\begin{aligned}
\vec{S}=\vec{v} \times\left(\frac{1}{q} \frac{G}{c_{n}^{2}} \frac{m_{r}}{r^{2}} \overrightarrow{v_{s}} \times \frac{\vec{r}}{r}\right) & =\vec{v} \times\left(\frac{1}{q} k \frac{m_{r}}{r^{2}} \overrightarrow{v_{s}} \times \frac{\vec{r}}{r}\right) \\
\mathrm{k} & =\text { specific vacuum density }[\mathrm{m} / \mathrm{kg}]
\end{aligned}
$$

making vector $\mathrm{S}$ the gravitational equivalent of spin electric vector $\mathrm{M}$. Note also that even the ratio between constants of the polarized and neutral force vectors is equal:

$$
\frac{1}{\epsilon} \frac{1}{\mu}=\frac{1}{g} \frac{1}{k}=G \frac{c_{n}{ }^{2}}{G}=c_{n}{ }^{2}
$$


Note that if momenta are quantized on one vertical energy level, they must be quantized on all levels - from a proper (scale invariant) reference frame.

Due to [relatively] low energy oscillations in vertical scale (ie. neutrino, lepton oscillation) caused by the splitting of a vertical level, inflation of a system of multiple bodies may inflate different bodies [of the same species] to different [relatively] low energy levels and quantization might not appear conserved (as timescales might be inadequate to detect oscillation).

Also note that, with a change in level (ie. oscillation), due to finite speed of propagation of changes in space, distant bodies might not feel the same force as local bodies.

\subsubsection{Exchange of quantized potential}

In the chapter above (General force), it was assumed that force carrier particles are massless, which may be a good approximation generally, however, such interpretation won't always paint an accurate picture of reality.

As there are no absolutely massless particles in CR, both gravity and electromagnetic potentials should be Yukawa type potentials. However, as each carrier particle is a source of force it has its own field with appropriate carrier particle of smaller scale. Even though each field is quantized, recursion leads to infinite ranges and zero mass particles.

Thus, each component of general force should be coupled with a sum of Yukawa type terms:

$$
\exp \left(\frac{-r}{L_{n}}\right)
$$

where $\mathrm{L}_{n}$ is the range of a carrier particle (reduced Compton wavelength).

Note that horizontal energy levels may not be treated equally to vertical energy [sub]levels. In example, carrier rest mass may be treated unchangeable across the horizontal energy levels (levels of the same energy magnitude), it may only be considered [relatively] temporarily excited (or, having excited range).

In any case, at stable energy levels, Yukawa term disappears for coupled gravitons.

However, sensitivity (probability for coupling) of real mass to these gravitons will be inversely proportional to difference in scale of energy between the two. 
To take this into account, each Yukawa term should be multiplied by the sensitivity factor, proportional to:

$$
\begin{gathered}
\left(\frac{E}{E_{n}}\right) \delta_{i j}+\left(\frac{E_{n}}{E}\right)\left(1-\delta_{i j}\right) \\
i=\left\lfloor\frac{E}{E_{n}}\right\rfloor, j=0
\end{gathered}
$$

$$
\delta_{i j}=\text { Kronecker delta }
$$

Of course, one should also take into account mass oscillation here.

Therefore, due to quantized ranges, energy levels are quantized, however, due to different scales, quantization is relative. This is evident in planetary systems planetary orbitals are obviously quantized while quantization for smaller bodies is not as obvious due to greater density of energy levels.

Furthermore, the amount of polarization of force carrier particles (gravitons) is likely to change with scale (which may be the source of difference in sensitivity). In that case, distant bodies might effectively feel one force while close bodies would feel other force. Nuclear atomic force, when interpreted as general force, is one such example - on close range gravity dominates, otherwise, electro-magnetic force.

Exchange of one potential for the other has two interpretations - either charge of the field source is exchanged with mass, or energy is exchanged between polarized and non-polarized space (mass/charge exchange in carrier particles).

In reality, superposition of both, should be present.

\subsubsection{Solution to gravitational anomalies}

Previously, it was assumed that angular velocity of space (effective graviton) $\mathrm{v}_{s}$ at radius $r$ is equal to Keplerian velocity:

$$
v_{s}^{2}=\frac{G M}{r}
$$

And that velocity is also assumed to be the velocity of standard matter coupled to that graviton. This assumption is equal to the assumption that mass of the effective graviton is always equal to the mass of coupled standard matter. However, this should be valid only at full capacity states - when all constituent quanta of the effective graviton (gravitons of smaller scale) are coupled to matter.

Suppose the equation of state is:

$$
G M \frac{m_{s}}{v_{s}}=m v r
$$




$$
\begin{array}{r}
\mathrm{G}=\text { gravitational constant } \\
\mathrm{M}=\text { mass enclosed within radius } \mathrm{r}
\end{array}
$$

where the right side is orbital angular momentum of matter, while $\mathrm{m}_{s}$ and $\mathrm{v}_{s}$ are mass and velocity of the effective graviton, respectively.

The effective graviton is forming the toroidal quantum of space which the matter is traversing, its mass $\mathrm{m}_{s}$ is thus the total mass of constituent gravitons while $\mathrm{v}_{s}$ is their average velocity.

From (G1.1), orbital velocity of matter is:

$$
v=G M \frac{m_{s}}{m} \frac{1}{v_{s} r}
$$

Assuming $\mathrm{v}_{s}$ is for uncoupled gravitons equal (or close) to standard speed of light (c), coupling with standard matter will decrease it but also impart momentum on coupled matter.

Note that such gravitons can't accelerate matter beyond c.

At full capacity, $\mathrm{m}_{s}=\mathrm{m}, \mathrm{v}_{s}=\mathrm{v}$ and velocity becomes Keplerian:

$$
v^{2}=\frac{G M}{r}
$$

For $\mathrm{m}<\mathrm{m}_{s}$ velocity increases, at $\mathrm{m} \| \mathrm{m}_{s}, \mathrm{v} \approx \mathrm{v}_{s} \approx \mathrm{c}$. With $\mathrm{m}$ equal to the mass of a single graviton, $\mathrm{v}$ becomes equal to $\mathrm{c}$.

For $\mathrm{m}>\mathrm{m}_{s}$ velocity becomes lower than Keplerian, acting forces are no longer in balance (vacuum pressure prevails), inertia is disturbed and orbital radius decreases - all the way to relative 0 if everything below the original orbit is at full capacity (and if not stopped by accumulated orbiting mass - ie. satellites falling back to Earth are stopped at surface radius).

With such mechanism, it should be common for inner orbits to be at full capacity (or over-capacitated near the centre), with probability for under-capacitation increasing with orbital distance.

Energy accumulating in the centre will, however, generally be radiated or expelled outwards in some form through various mechanisms.

Note that $\mathrm{v}$ and $\mathrm{v}_{s}$ are generally vectors, while here it is assumed that angle between $\mathrm{v}$ and $\mathrm{v}_{s}$ is 0 . This should be fulfilled in equilibrium but might not be fulfilled otherwise and should be taken into account. 


\subsubsection{Proper relativistic treatment}

The constant c can be interpreted as the constant of proportionality between space and time. Since time dimension is a subspace of space in CR, it is relatively non-dimensional and may be treated invariant in local reference frames. However, in reality space and time dimensions can and will scale differently and the assumption of absolute invariance of c (absolute entanglement of space and time) will lead to misinterpretation (illusion).

The difference (oscillation of the constant) may certainly be negligible in horizontal energy levels and vertical sublevels, however, between major energy levels (ie. standard electron/Neptune) significant difference will exist.

Generally, c should be inversely proportional to scale.

This, of course, applies to all other dimensional constants too.

\subsubsection{Mechanism of exchange}

Exchange of electro-magnetic potential for gravitational potential is done through the change of scale. In example, radii of charge maxima may be deflated with inflation of a mass radius of a gravitational maximum. This doesn't affect only orbital radii of gravitons but also spin radii of graviton quanta - effectively, charge is subdued with the inflation of mass (gravity).

Note that, seemingly, no extra energy is required as this is simply a change of force flavour, not strength.

However, energy is needed to stimulate and process transformation, during which some energy may also be lost. The end product will then have different energy than initial total energy.

The triggers of exchange may be:

- annihilation of matter with anti-matter,

- critical temperature/density (extremely low, extremely high).

If transformation mechanism exists locally, external energy needed to stimulate exchange can be extremely low. This may simply be resonance or entanglement inversion.

Note that any entanglement decrease must be coupled with another entanglement increase.

The exchange of potentials of general force could thus be common in birth and death of bosons and boson (Bose-Einstein) condensates.

The equivalence of bosenovas and supernovas/novas, galaxies and quantum vortices, planetary systems and atoms, in that case, may go far beyond abstract similarity. 
In any case, it should not be impossible, in any universe, to ensure conditions that would reduce the relativity of self-similarity (similarity to a universe of adjacent vertical scale) to a minimum.

Ten condensed atoms of ${ }^{10} \mathrm{C}$ may just form a $\mathrm{U}_{0}$. Solar System at some moment of oscillation.

\subsection{Evaluation of $\mathrm{G}$}

Gravitational constant $(\mathrm{G})$ is not fundamental and may be interpreted as:

$$
G=\frac{1}{2} \frac{A_{s}}{M} \frac{v_{s}}{T_{s}}=2 \pi \frac{R^{2}}{M} \frac{v_{s}}{T_{s}}=\frac{3 \pi}{\rho} \frac{1}{T_{s}^{2}}=\frac{R}{M} v_{s}^{2}\left[\frac{m^{3}}{k g s^{2}}\right]
$$

$\mathrm{A}_{s}=$ surface area of the gravitational maximum [graviton] $\mathrm{R}=$ radius of the maximum $\mathrm{M}=$ gravitational mass of the maximum $\rho=$ mass density of the maximum $\mathrm{v}_{s}=$ angular velocity of the maximum $\mathrm{T}_{s}=$ period of rotation of the maximum

It is then relative to a particular graviton (gravitational maximum) and has its properties, such as mass, radius and velocity, built in. These are generally variable properties.

Even if, generally, all these variables are correlated in such a way that G remains constant, are they correlated (entangled) at all times and do changes propagate instantly?

In CR, instant propagation of information is [absolutely] impossible and some phase difference between changes in $[G$ and $]$ the variables will always exist. The $\mathrm{G}$ itself must oscillate.

Obviously, a gravitational maximum has a [changeable] spin momentum and this can further be complicated when it is evidently composed of multiple maxima.

Note that $\mathrm{v}_{s}$ here is Keplerian velocity. Assuming $\mathrm{M}$ is the mass of a graviton, non-Keplerian velocity may be interpreted as a difference in $\mathrm{G}$ (due to a difference in graviton mass).

While the 3-dimensional (spherical) form of one maximum may cloud the existence of inner maxima, outer maxima can have different spin momenta. Even if the whole system changes spin, changes cannot be instantaneous across all maxima, rather propagate in a wave-like nature. 


\subsubsection{Gravitational collapse ( $\mathrm{G}$ collapse)}

Rotational profiles of galaxies show that $1 / \mathrm{R}$ is often not proportional to $\mathrm{v}_{s}{ }^{2}$. Even if outer maxima have collapsed (fragmented) to multiple satellite maxima of smaller scale, these cannot acquire [real] mass instantaneously nor they will always acquire [real] mass during collapse.

Although collapse requires energy, it doesn't necessarily have to come from real mass. Unlike in GR, gravitational collapse in CR is not reserved for massive bodies, the energy for collapse depends on the initial energy of the maximum.

Maxima can thus remain naked for relatively long times, proportionally to scale and inversely proportionally to mass (energy) field density, before [another] equilibrium is established and $1 / \mathrm{R}$ becomes proportional to $\mathrm{v}_{s}{ }^{2}$.

Gravitational collapse in CR is localization of graviton momentum - ie. a large scale graviton with a spin radius on the order of $10^{6} \mathrm{~km}$ can collapse to form a graviton with a spin radius on the order of $10^{3} \mathrm{~km}$, sometimes with a former spin radius fossilized into orbital radius of a new body.

Real mass required for equilibrium can be obtained through conservation of angular momenta:

$$
\begin{aligned}
& {\left[m_{i m g}(n)+m_{r e}(n)\right] v_{n} r_{n}=\left[m_{i m g}(n-1)+m_{r e}(n-1)\right] v_{n-1} r_{n-1}} \\
& =M v_{s} R \\
& \mathrm{~m}_{i m g}=\text { mass of the naked gravitational maximum }=\text { imaginary mass } \\
& \mathrm{v}=\text { orbital velocity of the maximum } \\
& \mathrm{r}=\text { radius of the maximum } \\
& \mathrm{m}_{r e}=\text { acquired smaller scale mass }=\text { real mass } \\
& \mathrm{v}_{s}=\mathrm{v}_{n-1}=\text { spin velocity of the [collapsed] maximum } \\
& \mathrm{R}=\mathrm{r}_{n-1}=\text { radius of the [collapsed] maximum } \\
& \mathrm{M}=\mathrm{M}_{n-1}=\text { total mass of the [collapsed] maximum }
\end{aligned}
$$

where $\mathrm{n}$ is the scale of the maximum. In the above, dependence on scale has two equivalent notations, ie.:

$$
m_{i m g}(n)=m_{i m g_{n}}
$$

Collapse of a graviton to smaller scale generally can be a high energy or low energy collapse. In case of high energy collapse, accumulated real mass is lost (ie. nova explosions) - decoupled from a collapsing graviton. In case of low energy collapse, the graviton is [relatively] naked and collapse occurs due to localized disturbance of its momentum with sufficient real mass for coupling on smaller scale.

Note that graviton may, instead of deflation, start inflating after decoupling. It will then continue inflating until it reaches maximum range if it does not collapse and couple to another mass before that point is reached. 
Maximum range becomes a relative term if graviton is losing energy with inflation - in that case, range would be expanding continuously. However, most energy is lost with coupling.

Regardless of interpretation, lost energy at the moment of coupling should be proportional to distance between inflation/emission (decoupling) and absorption/deflation (coupling) due to increasing distance in correlation between entangled constituent quanta of the wave (graviton) - the volume of entanglement cannot remain absolutely constant and it is unlikely to conserve constancy (oscillation average) with distance.

Note also that collapse of a wave-form is only a relative collapse of wave nature - difference between a particle and a wave is simply in a radius of a wavelike form. The inverse interpretation is also valid - in some reference frames, the larger wave-form may be interpreted as a particle.

In reference frames where collapse is discrete, low energy collapse occurs when this is established:

$$
m_{\text {img }}(n)=m_{r e}(n-1)
$$

In other reference frames, collapse proceeds gradually and can start even before the above relation is established.

For a naked maximum, $\mathrm{m}_{r e}(\mathrm{n})$ is a relative 0 . In high energy collapse, $\mathrm{m}_{i m g}(\mathrm{n})$ must be decreased to match $\mathrm{m}_{r e}(\mathrm{n}-1)$. This is synchronized with increase in $\mathrm{v}_{n} \mathrm{r}_{n}$ product.

There are no fundamental constants. All are fundamentally variable.

\subsection{Reduction}

The story of forces should probably begin with a single dimension that represents a physical manifestation of correlation (entanglement) between two particles. Attractive force is then a synonym for decreasing distance in correlation, repulsive force - vice versa. However, if wave interpretation prevails, any apparent force may be interpreted as curvature of that dimension.

Assuming this is not the only such pair in existence, through interactions with other particles, distance will be randomly increasing or decreasing between pairs. This could then be interpreted as Brownian thermal force. Whether the motion/energy is the result of force (or curvature) or the other way around is completely relative.

Now assume distance in correlation in a pair reduces to relative 0 , forming a relative superposition. Assuming this entanglement is interpreted as space, the inverse of that entanglement is time. Now that entanglement will be quantized corresponding to number of all other particles in existence. If superposition is oscillating between pairs, this can locally be interpreted as oscillation of mass. 
However, that superposition is unstable due to thermal motion. And this is where the story would end if the distance would not be relative and if all this wouldn't exist at different scales.

Suppose the entanglement between vertical scales is stronger due to stronger anti-alignment in properties. Now the superposition will be harder to break with thermal motion if there is no such motion on the other scale.

With that, it becomes clear why the standard strong force is relatively unbreakable and why stable [systems of] quanta of energy have to be inverted between adjacent vertical scales.

Inversion here is commonly represented by inversion of appropriately scaled pressure and temperature.

Assume now that instead of fixed superposition, mass oscillation is fixed to certain frequencies. Obviously, this gives rise to charge - repulsive or attractive force between energies depending on correlation in frequency. Things get increasingly complicated with the number of discrete masses in oscillation.

Opposite (anti-aligned) charges are decreasing spatial distance due to increasing correlation manifested in sharing of coupled carrier particles (ie. half-photons) between them. Coupling of these particles increases carrier mass, decreasing range. This contraction of a radius of one dimension (ie. time, or magnetic field tube) is then synchronized with contraction of spatial distance. Decrease of distance will be accelerated due to positive feedback (the rate of fusion of carriers increases inversely proportionally to distance).

The [superposition of] carrier particles is thus alternating in entanglement between two sources of force (synchronized with mass oscillation). If charges (frequencies of mass oscillation) are aligned there can be no sharing and carriers will be annihilating or deflating into smaller scale, increasing range.

Note that difference in velocities between two charges can affect mass oscillation and, therefore, alignment of frequencies, when relativistic effects are manifested locally.

This implies that rates of nuclear fusion should increase if fusing protons have different velocities.

One interpretation of strong force could then be oscillation of particles between $2^{*} 3$ different mass (colour charge) oscillations, giving $2^{3}$ combinations for gluons (coupled carriers). 


\section{Scale and centre of the observable universe}

For every universe there must exist a reference frame that universe is revolving about.

Given the determined scales of discrete vertical energy levels, it is reasonable to assume that the centre of the observable universe (point it is revolving about) is outside of it.

Thus, all observable galaxies orbit a barycentre outside of the observable universe. These can be confirmed and approximated by observing galaxies on a different energy level of the same system.

Momenta of such galaxies should be correlated - in equilibrium state they orbit the centre in the same direction but with a calculable difference in speed and distance from the centre.

Ie. If one considers Milky way as a bound electron, the atom which Milky way is a part of should have a diameter on the order of 10 trillion $\left(10^{13}\right)$ light years, $10^{3}$ times the diameter of the observable universe. In that case, all other energy levels are outside of the observable universe.

However, considering the number of galaxies and the state of evolution, this is not the best interpretation (although some layering may be present).

Considering distances between atoms (planetary systems) and molecules (binaries and other strongly correlated systems) the observable universe is a gas bubble of extremely low density with particles concentrated in quantum vortices (galaxies).

This bubble or soup, however, cannot be completely homogeneous and it is only a matter of technology and proper interpretation whether one can observe the difference between the closest and the furthest layers of this gas (layer) relative to the external central point.

The most appropriate interpretation of observable universe is a part of space of a large scale graviton, in a form of a torus - as stated already.

It may have been deflated from an even larger scale, however, evidence suggests it has been inflated from smaller scale, probably in an annihilation event.

\section{Atomic property differentiation between sys- tems}

Vertical energy levels are entangled. This implies entanglement between equal species, but entanglement between different species of different scales is not forbidden either.

Increasing number of protons and electrons in an atom is splitting (or increasing) energy levels (layers) of the atom.

What if properties of standard scale atoms are strongly correlated with properties of $\mathrm{U}_{1} / \mathrm{U}_{-1}$ systems (atoms) they are co-evolving with?

For example, radii of atoms might be correlated with [a density of] the gravitational well they are in. 
Consider the Lyman (or any other) series for a hydrogen [like] atom - if density of series is not invariant to such correlation, one could have a distorted image of non-local reality, as the spectrum lines of standard atomic elements would be variable across time and planetary systems.

Ie. in the Nitrogen system the Lyman series for hydrogen would have redshifted frequencies.

One might even argue there are 6 distinct wavelengths (after 6th, the spectrum becomes continuous) in Lyman series and that such differentiation is a direct consequence of the Solar System being an atom with 6 protons and 6 electrons (carbon), or that series beyond the 6th may be influenced by more distant systems.

\section{Wave-particle duality}

All particles having a momentum always generate waves. A particle itself may be in wavelike or corpuscular form. This form, like everything, is relative. One observer might detect a wave while other may observe a particle form (both forms can even be observed at the same time). Forms are dependent on properties of local space, which may be affected by the observer too.

Generally, with more energy density, a corpuscular form is more likely to be detected. These are coherent waves which have more concentrated mass due to wave collapses initiated by self interference. Waves may be coherent in space (laser waves) and/or in time (high frequency waves). Coherence in both, space and time, will thus produce the most dense energy.

A wave collapse may also be initiated by interaction with other waves (particles).

From human perspective, wave nature prevails on $\mathrm{U}_{-1}$ scale, particle/wave on $\mathrm{U}_{0}$, while on $\mathrm{U}_{1}$ scale nature is observed generally as corpuscular.

New particles can be formed with two species of a single particle/wave (ie. half-photon) in coherence, fused (concentrated, with range decrease) to form a particle of larger scale (mass).

\section{Evidence}

Some evidence for complete relativity is presented in this paper. Additional evidence confirming complete relativity of universes is presented in follow-up papers. Mainly in the analysis of the Solar System in CR context[8].

Of course, other researchers are welcome to confirm or refute the presented predictions and hypotheses in this and follow-up papers.

Due to inherent limits in observation, however, different interpretations are sometimes possible and some might prefer a less-intuitive interpretation of real- 
ity. This does not have to be an issue - there's no reason we should all believe in the same reality, especially that which we cannot observe directly. Some views, however, may be limiting the observer of that reality.

\section{Conclusion}

Angular momentum seems to form the fundamental signature of energy. All energy is thus fundamentally correlated with frequency. Evolution might concentrate these energies into corpuscular forms of different scale, however, oscillation will always be relatively conserved and observable at all scales of space taking dilated time into account. But the single absolutely intrinsic property of a universe is relativity.

It appears that everything must be relative in order to exist, and conservation of energy through transformation (evolution) is inevitably equivalent to conservation of relativity.

\section{Acknowledgement}

This would not be possible without all the work of all the observers of observables of different scale, theorists describing physics of such universes and promoters and propagators of knowledge.

This would also not be possible without Earth providing souls and energy for all these creatures, without the Sun providing soul and energy for Earth and without the galaxy providing soul and energy to Sun. ...

Last, but not least, this would not be possible without the universes of my body entangled with my soul, often enduring sleepless nights with understanding rather than rebellion.

Thus, I thank everything for every thing and every thing for everything.

\section{References}

[1] Fast galaxy bars continue to challenge standard cosmology (2021), M. Roshan et al, Mon. Not. R. Astron. Soc. 508(1), 926-939 https://doi.org/10.1093/mnras/stab2553

[2] From Galactic Bars to the Hubble Tension: Weighing Up the Astrophysical Evidence for Milgromian Gravity (2022), I. Banik and H. Zhao, Symmetry $14(7), 1331$ https://doi.org/10.3390/sym14071331

[3] Putovanje kroz vrijeme (2021), Amenoum, Universum Amenoum: D. Log https://amenoum.org/log/31_putovanje_kroz_vrijeme.html

[4] What is a Spinor? (2021), M. Hughes https://in-theory.net/what-is-a-spinor/ 
[5] The variance of the CMB temperature gradient: a new signature of a multiply connected Universe (2021), R. Aurich et al, Classical. Quant. Grav. $38(22), 225005$

https://doi.org/10.1088/1361-6382/ac27f0

[6] The Universe Is a Giant Donut That We Live Inside, New Research Suggests (2021), S. Wells https://www.vice.com/en/article/3aqjkn/the-universe-is-a-giant-donutthat-we-live-inside-new-research-suggests

[7] The power of relativistic jets is larger than the luminosity of their accretion disks (2014), G. Ghisellini et al, Nature 515, 376-378

https://doi.org/10.1038/nature13856

[8] The Solar System: Nature and mechanics (2021), Amenoum, Universum Amenoum https://amenoum.org/solar_system.html

[9] A general argument against superluminal transmission through the quantum mechanical measurement process (1980), G. C. Ghirardi et al, Lett. Nuovo Cimento 27, 293-298 https://doi.org/10.1007/BF02817189

[10] Precise Charm to Strange Mass Ratio and Light Quark Masses from Full Lattice QCD (2010), C. T. H. Davies et al, Phys. Rev. Lett. 104, 132003 https://doi.org/10.1103/PhysRevLett.104.132003

[11] Evidence for intrinsic charm quarks in the proton (2022), The NNPDF Collaboration, Nature 608, 483-487 https://doi.org/10.1038/s41586-022-04998-2

[12] Mass radius of the proton (2021), D. E. Kharzeev, Phys. Rev. D 104, 054015 https://doi.org/10.1103/PhysRevD.104.054015

[13] Planetary Fact Sheets (2021), NASA https://nssdc.gsfc.nasa.gov/planetary/planetfact.html

[14] Five Years after New Horizons' Historic Flyby, Here Are 10 Cool Things We Learned About Pluto (2020), NASA https://www.nasa.gov/feature/five-years-after-new-horizons-historicflyby-here-are-10-cool-things-we-learned-about-plut-0

[15] Craters of the Pluto-Charon system (2017), S. J. Robbins et al, Icarus 287, 187-206 https://doi.org/10.1016/j.icarus.2016.09.027

[16] Impact craters on Pluto and Charon indicate a deficit of small Kuiper belt objects (2019), K. N. Singer et al, Science 363(6430), 955-959 https://doi.org/10.1126/science.aap8628 
[17] A new volcanic province: an inventory of subglacial volcanoes in West Antarctica (2018), M. V. W. De Vries et al, Geol. Soc. Spec. Publ. 461(1), 231-248

https://doi.org/10.1144/SP461.7

[18] Mercury's Interior Structure Constrained by Density and P-Wave Velocity Measurements of Liquid Fe-Si-C Alloys (2020), J. S. Knibbe et al, JGR Planets 126(1), e2020JE006651 https://doi.org/10.1029/2020JE006651

[19] The Internal Structure of Mercury's Core Inferred From Magnetic Observations (2021), I. Wardinski et al, JGR Planets 126(12), e2020JE006792 https://doi.org/10.1029/2020JE006792

[20] Tidal constraints on the interior of Venus (2017), C. Dumoulin et al, JGR Planets 122(6), 1338-1352 https://doi.org/10.1002/2016JE005249

[21] Mars's core has been measured - and it's surprisingly large (2021), A. Witze, Nature 591, 514-515 https://doi.org/10.1038/d41586-021-00696-7

[22] Characteristics of memories for near-death experiences (2017), L. E. Moore and B. Greyson, Conscious Cogn. 51, 116-124 https://doi.org/10.1016/j.concog.2017.03.003

[23] Correlation of the highest-energy cosmic rays with the positions of nearby active galactic nuclei (2008), J. Abraham et al, Astropart. Phys. 29(3), 188-204

https://doi.org/10.1016/j.astropartphys.2008.01.002

[24] Indications Of Intermediate-Scale Anisotropy Of Cosmic Rays With Energy Greater Than $57 \mathrm{EeV}$ In The Northern Sky Measured With The Surface Detector Of The Telescope Array Experiment (2014), R. U. Abbasi et al, Astrophys. J. Lett. 790(2) https://doi.org/10.1088/2041-8205/790/2/L21

[25] First Observation of the Greisen-Zatsepin-Kuzmin Suppression (2008), R. U. Abbasi et al, Phys. Rev. Lett. 100, 101101 https://doi.org/10.1103/PhysRevLett.100.101101

[26] Cosmology from quantum potential (2015), A. F. Ali and S. Das, Phys. Lett. B 741, 276-279 https://doi.org/10.1016/j.physletb.2014.12.057

[27] The empirical case for 10-GeV dark matter (2012), D. Hooper, Phys. Dark Universe 1(1-2), 1-23 https://doi.org/10.1016/j.dark.2012.07.001 
[28] Rest mass of photon on the surface of matter (2020), M. Goray et al, Results Phys. 16, 102866

https://doi.org/10.1016/j.rinp.2019.102866

[29] Solution to gravitational anomalies: Reincarnation and karma of consciousness (2022), Amenoum, Universum Amenoum: D. Log

https://amenoum.org/log/7_solution_to_gravitational_anomalies.html

[30] Indication, from Pioneer 10/11, Galileo, and Ulysses Data, of an Apparent Anomalous, Weak, Long-Range Acceleration (1998), J. D. Anderson et al, Phys. Rev. Lett. 81, 2858

https://doi.org/10.1103/PhysRevLett.81.2858

[31] When leaving the Solar system: Dark matter makes a difference (2022), E. Belbruno and J. Green, Mon. Not. R. Astron. Soc. 510(4), 5154-5163 https://doi.org/10.1093/mnras/stab3781 